\title{
A METHOD FOR COMPUTER SIMULATION OF PROBLEMS IN SOLID MECHANICS AND GAS DYNAMICS IN THREE DIMENSIONS AND TIME
}

\author{
M. L. Wilkins \\ -R. E. Blum \\ E. Cronshagen \\ P. Grantham
}

April 24,1974

Prepand for US Atomic Energy Commission under contract No.W-7405-Eng-48

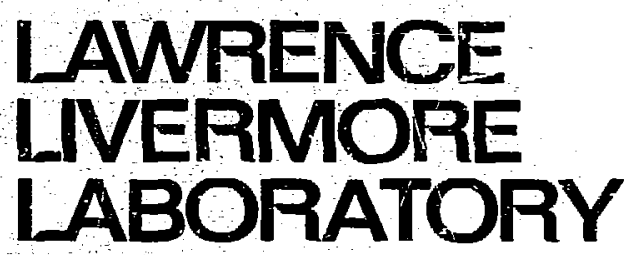

University of Califomia/Livermore 


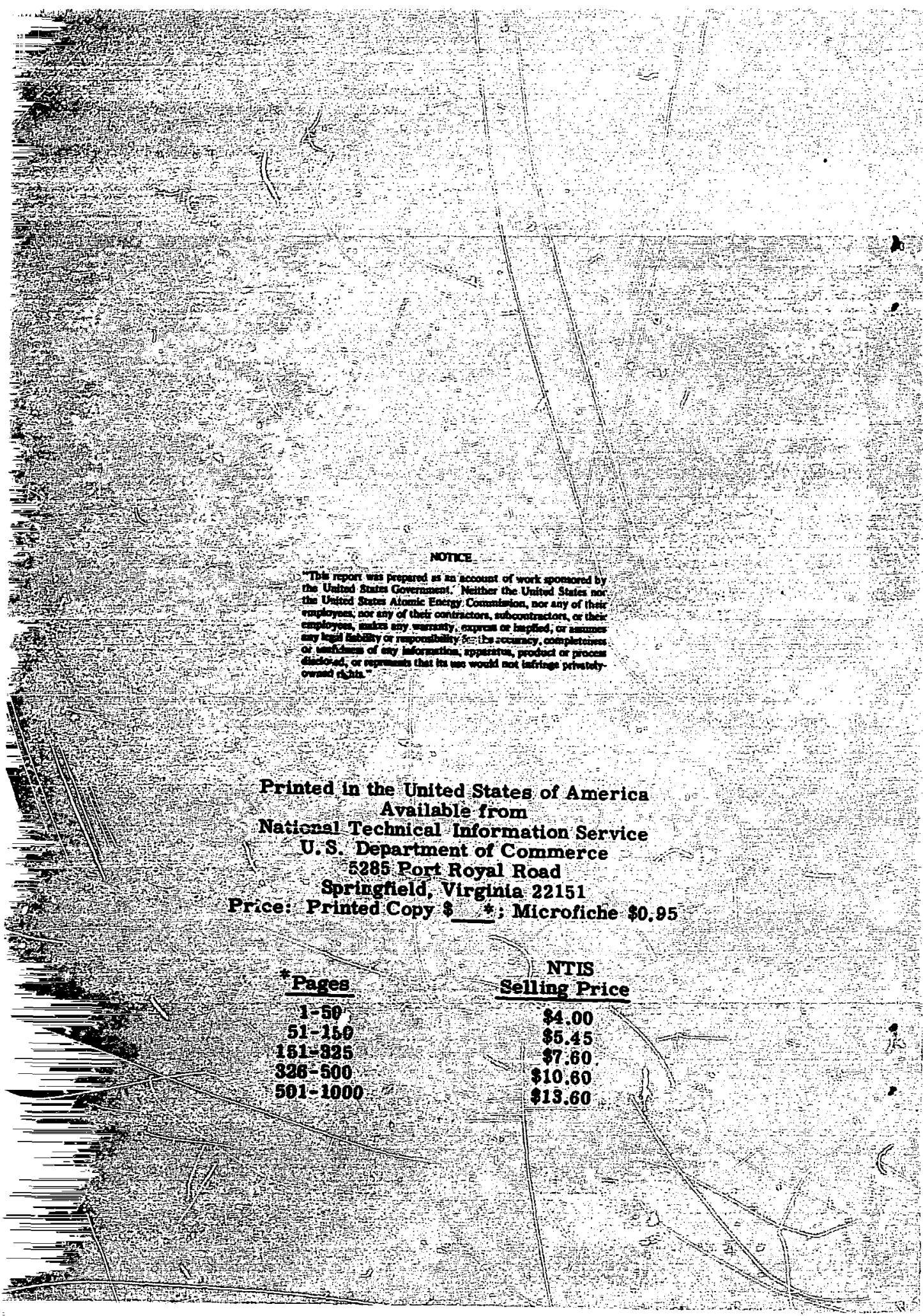


TID-4500, UC-32

Mathematics and Computers

\section{농 \\ LAWPENCE LVEFMORE LABOPATOFY \\ Uriersity of Cafiomia/Livemora, Caffornia/94550}

\section{UCRL-51574 \\ A AETHOD FOR COMPUTER SIMULATION OF PROBLEMS IN SOUD MECHANICS AND GAS DYNAMICS IN THREE DIMENSIONS AND TIME}
M. L. Wilkins
R. E. Blum
E. Cronshagen
P. Grantham

MS date: April 24, 1974

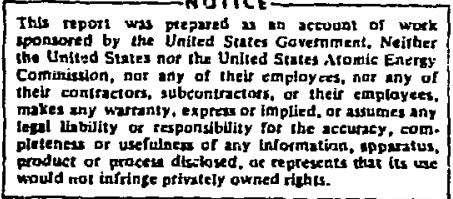

Itis report was piepared 21 in actoont of wotk

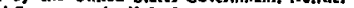
Coministan, nat any of thete toptorces, nor any of theit conifactors, subeuniractory, or their emplayes. legal lability os respondibitity rot the aceuras com. piateness or usefulacen of any tniormatipa, pposatus. would mol infringe ptivalely owned rights. 


\section{Foreword}

This work was supported by the Office of Materials Science, Defense Advanced Research Projects Agency, under the auspices of the United States Atomic Energy Commission. 


\title{
Contents
}

\begin{abstract}
. . . . . . . . . . . . . . . . . . 1

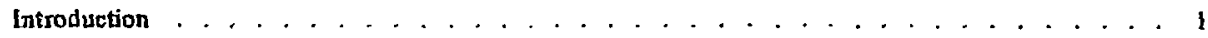

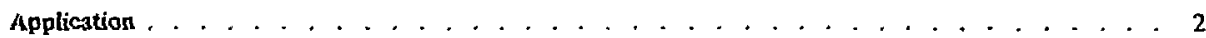

Summary and Conclusions . . . . . . . . . . . . . . . . . . . . 3

Appendix A: The SAP Coife for Static Three Dimensional Finite Element Stress Analysis. . . . . . . . 8

Appendix B: HEMP 3D, a Finite Difference Code for Calculating Elastic-Plastic Flow . . . . . . . . . 13

Appendix C: Comparison of the Finite Difference and Finite Element Methods for the Solution of Poisson's Equations. . . . . . . . . . . . . . . . . . . 37

Appendix D: HEAT 3D, a Code for Calculating Thermal Diffusion in Three Dimensions. . . . . . . . . 49

Appenuix E: VMESH 3D, a Zoner Program for Semisutomatic Generation of

Three-Dimensional Meshes. . . . . . . . . . . . . . . . . 60 60

Appendix F: VPIX 3D, a Graphic Program for Interactive Display of Three-

Dimensional Objects. . . . . . . . . . . . . . . . 71

References . . . . . . . . . . . . . . . . . . . . . 82
\end{abstract}




\title{
A METHOD FOR COMPUTER SIMULATION OF PROBLEMS IN SOLID MECHANICS AND GAS DYNAMICS IN THREE DIMENSIONS AND TIME
}

\begin{abstract}
Details are given for a versatile computer program that employs finite element and finite difference techniques to describe complex physical phenomena in three dimensions and time. Applications include problems in gas dynamics, elasticity, dynamic plasticity, fracture, and thermal conduction. The main features of the program are illustrated by a calculation that deternines the thermal-shock stresses in a stator blade of a gas turbine engine. Appendices present an outlire of SOLID SAP, a conplete mathematical development of HEMP 3D, a comparisor. between finite difference and finite element methods, and descriptions of subroutines for discretizing, for display, and for calculation of three-dimensional thermal diffusion.
\end{abstract}

\section{Introduction}

Continuum mechanics provides a very effective approach to the study of cumple $\hat{A}_{i}$ physical plenomena, ranging from detonation theory and gas dynamics to the failure of solids by elastic-plastic flow and subsequert fracture. The general mathematical problem is the requirement for solving, in three space dimensions and time, the three fundamental equations of physics: conservaticn of mass, momentum, and energy, coupled with a set of equations that descrite the physical behavior of the material. Material behavior represints the major unknown.

Materials whose behavior over some limited range can be described by a perfect gas equation-of-state, or by Hcoke's Law, represent simple extremes. The description of the behavior ef a given material over a large range of temperature and pressure, for example, is considerably more complicated. In the case of the engineering application of solids, the material description involves a stress tensor, failure criteria for plastic flow and fracture, and possible temperature and time effects.

The structural analyst faces an enormous problem in the solution of the three fundamental equations if the physical object has a complicated geometry. Even for relatively simple geometries, large, high-speed computers are the only option. Computations in three space dimensions and time taxes the capacity of even the largest computers.

In the development of a computing procedure, there are three basic tasks:

- Generate a calculational grid that characterizes the physical object. 1 inis process-called discretization-is an extremely complex problem in three dimensions.

- Incorporate a numerical scheme for solving the appropriate partial dilferential equations that is accuratz, efficient and versatile for applying boundary conditions.

- Develop computer graphics capable of noonitoring the computer input and displaying the computer output. This requirement is especially critical in a tluree-dimensicnal analusis, due to large collection of numbers involved.

The computer progiam report sd !er:, icvelcped for the LLL STAR and 7600 computers, includes the above three features.

Vector mode programming has been employed for the most efficient use of the computers. The numerical methods utilize implicit finite element (FE) and explicit finite differences (FD) integration schemes. The FD aspect of the program, HEMP 3D, is a three dimensional analog of the two dinensional HEMP ${ }^{1}$ code for cale dating elastic-plastic flow. HEnIP $3 \mathrm{E}$ is formulated in Lagrange coordinates in three dimensicns and time. Arbitrary 
models of material behavior may be used including nonlinear work hardening. time dependent phenomena and ductile and brittle fracture. The FE compuier code, SOLID SAP? was obtained from Prof. E. Wilson, University of California. SOLID SAP is well suited for obtainis: static solutions of problems in solid mechanics where linear elasticity theory of material behavior can be applied.

A grid generator has been developed that permits applying the same calculational grid to botl SOLID SAP and HEMP 3D. Information can be transferred from one ccle to the other. and the three-dimensional (3-D) plot routines apply to both codes. The ablity to start a calculation on thi dynamic HEMP 3D code using initial conditions from the static SOLID SAP code can provide an importani saving in computer time.

The objective here is to minimize computation time by being able to apply both FE and FD numerical methods. It is obviously important for calculations in three space dimensions to have the fastest program available.

The programs can also be used in two space dimensions. In this case it is more economical in computer tinue to couple the FE code with the two-dimensional (2-D) HEMP code. ${ }^{1}$

The thermal diffusion equation

$$
\begin{aligned}
& \frac{\partial T}{\partial t}=k \nabla^{2} T \quad T=\text { temperature } \\
& t=\text { time } \\
& k=\text { coefficient of thermal diffusion }
\end{aligned}
$$

can be incorporated in either SOLID SAP or HEMP 3D and is solved in three space dimensions and tine by an implicit numerical scheme. The accuracy of the numerical method is of the order of $0.1 \%$ when compared to selected problems where a solution can be obtained by other means.

The overall program can be used to simulate, in three space dimen.ions and time, physical phenomena that include

gas dynamics,

elasticity theory,

dynamic plasticity,

fracture, and

thermal conduction.

\section{Application}

An example that exercises some of the features of the program is provided by the analysis of thermal stresses in a stator blade of a gas turbine. The specific problem chosen is to calculate the stresses induced in the first stage stator at startup when hot gas suddenly strikes the concave surface of the airfoil. The material is assumed to

\begin{tabular}{|c|c|c|}
\hline & Sl units & Customary units \\
\hline Shear modulus & $83 \mathrm{GPa}$ & $\left(12 \times 10^{6} \mathrm{psi}\right)$ \\
\hline Bulk modulus & $140 \mathrm{GPa}$ & $\left(20 \times 10^{6} \mathrm{psi}\right)$ \\
\hline Density & $2.24 \mathrm{Mg} / \mathrm{m}^{3}$ & $\left(0.081 \mathrm{lb} /\right.$ in. $\left.^{3}\right)$ \\
\hline Thermal conductivity & $16.7 \mathrm{~W} / \mathrm{m} \cdot \mathrm{K}$ & $\left(2.23 \times 10^{-4}\right.$ Btu-in./in. ${ }^{2}$ sec- $\left.^{\circ} \mathrm{F}\right)$ \\
\hline Coefficient of thermal expansion & $2.75 \mu \mathrm{m} / \mathrm{m} \cdot \mathrm{K}$ & $\left(1.528 \times 10^{-6}\right.$ in./in. $\left.{ }^{\circ} \mathrm{F}\right)$ \\
\hline Specific heat & $660 \mathrm{~J} / \mathrm{kg} \cdot \mathrm{K}$ & $\left(0.16 \mathrm{Btu} / \mathrm{b}-^{\circ} \mathrm{F}\right)$ \\
\hline
\end{tabular}
be $\mathrm{Si}_{3} \mathrm{~N}_{4}$ with the material constants listed in Table $\mathrm{l}$.

Table 1. Material constants for $\mathrm{Si}_{3} \mathrm{~N}_{4}{ }^{\mathrm{a}}$.

any consistent set of units may be used. 
Working from a blueprint provide $J$ by the manufacturer, the stator blade was divided into five conceptually distinct parts: the base, the airfoil, the top plate, and the two vertical fins attached to the top plate. Equations were used to describe the surfaces of the various parts. The airfoil was discretized first by partitioning the enclosed volume into sub-regions. The ramaining components were then partitioned so that the subdivisions matched at the interfaces of the assembled components.

Figure la shows the turbine blade with the calculational grid. Figures $I b$ and $l c$ show views of the calculational model with the grid lines eliminated, and a siading routine used to evamine the surfaces. The actual stator blade appears in Fig. Id.

The boundary conditions assume a constant temperature, $T=1005 \mathrm{~K}$ applied to the concave side of the airfoil, while all other surfaces are considered insulated. The computed output provides the spatial distribution of temperature as a function of time. The two vertical fins, being relatively unimportant in this calculation, were omitted to economize computer time.

Figure 2 shows calculated temperature contours on the blade surface at time, $t=0.03 \mathrm{~s}$. The contours are obtained by linear interpolation of the temperature field. Figure 3 shows the temperature distribution in a slice parallel to the airfoil length and breadth. At this time, $t=0.03 \mathrm{~s}$, the temperature has diffused into the base plate, increasing the temperature to $T=811 \mathrm{~K}$ immediately below the heated surface. The top plate, due to the greater airfoil thickness, is essentially cold at this time.

The next step in the analysis is to compute the temperature-induced stresses. A finite element method was used to compute the steady-state stresses corresponding to the given temperature distribution. This step required the inversion of a $4000-$ by- 4000 matrix. Boundary conditions were applied corresponding to the blade assembly being rigidly mounted on the lower surface.

Figure 4 shows contours of the maximun principal stress (tensile stress) for two different stices through the blade.

\section{Summary and Conclusions}

The calculation of themally induced stresses in a turbine was presented as a demonstration of the computational program to solve problems involving 3-D geometries. The calculation assumed relatively simple thermal and mechanical boundary conditions. However, the program can incorporate temperature and mechanical boundary conditions that vary in space and time. Hooke's Law and a linear thermal stress term were used to describe the material behavior. The general program can incorporate more complex material models, including elastic-plastic flow, with nonlinear work-hardening.

Static solutions to problems in solid mechanics can be obtained using the time dependent HEMP programs by slowly applying the required boundary conditions. In two space dimensions the finite element technique used by SOLID SAP is faster than the finite difference method of the 2D HEMP program for obtaining static solutions to problems where linear elasticity theory applies. For nonlinear material behavior .the finite difference method becomes faster and is more versatile. In 3-D problems, however, the large number of zones involved made the finite difference method of the HEMP 3D program faster than SOLD SAP even for static problems. 

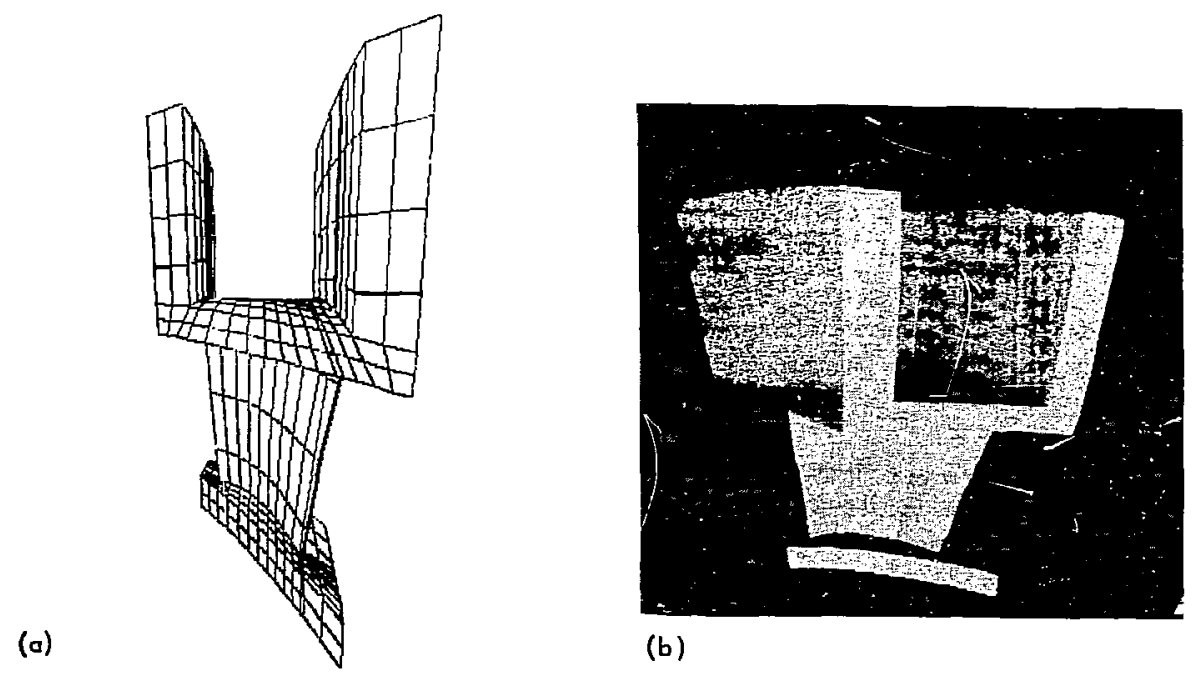

(b)
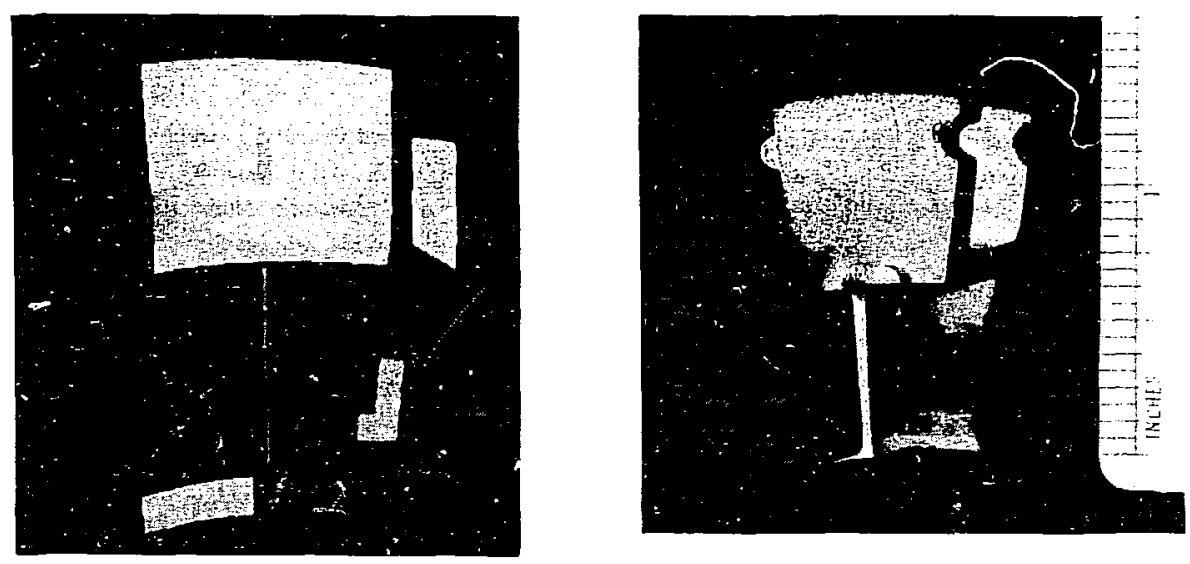

(c)

(d)

Fig. 1. Turbine stator blade geometry. (a) Calculational mesh. (b) and (c) Mesh surfaces with computer shading. (d) Actual turbine stator blade. 


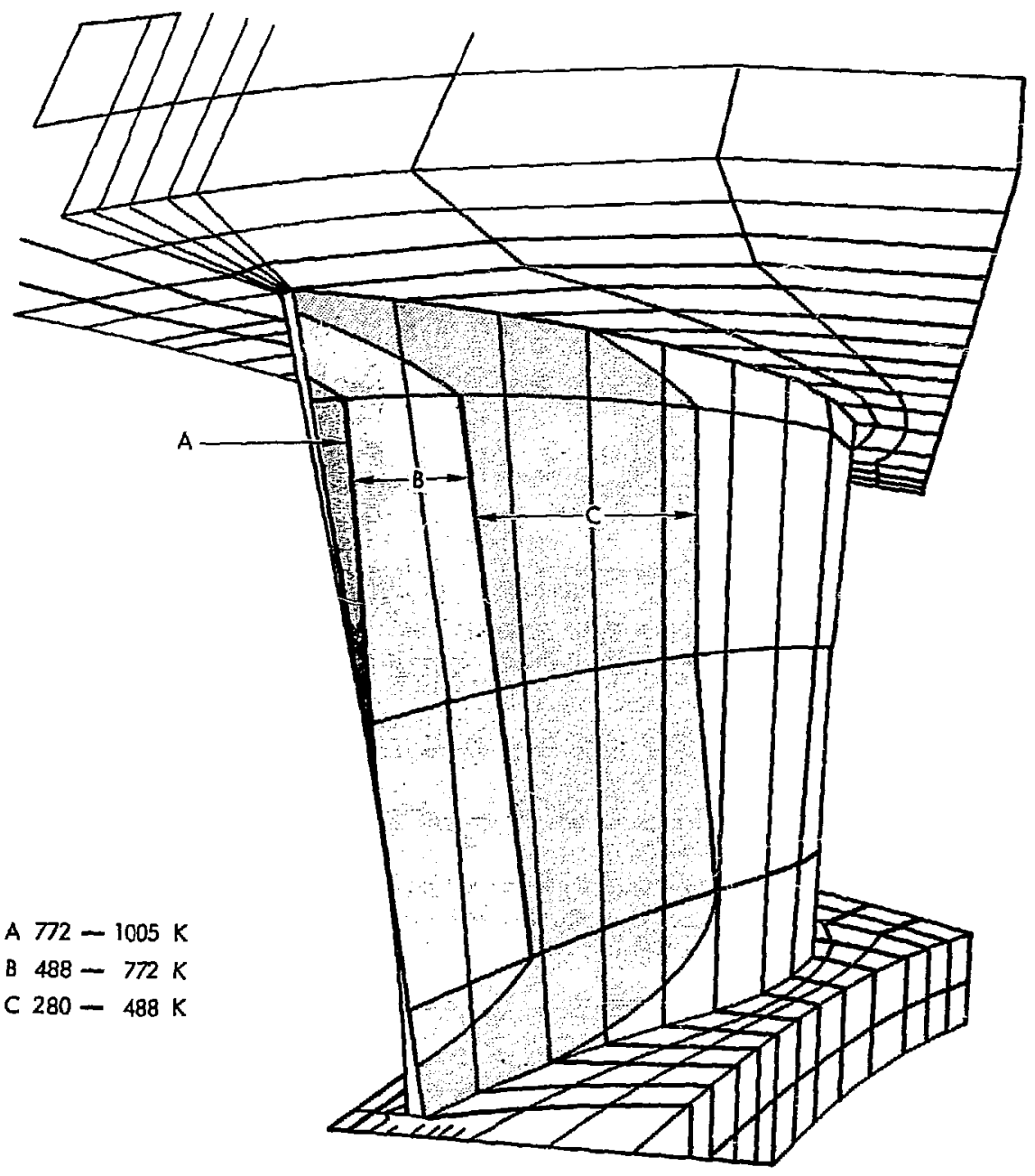

Fig. 2. Temperature profiles on the turbine stator blade surface opposite to the heated surface. 


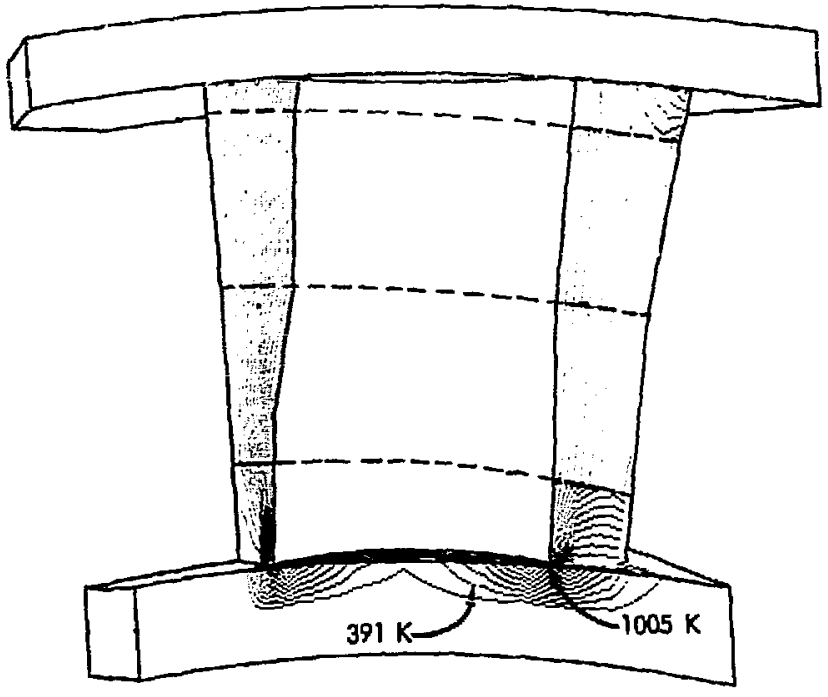

Fig. 3. Temperature distribution in a slice parallel to the st3tor blade length and breadth.

Considerable work has been done to increase the speed of the SAP program using state-of-the-art numerical schemes for resolving the large matrix that occurs in 3-D geometry with the finite element method. The HEMP 3D program was still 2-3 times faster for the same static problems. Since the HEMP 3D program can yield both static and dynamic results with arbitrary models of material behavior, future work will concentrate on this approach.

\section{ACKMOWLEDGEMENTS}

The authors extend grateful acknowledgements to Mike Archuleta for his cooperation and advice in fittung hus nislucs-line programs to our needs, to $S$. John French, Jr., for programming the initial version of the HEMP 3D code, to Alan fiisdmarsh for writing an efficient matrix inversion algorithm, and to Gary Long for providing efficient $\mathrm{I} / \mathrm{O}$ packages together with much sound general programming advice. 
(a)

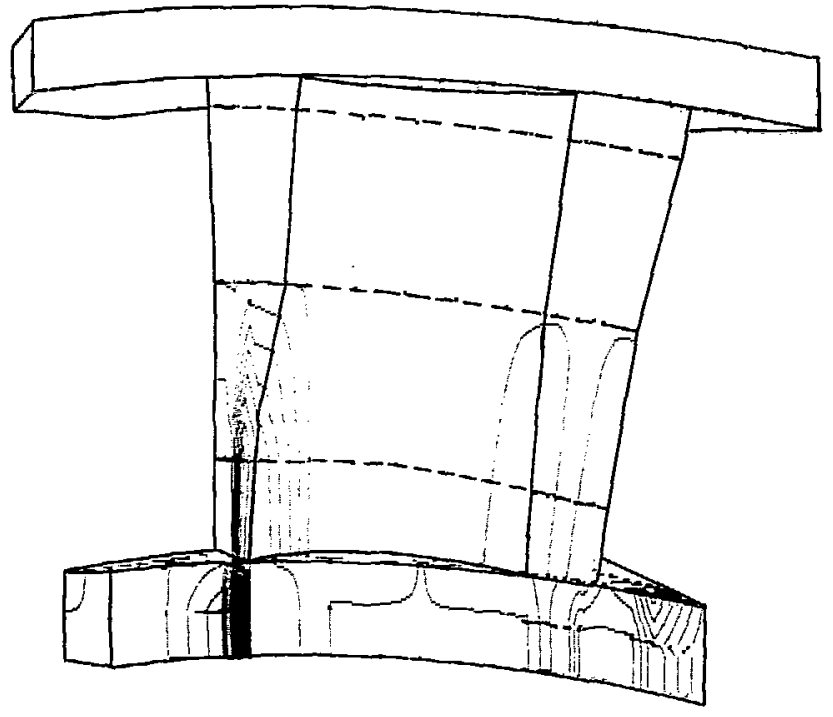

(b)

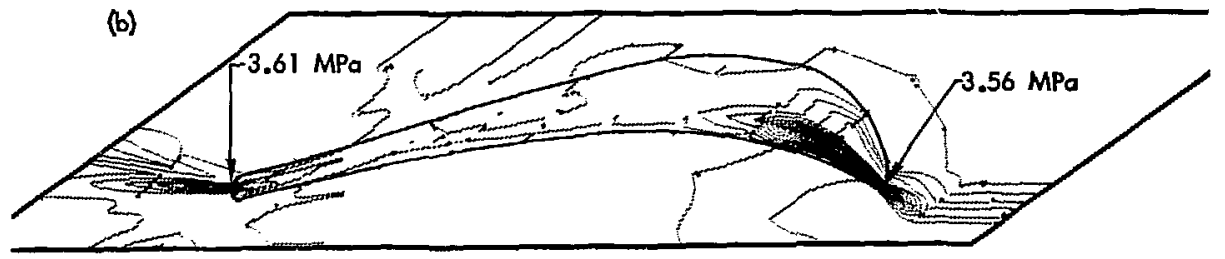

Fig. 4. Stress profiles at $t=30 \mathrm{~ms}$. (a) Maximum principal stresses in a slice parallel to the airfoil length and breadth. (b) Maximum principal stresses in a plane $2 \mathrm{~mm}$ beneath the airfoil/base interface. 
Appendix A

The SAP Code for Three Dimensional Finite Element Stress Analysis

\section{PROGRAM DESCRIPTION}

The code SAP, originated by Prof. E. Wilson, ${ }^{2}$ is a static analysis program using linear clasticity theory. The program can use general boundary conditions for solving problems in solid meclianies in two or three space dimensions. Although the mathematics of the code are unchanged. its data-basc-handling logic was extensively modified. Initialization of the loaded esde sets up common blocks for direct calls to the system for input/output (I/O) handling. In addition, three extended core memory buffers are reserved, four scratich files are created, and the input file heading is read. In production mode, the smail core memory (SCM) workspace is expanded to take in all available small core: at this point the problem will be terminated if available small core memory is insufficient. The node points coordinates and boundary condition codes are read from the disc and block copied into their workspace locations. A subroutine for numbering and storing the equations on disc, is called.

After temperatures are read from the input file, the subroutine which formis the elemental stiffness matrices is called. Included in this subroutine is the logic for element generation for blocked dats. The elemental stiffness matrix routine makes leavy use of large core memory (LCM) buffering and, with large problems, is $100 \%$ cpu efficient. The problem input is completed witen the joint loads are read in binary coded digital (BCD) form and written as the righth-hand side onto a disc file. Large blocks of elemental stiffness matrices are read, their direct sum formed, and the resultant global stiffness matrix stored on disc.

Two routines can be used to invert the stiffness matrix. Both solvers use a Gaussian elimination teclinique: one routine must be able to hold a matrix having cardinality of the bandwidth squared. and the other will take arbitrarily large bandwidth. The latter solver is essentially the solver on Wilson's origina! code; ${ }^{2}$ it pays for its generality with a heavy price in $\mathrm{Y} / \mathrm{O}$. The other solver should be used if possible. The user should be aware that there are two core size options for running with the new solver. In the lasge option. the code will attempt to use every word of the machine memory and, hence, should not be used in timesharing mods.

Finally, the displacement and stress matrices are formed from the answer obtained in the solver, and outputted both as a BCD high-speed printer file and as a binary file in a standardized form FROMSAP. For use of this binary file, the user should consult the section on postprocessors, below.

\section{SUMMARY OF THE THEORY}

The finite element snlution is, for the purposes of our discussion, a Rayleigh-Ritz approximation to the solution of a functional equation. Specifically, for a given 3-manifold, $M$, we are interested in finding a

$$
\text { uE } \mathrm{V} \subset\left\{\mathrm{v}: \mathrm{M} \rightarrow \mathbb{R}^{3}\right\}
$$

so that

$$
F[u]=\operatorname{veV}_{v \in V}^{g l b} F[v]
$$


(Physicaliy. u may be thought of as displaciment and $F[u]$ as the associated potential energy.)

If $V$ has a subsel $\left\{w_{i}\right\}$ that is complete in $V$. then we an find a seguence

$$
v_{k}(x, y, z)=\sum_{k=1}^{k} \alpha_{i} w_{i}(x, y, z)
$$

so that

$$
F\left|v_{k}\right|>F\left[v_{k+1} \mid>\lim _{i \rightarrow \infty} F\left[v_{i} \mid \geqslant F(u)\right.\right.
$$

If, in facl, F $\left|\gamma_{k}\right|$ is continsously differentiable will respect to $\alpha_{i}$ we can solve for the $\alpha_{j}$ 's directly by solving

$$
\frac{\partial F\left\lfloor v_{k} \mid\right.}{\partial a_{i}}=0 \text { for eocli } i
$$

Since the requirement that $V$ is tomplete is satisfied in most physical applications (c.a.. $V$ is frequently is Hilbert space), the onty difficulty arises in the choice of a basis set.

The finite element code, SAP, may be thought of as solving the Poisson equation

$$
k \nabla_{u}=g
$$

on the 3-manifold R. In terms of our above discussion. $F$ is the Poisson integral. The element Wilson chcse in his code is a hexahedron with nonzcro volume, where the interpolating function is given by

$$
i_{12}=\sum_{j=1}^{12} a_{i} w_{i}
$$

and where

$$
w_{i}= \begin{cases}1 \ldots \ldots i=1 & \\ \frac{1}{8}(1 \pm \xi)(1 \pm \eta)(1 \pm 5) i=2, \ldots, 9 \\ 1-\xi^{2} & i=10 \\ 1-\eta^{2} & i=11 \\ 1-5^{2} & i=12,\end{cases}
$$

$(\xi, \eta, \zeta)$ being defined on the cube of edge length, 2, and centered at the origin. The value of the element at some specified point of the manifold can be found by an appropriate translation and rotation.

In the code, the numerical procedure consists of evaluating the functional (anintegral) for each element and then forming the direc. sum over all the elenents. One obtains a matrix equation of the form

$$
\mathbf{A U}=\mathbf{G}
$$

where $A$ is colled the stiffess matrix and $G$ is the applied load. The answer then is found by evaluating

$$
U=A^{-1} \mathbf{G}
$$




\section{OPERATION}

\section{Input}

Input to the SAP code is from two files. One file is binary. called LNK. and the other is BCD. called INDAT. The binary file would be the product of the generator and should assume the standard form adopted for the three-dimensional package of codes. This form consists of a 512 -word hating and the $x$. $y$, and $z$ coordingtes, the floating point material number, and a floating point, one-or-zero nag, to denote constraint in each of the three dimensions. The heading consists of the common block described in FORTRAN as

\section{COMMON/ADDI/NUMBLK,NUMNPS,NUMNPT(10),NUMINPP(10),IPT(10).JPT(LU).KPT(10;}

The file can best be described in terms of the diagram at the right, where $n$ is the number of nodes.

The convention for the meaning of the one-zero flag is the opposite of that used internally in SAP; i.e.. a one indicates that a point is free to move while a zero means that it is constrained.

The BCD file resembles the input file of the original code closely, and users may wish to consult Ref. 2 for further details. The first card image is the label given to the problem (FORMAT 12A6); the next card is a flag, which, when set to one, terminates the problem after it is set up (FORMAT(15)). This flag is useful in dirgnosing problems in the input data. The next card lists the element number (in this version equals 5), the number of distinct materials, and the number

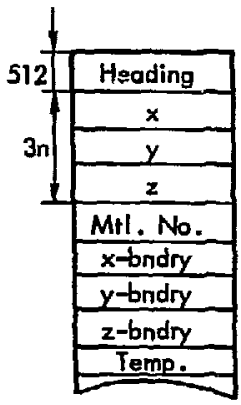
of distributed load sets (FORMAT 315). A card for each material is then needed giving a material identification number $(1<n<59$ ), the modulus of elasticity, Poisson's ratio, weight density, coefficient of thermal expansion, and yield strength (FORMAT 15, 5F 10). Loads are also given in the BCD file; for a discussion of this data, the reader is referred to pages B-26 through B-29 and B-5 of Wilson's manual. ${ }^{2}$ The formatting and conventions remain unchanged.

\section{Output}

Output from the SAP code, like the input, is in two forms: a binary file, called FROMSAP, and a BCD file called HANS. The binary file consists of the standard 512-word heading, the displacements, the total stresses, the shear stresses, and the principal stresses all in three dimensions. If $\mathbf{n}$ is the number of node points and $m$ is the number of elements in the problem, the file can be described schematically as in the diagram at the sight.

The reader should note that the stresses are all given as being at the center of the element.

The BCD file is well labeled and should be selfexplanatory. When using the fast-solver, the variables used in the dynamic core allocation process are printed out. This should be of interest to the reader only if the code has memory expansion problems.
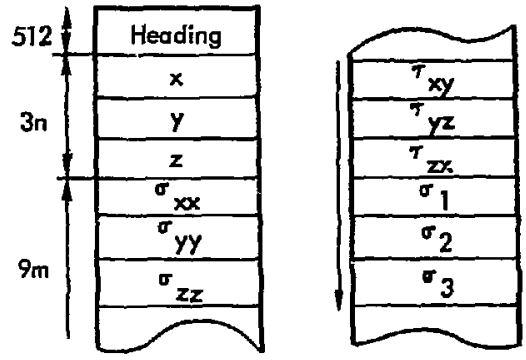


\section{Use of the Code}

In odder to run a problem. the ster will need the loaded SAP code and two input files. The binary input file LNK will notmally be the output of a niesh generator or of a vother cote and the BCD nife INDAT will be a card deck containing the boundary data. If only the name of the loaded file is typed in, the code will assume that it is to use all of LCM. If any message is included on the input line, SAP will limit its running size to less than 131,000 words.

The same procedure can be used when running tha code in production mode under ORDER, except that some etroi messages in SAP are sent to the controller. ORDER, however, interprets any message from a controllee as an ALL DONF, and will terminate the code before the high speed printer buffers are einptied. Hence the user may wish to use the simple controller that goes with the 3-D package, or write one of his own.

The SAP code makes heavy use of some LLL macro packsges. If the code is recompiled the user will need to place these macro files in his private tiles.

\section{PROBLEM SIZE LIMITATIONS}

Two factors determine how large a problen the code will handle. The more visible of these is the code's need to have in small core memory (SCM) coordinates, constraint information, material numbers, and temperature values for each node point. If $\boldsymbol{n}$ is the number of node points, then the amount of SCM for this storage is 1]n. In addition, the basic coding of the program requires approximately 15,000 words. SCM size is. of course, best determined at load time.

The more subtle size determination is made by the choice of solvers used in the code. The original solver of Wilson's code ${ }^{2}$ made no use of L.CM; with this solver optimal results are obtained when all of SCM is used, and only the usual 40,000-word LCM input/output $(1 / 0)$ buffer is needed. For the new solver, the optimal SCM workspace is equal to twice the half-bandwidth plus the size of the right-hand side of the equation. The determination of LCM sizes for this solver is completely dynamic except that the user can decide whether to run "large" or "small." The user should be aware that the LCM workspace limitation restrains the half-bandwidth of the problem to less than 582 .

SAP, in solving an implicit scheme with dynamic methods, creates many problems. One of these is the possible generation of excessively large files. To form the stiffness matrix the code must create a tile equal to $\mathrm{nm}$, where $\mathrm{n}$ is the number of node points and $\mathrm{m}$ is the half-bandwidth. On a heavily time-shared machine, the creation of such files is ill-advised, if not impossible. The user should be aware of these hardware constraints when designing his problem.

\section{PREPROCESSORS}

The following is a brief description of four small preprocessors that are frequently useful with the SAP program.

\section{CUBEGEN}

This program is a simple generator for SAP. The code is limited to providing a series of interfaced hexahedrons. The code must be given the number of blocks, the number of nodes total in the problem; and, for each block, the number of nodes in the block, the number of coordinates in the $x, y, z$ directions, and the starting and ending coordinates of each of these directions [FORMAT(215/4110/6F10.3)]. The code gives 
as output, LNK ard INDAT files. The bcindary conditions in INDAT are always simple node loads applied on an and surface. The code is useful in providing a series of different problems with varying bandwidths.

\section{DRGANIZER}

The code performs two functions: one is to reorganize the meshed turbine problem in order to lower the bandwidth, and the second is to generate the elements in the complicated, blocked data structure format. The code is used to provide input fo: the thermal diffusion code HEAT 3D. As as added benefit, is produces a restructured LNK file for SAP that lowers the bandwidth for the stress analysi : also. Input to the cade is merely a file with coordinates in the standard form.

\section{BOUNDER}

This program inserts boundary condition codes and material numbers into the bimary file, LNK. Input for the boundary conditions consists of a string of BCD cards with beginsing node, end node, how many nodes to skip, and flags determining whether $x . y$, or $z$ diretions ate fixed. After a blank card, the code assumes the same information will be given for the insertion of floating point materials numbers. The code will automatically expand disc files to accommodate the input information.

\section{FEVER}

This small code reads the time-dependent temperatures produced by the thermal diffusion program and inserts the temperatures into the LNX file for thermal stress analysis. Disc file sizes may be expanded as necessary.

\section{POSTPROCESSORS}

The following codes are useful in handling the output file, FROMSAP.

\section{L2NORMR}

This code scales the displacements and stresses found in SAP sc that the naximal stress satisfies the von Mise yield condition, and then forms the new coordinates. input to the code consists of the FROMSAP and LNK files for the problem. This code assumes that it will find the yield condition for the material as the 53rd word in the heading file. The program has been written entirely in the vector language and must be compiled and loaded appropriately. The user should be aware that this code will overwrite the FROMSAP file with its answer files; if the FROMSAP data are warted for later use, a copy of the file should be made.

\section{VPDX 3D}

Three dimensional computer graphics are provided by the VPIX 3D code. A number of options are available for analyzing and displaying results. 


\section{Appendix B}

HEMP 3D - A Finite Differenc: Code

for Calculating Elastic-Plastic Flow

\section{INTRODUCTION}

The HEMP 3D program can be used to solve problems in solid rrechanics involving dynemic plasticity and time dependent material behavior and problems in gas dynamics.

The equations of motion, the conservation equations. and the constitutive relations listed below are solved by finite difference methods following the format of the HEMP code. 1

\section{Equations of Motion}

$$
\begin{aligned}
& \rho \frac{\mathrm{d} \dot{\mathrm{x}}}{\mathrm{dt}}=\frac{\partial \Sigma_{\mathrm{xx}}}{\partial \mathrm{x}}+\frac{\partial \mathrm{T}_{\mathrm{xy}}}{\partial \mathrm{y}}+\frac{\partial \mathrm{T}_{\mathrm{zx}}}{\partial \mathrm{z}} \\
& \rho \frac{\mathrm{dy}}{\mathrm{dt}}=\frac{\partial \mathrm{T}_{\mathrm{xy}}}{\partial \mathrm{x}}+\frac{\partial \Sigma_{\mathrm{yy}}}{\partial \mathrm{y}}+\frac{\partial \mathrm{T}_{\mathrm{yz}}}{\partial \mathrm{z}} \\
& \rho \frac{\mathrm{d} \dot{\mathrm{z}}}{\mathrm{dt}}=\frac{\partial \mathrm{T}_{\mathrm{zx}}}{\partial \mathrm{x}}+\frac{\partial \mathrm{T}_{\mathrm{yz}}}{\partial \mathrm{y}}+\frac{\partial \Sigma_{\mathrm{zz}}}{\partial \mathrm{z}}
\end{aligned}
$$

Conservation of Mass and Energy

$$
\begin{aligned}
& \frac{d M}{d t}=0 \\
& M=\text { Mass element } \\
& \dot{E}=-(P+q) \dot{V}+V\left[s_{x x} \dot{\epsilon}_{x x}+s_{y y} \dot{\epsilon}_{y y}+s_{z z} \dot{\epsilon}_{z z}+T_{x y} \dot{\epsilon}_{x y}+T_{y z} \dot{\epsilon}_{y z}+T_{z x} \dot{\epsilon}_{z x}\right]
\end{aligned}
$$

\section{Constitutive Relations}

\section{Stresses}

\section{Stress deviators}

$$
\begin{aligned}
& \dot{s}_{\mathrm{xx}}=2 \mu\left(\dot{\epsilon}_{\mathrm{xx}}-\frac{1}{3} \frac{\dot{v}}{\mathrm{~V}}\right) \\
& \dot{\mathrm{s}}_{\mathrm{yy}}=2 \mu\left(\dot{\epsilon}_{\mathrm{yy}}-\frac{1}{3} \frac{\dot{\mathrm{v}}}{\mathrm{v}}\right) \\
& \dot{\mathrm{s}}_{\mathrm{zz}}=2 \mu\left(\dot{\epsilon}_{\mathrm{zz}}-\frac{1}{3} \frac{\dot{\mathrm{v}}}{\mathrm{v}}\right)
\end{aligned}
$$




\section{Shear stresses}

$$
\begin{aligned}
& \dot{\mathrm{T}}_{\mathrm{xy}}=\mu\left(\dot{\mathrm{E}}_{\mathrm{xy}}\right) \\
& \dot{\mathrm{T}}_{\mathrm{zx}}=\mu\left(\dot{\varepsilon}_{\mathrm{zx}}\right) \\
& \dot{\mathrm{T}}_{\mathrm{yz}}=\mu\left(\dot{\epsilon}_{\mathrm{yz}}\right) \\
& \mu=\text { shear modulus }
\end{aligned}
$$

\section{Total stresses}

$$
\begin{aligned}
& \Sigma_{x x}=-(P+q)+s_{x x} \\
& \Sigma_{y y}=-(P+q)+s_{y y} \\
& \Sigma_{z z}=-(P+q)+s_{z z}
\end{aligned}
$$

\section{Artificial viscosity}

$$
\begin{aligned}
& q=\frac{\rho^{0}}{v}\left[C_{A}^{2} \ell^{2}\left(\frac{\dot{V}}{v}\right)^{2}+C_{L} \ell a \frac{\dot{v}}{v}\right] \\
& C_{A} \text { and } C_{L} \text { are constants } \\
& \ell=\text { characteristic grid length } \\
& a=\text { characteristic velocity. }
\end{aligned}
$$

\section{Velocity Strains}

$$
\begin{aligned}
& \dot{\epsilon}_{x x}=\frac{\partial \dot{x}}{\partial x}, \dot{\epsilon}_{y y}=\frac{\partial \dot{y}}{\partial y}, \dot{\epsilon}_{z z}=\frac{\partial \dot{z}}{\partial z} \\
& \dot{\epsilon}_{x y}=\left(\frac{\partial \dot{x}}{\partial y}+\frac{\partial \dot{y}}{\partial x}\right): \dot{\epsilon}_{z x}=\left(\frac{\partial \dot{x}}{\partial z}+\frac{\partial \dot{z}}{\partial x}\right): \dot{\epsilon}_{y z}=\left(\frac{\partial \dot{y}}{\partial z}+\frac{\partial \dot{z}}{\partial y}\right)
\end{aligned}
$$

\section{Hydrostatic Pressure}

$$
\begin{aligned}
P & =a(\eta-1)+b(\eta-1)^{2}+c(\eta-1)^{3}+d \eta E \\
\eta & =\frac{1}{V}=\rho / \rho^{0}, \text { where } a, b, c \text { and } d \text { are equation of state constants } \\
\rho & =\text { actual density } \\
\rho^{0} & =\text { reference density } \\
E & =\text { internal energy per original volume. }
\end{aligned}
$$


von Mises Yield Condition

$$
\begin{aligned}
& \sqrt{2 J}-\sqrt{\frac{2}{3}} Y \leqslant 0 \\
& Y=a\left(b+\bar{\epsilon}^{\mathrm{p}}\right)^{\mathrm{c}} \\
& \bar{\epsilon}^{\mathrm{P}}=\text { equivalent plastic strain } \\
& 2 J=\text { Second invariant of the deviatoric stress tensor } \\
& \mathrm{a}, \mathrm{b} \text { and c are flow stress constants. }
\end{aligned}
$$

\section{FINITE DIFFERENCE EQUATIONS FOR HEMP 30}

The three dimensional difference operators used to approximate the partial derivatives of the equations given above are given schematically in Ref. 3 . The complete details are given here.

The physical object is divided into zones defined by cight grid points, Fig. B-1. The grid, (i, $j, k)$ moves with the material and the mass within a zone remains constant. In the notations that follow a superscript refers to the time centering of a parameter or equation and the subscript refers to the space centering.

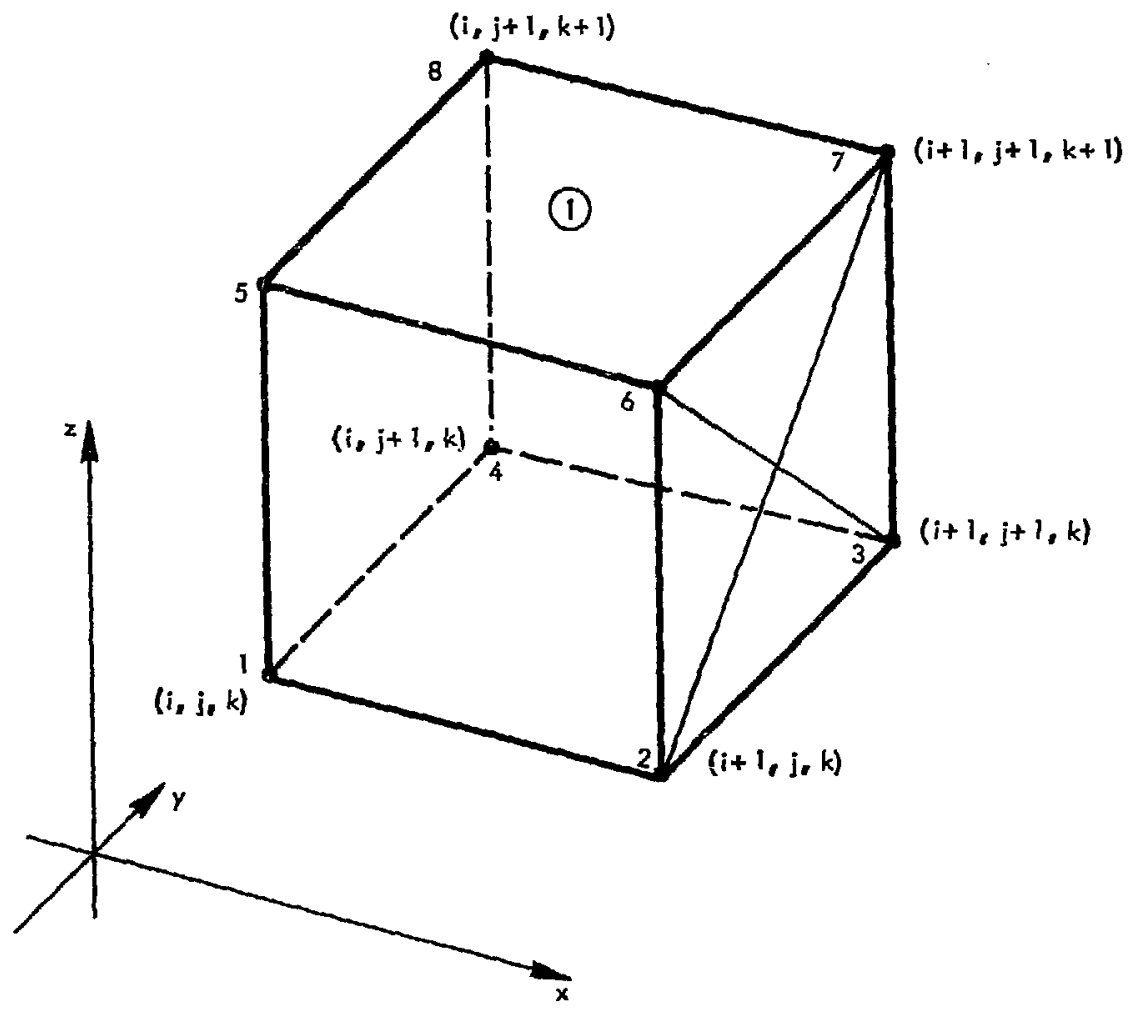

Fig. B-I. Grid and numbering scheme for Zone (1). 


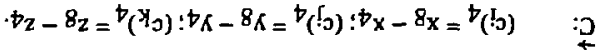

$$
\begin{aligned}
& \cdot D_{Z}-I_{Z}={ }^{D}\left({ }^{I} q\right) ! t_{K}-I_{K}={ }^{D}\left(I_{q}\right) ! t_{X}-I_{X}={ }^{D}\left(I_{q}\right) \quad: g
\end{aligned}
$$

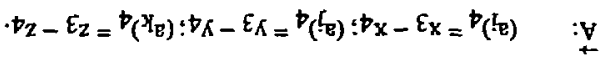

$$
\begin{aligned}
& \text { spuวuoduoว }
\end{aligned}
$$

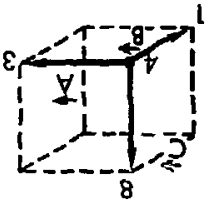

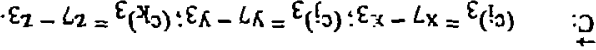

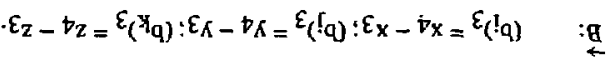

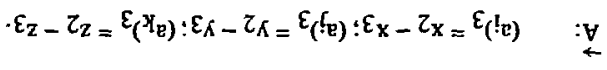

$$
\begin{aligned}
& \text { s1นวนั0duoว }
\end{aligned}
$$

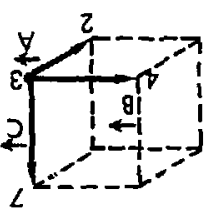

$\overline{\varepsilon=8}$

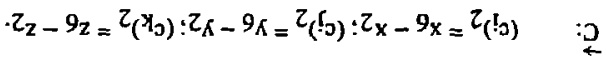

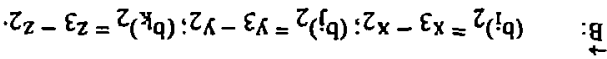

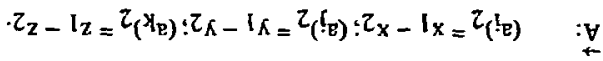

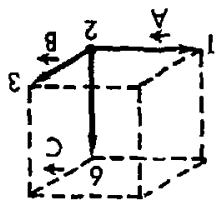

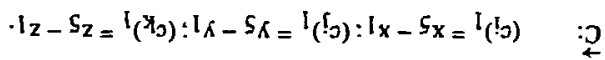

$$
\begin{aligned}
& \left.\cdot I_{z}-\tau_{z}=I_{(} I_{q}\right) \div I_{K}-\tau_{K}=I_{(}\left(I_{q}\right): I_{x}-z_{x}=I_{(}\left(I_{q}\right) \quad: g
\end{aligned}
$$

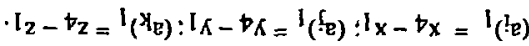

$$
\begin{aligned}
& \text { stuauodwoJ }
\end{aligned}
$$

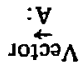

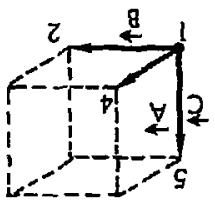

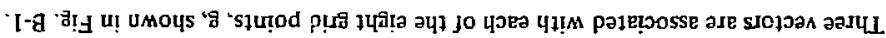




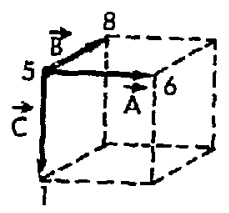
Vector
Components
$\overrightarrow{\mathrm{A}}$ :
$\left(a_{i}\right)_{5}=x_{6}-x_{5}:\left(a_{j}\right)_{5}=y_{0}-y_{5}:\left(a_{k}\right)_{5}=z_{6}-z_{5}$.
$\overrightarrow{\mathrm{B}}$
$\left(b_{i}\right)_{5}=x_{8}-x_{5}:\left(b_{j}\right)_{5}=y_{8}-y_{5}:\left(b_{k}\right)_{5}=z_{8}-z_{5}$.
$\vec{c}:$
$\left(c_{j}\right)_{5}=x_{1}-x_{5}:\left(c_{j}\right)_{5}=y_{1}-y_{5}:\left(c_{k}\right)_{5}=z_{1}-z_{5}$.

$\underline{g=6}$

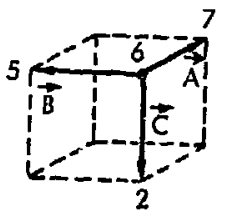
Vector
Components
$\vec{A}$ :
$\left(a_{i}\right)_{6}=x_{7}-x_{6}:\left(a_{j}\right)_{6}=y_{7}-y_{6}:\left(a_{k}\right)_{6}=z_{7}-z_{6}$.
$\overrightarrow{\mathbf{B}}$ :
$\left(b_{i}\right)_{6}=x_{5}-x_{6}:\left(b_{j}\right)_{6}=y_{5}-y_{6} ;\left(b_{k}\right)_{6}=z_{5}-z_{6}$
$\overrightarrow{\mathrm{C}}$
$\left(c_{i}\right)_{6}=x_{2}-x_{6} ;\left(c_{j}\right)_{6}=y_{2}-y_{6} ;\left(c_{k}\right)_{6}=z_{2}-z_{6}$.

$g=7$

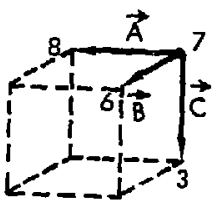
Vector
Components
$\vec{A}$ :
$\left(a_{i}\right)_{7}=x_{8}-x_{7} ;\left(a_{j}\right)_{7}=y_{8}-y_{7} ;\left(a_{k}\right)_{7}=z_{8}-z_{7}$.
$\vec{B}$
$\left(b_{i}\right)_{7}=x_{6}-x_{7} ;\left(b_{j}\right)_{7}=y_{6}-y_{7} ;\left(b_{k}\right)_{7}=z_{6}-z_{7}$.
$\overrightarrow{\mathbf{C}}$ :
$\left(c_{i}\right)_{7}=x_{3}-x_{7}:\left(c_{j}\right)_{7}=y_{3}-y_{7}:\left(c_{k}\right)_{7}=z_{3}-z_{7}$.

$\underline{\mathrm{g}=8}$

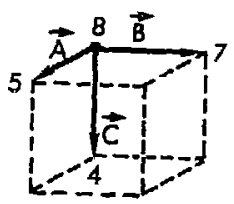
Vector
Components
$\vec{A}$ :
$\left(a_{j}\right)_{8}=x_{5}-x_{8} ;\left(a_{j}\right)_{8}=y_{5}-y_{8}:\left(a_{k}\right)_{8}=z_{5}-z_{8}$.
$\vec{B}: \quad\left(b_{i}\right)_{8}=x_{7}-x_{8} ;\left(b_{j}\right)_{8}=y_{7}-y_{8} ;\left(b_{k}\right)_{8}=z_{7}-z_{8}$
$\overrightarrow{\mathrm{c}}: \quad\left(c_{\mathrm{i}}\right)_{8}=\mathrm{x}_{4}-\mathrm{x}_{8} ;\left(c_{j}\right)_{8}=y_{4}-y_{8} ;\left(c_{k}\right)_{8}=z_{4}-z_{8}$. 
Refer to Fig. B-1.

$$
\text { "(1) }=\frac{1}{8} \sum_{\mathrm{g}=1}^{8}[\overrightarrow{\mathrm{B}} \times \overrightarrow{\mathrm{A}} \cdot \overrightarrow{\mathrm{C}}]
$$

where:

$$
\vec{B} \times \vec{A} \cdot C]_{g=1}^{n}=\left\{\begin{array}{l}
b_{i} b_{j} b_{k} \\
a_{i} a_{j} a_{k} \\
c_{i} c_{j} c_{k}
\end{array}\right\}_{g=1}=\left[b_{i}\left(a_{j} c_{k}-a_{k} c_{j}\right)-b_{j}\left(a_{i} c_{k}-a_{k} c_{j}\right)+b_{k}\left(a_{i} c_{j}-a_{j} c_{j}\right)\right]_{g=1}^{n}
$$

Kepeat for $g=2 \rightarrow 8$

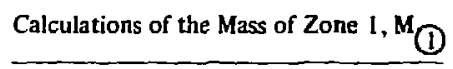

$$
\begin{aligned}
& M_{(1)}=\left[\frac{\rho^{0}}{v^{0}} v^{0}\right]_{(D} \\
& \rho^{0}=\text { reference density } \\
& v^{0}=\text { initial relative volume } \\
& v^{0}=\text { actual volume calculated from the coordinates at time } t=0 .
\end{aligned}
$$

\section{Conservations of Mass}

$$
\begin{aligned}
& v_{(1)}^{n}=\left(\frac{\rho^{0}}{M}\right)_{(1)} \text { (1) } \text {; where } v_{(1)}^{n} \text { is the volume at time } t=n \text { and } v_{(1)}^{n} \text { is the relative volume. Similarly, } \\
& v_{(1)}^{n+1}=\left(\frac{\rho^{0}}{M}\right)_{(1)}{ }_{(1)}^{n+1} \text { where the volume } v^{n+1} \text { is calculated from the coordinates at time } n+1 \text {. } \\
& v_{(1)}^{n+1 / 2}=\frac{1}{2}\left(v^{n+1}+v^{n}\right)-\text { definition of relative volume at } t=n+1 / 2 \text {. }
\end{aligned}
$$

\section{Equations of Motion}

The following acceleration equations are applied to point 0 in Fig. B-2. 


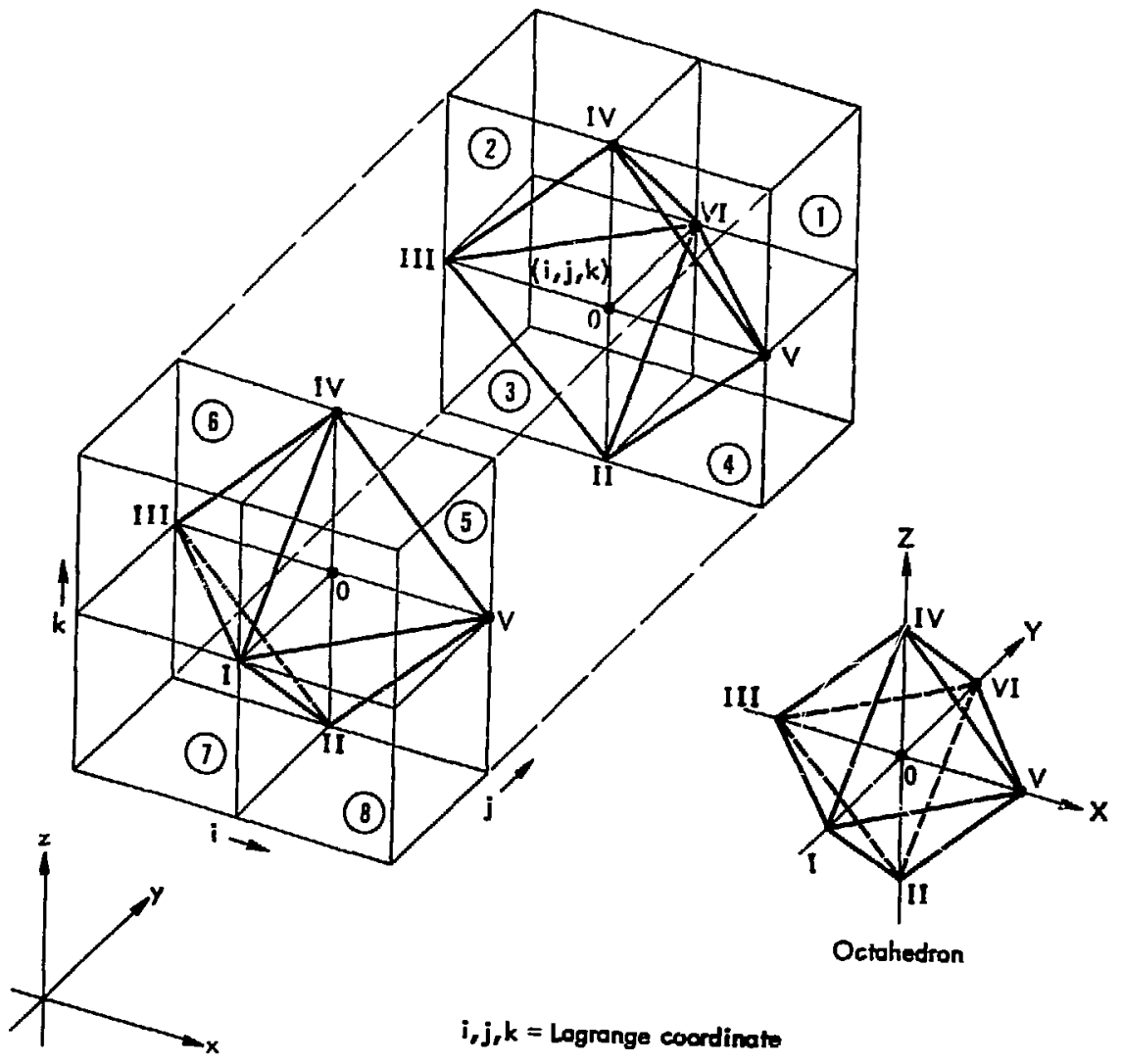

Fig. B-2. Grid for accelerating point $(i, j, k)$.

Mass Associated with Point $(i, j, k)$

$$
\left.(\Phi)_{i, j, x}=\frac{1}{8} i M_{(1)}+M_{(2)}+M_{(3)}+M_{(4)}+M_{(5)}+M_{(6)}+M_{(7)}+M_{(8)}\right]
$$

Motion in the $\mathrm{x}$ Direction

$$
\left(\frac{d \dot{x}}{d t}\right)_{i, j, k}=\frac{1}{\rho_{i, j, k}^{n}}\left[\frac{\partial \Sigma_{x x}}{\partial x}+\frac{\partial T_{x y}}{\partial y}+\frac{\partial T_{z x}}{\partial z}\right]_{i, j, k}^{n} \text {, where }
$$




$$
\begin{aligned}
& \left(\frac{1}{\rho} \frac{\partial \Sigma_{x x}}{\partial x}\right)_{i, j, k}^{n}=\frac{1}{4 \Phi_{i, j, k}}\left\{\left(\Sigma_{x x}\right)_{(1)}\left[\left(y_{V I}-y_{V}\right)\left(z_{I V}-z_{V}\right)-\left(z_{V I}-z_{V}\right)\left(y_{I V}-y_{V}\right)\right]\right. \\
& +\left(\Sigma_{x x}\right)_{(4)}\left[\left(y_{11}-y_{V}\right)\left(z_{V I}-z_{V}\right)-\left(z_{1 I}-z_{V}\right)\left(y_{V I}-y_{V}\right)\right\} \\
& +\left(\Sigma_{x x}\right)_{(2)}^{\left[\left(y_{I V}-y_{I I I}\right)\left(z_{V I}-z_{I I I}\right)-\left(z_{I V}-z_{I I I}\right)\left(y_{V I}-y_{I I I}\right)\right]} \\
& +\left(\Sigma_{x x}\right)_{(3)}\left[\left(y_{V i}-y_{I I I}\right)\left(z_{I I}-z_{I I I}\right)-\left(z_{V I}-z_{I I I}\right)\left(y_{I I}-y_{I I I}\right)\right] \\
& +\left(\Sigma_{x x}\right)_{(5)}\left[\left(y_{V}-y_{1}\right)\left(z_{I V}-z_{I}\right)-\left(2 V-z_{1}\right)\left(y_{I V}-y_{I}\right)\right] \\
& +\left(\Sigma_{x x}\right]_{(8)}\left[\left(y_{11}-y_{1}\right)\left(z_{V}-z_{1}\right)-\left(z_{1 I}-z_{1}\right)\left(y_{V}-y_{1}\right)\right] \\
& +\left(\Sigma_{x x}\right]_{(6)}\left[\left(y_{I}-y_{I I I}\right)\left(z_{I V}-z_{I I I}\right)-\left(z_{I}-z_{I I I}\right)\left(y_{I V}-y_{I I I}\right)\right] \\
& \left.+\left(\Sigma_{x x}\right)_{(7)}\left[\left(y_{I I}-y_{I I I}\right)\left(z_{I}-z_{I I I}\right)-\left(z_{I I}-z_{I I I}\right)\left(y_{I}-y_{I I I}\right)\right]\right\}_{i, j, k}^{n}
\end{aligned}
$$

To form $\left(1 / p \partial T_{x y} / \partial y\right)_{i, j, k}^{n}$, ieplace each $\Sigma_{x x}$ in the right side of the athne expression with $T_{x y}$, every $y$ with the corresponding $z$, and each $z$ with the corresponding $x$.

To form $\left(1 / p \partial T_{z x} / \partial z\right)_{i, j, k}^{n}$, replace each $\Sigma_{x x}$ in the above expression with $T_{z x}$, every $y$ with the corresponding $x$ and every $z$ with the corresponding $y$.

The $x$-direction velocity at $n+1 / 2$ and positions at times $n+1$ and $n+1 / 2$ are:

$$
\begin{aligned}
& \dot{x}_{i, j, k}^{n+1 / 2}=\dot{x}_{i, j, k}^{n-1 / 2}+\left(\frac{d \dot{x}}{d t}\right)_{i, j, k}^{n} \Delta t^{n} \\
& x_{i, j, k}^{n+1}=x_{i, j, k}^{n}+\dot{x}_{i, j, k} \Delta t^{n+1 / 2} \\
& x_{i, j, k}^{n+1 / 2}=\frac{1}{2}\left(x_{i, j, k}^{n+1}+x_{i, j, k}^{n}\right) .
\end{aligned}
$$

\section{Motion in the y Direction}

$$
\begin{aligned}
& \left(\frac{d y}{d t}\right)_{i, j, k}^{n}=\frac{1}{\rho_{i, j, k}^{n}}\left[\frac{\partial T_{x y}}{\partial x}+\frac{\partial \Sigma_{y y}}{\partial y}+\frac{\partial T_{y z}}{\partial z}\right]_{i, j, k}^{n} \text {; where } \\
& \left(\frac{1}{\rho} \frac{\partial T_{x y}}{\partial x}\right)_{i, j, k}^{n}=\begin{array}{c}
\text { parme as }\left(1 / \rho \partial \Sigma_{x x} / \partial x\right)_{i, j, k}^{n} \text { ponding value of } T_{x y} \\
\left(\frac{1}{\rho} \frac{\partial \Sigma_{y y}}{\partial y}\right)_{i, j, k}^{n}=\begin{array}{c}
\text { same as }\left(I / \rho \partial T_{x y} / \partial y\right)_{i, j, k}^{n} \\
\text { ponding value of } \Sigma_{y y}
\end{array}
\end{array}
\end{aligned}
$$




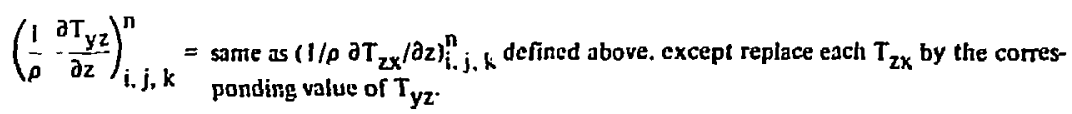

The $y$-dircction velocity at time $n+1 / 2$ and positions at times $n+1$ and $n+1 / 2$ are:

$$
\begin{aligned}
& \dot{y}_{i, j, k}^{n+1 / 2}=\dot{y}_{i, j, k}^{n-1 / 2}+\left(\frac{d \dot{y}}{d t}\right)_{i, j, k}^{n} \Delta t^{n} \\
& y_{i, j, k}^{n+1}=y_{i, j, k}^{n}+\dot{y}_{i, j, k}^{n+1 / 2} \Delta t^{n+1 / 2} \\
& y_{i, j, k}^{n+1 / 2}=\frac{1}{2}\left(y_{i, j, k}^{n+1}+y_{i, j, k}^{n}\right) .
\end{aligned}
$$

\section{Motion in the z Direction}

$$
\begin{aligned}
& \left(\frac{d \dot{z}}{d t}\right)_{i, j . k}^{n}=\frac{1}{\rho_{i, j, k}^{n}}\left[\frac{\partial T_{z x}}{\partial x}+\frac{\partial T_{y z}}{\partial y}+\frac{\partial \Sigma_{z z}}{\partial z}\right]_{i, j, k}^{n}
\end{aligned}
$$

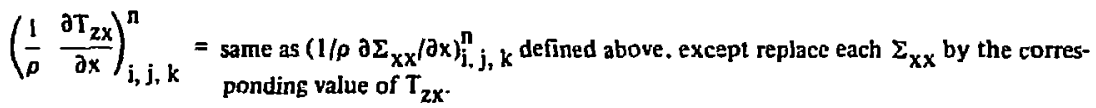

$$
\begin{aligned}
& \left(\frac{1}{\rho} \frac{\partial T_{y z}}{\partial y}\right)_{i, j, k}^{n}=\begin{array}{l}
\text { same as }\left(1 / \rho \partial T_{x y} / \partial y\right)_{i, j, k}^{n} \\
\text { ponding value of } T_{y z}
\end{array}
\end{aligned}
$$

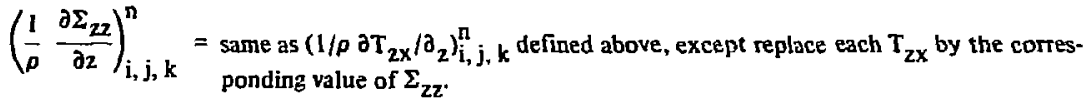

The $z$-direction velocity at time $n+1 / 2$ and positions at times $n+1$ and $n+1 / 2$ are:

$$
\begin{aligned}
& i_{i, j, k}^{n+1 / 2}=i_{i, j, k}^{n-1 / 2}+\left(\frac{d \dot{z}}{d t}\right)_{i, j, k}^{n} \Delta t^{n} \\
& z_{i, j, k}^{n+1}=z_{i, j, k}^{n}+z_{i, j, k}^{n+1 / 2} \Delta t^{n+l / 2} \\
& z_{i, j, k}^{n+l / 2}=\frac{1}{2}\left(z_{i, j, k}^{n+1}+z_{i, j, k}^{n}\right)
\end{aligned}
$$




\section{Calculation of Incremental Strains}

The finite difference mapping procedure to calculate the surfacc ' surface in units of triangles. The velocity assaciated with a given triangle is taken as the average of the velocities defined at the triangle comers. The triangular surface area vectors are calculated to poirt out of the zone surface The dot product of the area vector with the direction vector multiplied by the average velocity gives the velocity flux through the surface in the given direction. The mapping procedure actually covers the zor:e surface area.

Fig. B-1, two times. The difference equations used to calculate

$$
\frac{\partial \dot{x}}{\partial x} \frac{\partial \dot{x}}{\partial y} \text { and } \frac{\partial \dot{x}}{\partial z} \text { are given explicitly below. }
$$

The remaining velocity derivatives required to calculate the components of strain are calculated by replacing $\dot{x}$ in those equations by $\dot{y}$ and then by $i$ so as to complete the set:

$$
\left|\begin{array}{lll}
\frac{\partial \dot{x}}{\partial x} & \frac{\partial \dot{x}}{\partial y} & \frac{\partial \dot{x}}{\partial z} \\
\frac{\partial \dot{y}}{\partial x} & \frac{\partial \dot{y}}{\partial y} & \frac{\partial \dot{y}}{\partial z} \\
\frac{\partial \dot{z}}{\partial x} & \frac{\partial \dot{z}}{\partial y} & \frac{\partial \dot{z}}{\partial z}
\end{array}\right|
$$

Velocity Derivatives Corresponding to Zone (D, Fig. B-1.

$$
\left(\frac{\partial \dot{x}}{\partial x}\right)_{(1)}^{n+1 / 2}=\left(\frac{1}{12 \nu^{n+1 / 2}}\right) \sum_{\mathrm{g}=1}^{8}\left[\dot{x}_{A B}(\vec{A} \times \vec{B}) \cdot \vec{i}+\dot{x}_{C A}(\vec{C} \times \vec{A}) \cdot \vec{i}+\dot{x}_{B C}(\vec{B} \times \vec{C}) \cdot \vec{i}\right]_{g}^{n+1 / 2}
$$

where

$$
\begin{aligned}
& \left(\dot{x}_{A B}\right)_{g=1}=\left(\dot{x}_{1}+\dot{x}_{2}+\dot{x}_{4}\right),\left(\dot{x}_{C A}\right)_{g=1}=\left(\dot{x}_{1}+\dot{x}_{4}+\dot{x}_{5}\right),\left(\dot{x}_{B C}\right)_{g=1}=\left(\dot{x}_{1}+\dot{x}_{2}+\dot{x}_{5}\right) . \\
& (\vec{A} \times \vec{B} \cdot \vec{i})_{g=1}=\left|\begin{array}{lll}
1 & 0 & 0 \\
a_{i} & a_{j} & a_{k} \\
b_{i} & b_{j} & b_{k}
\end{array}\right|_{g=1}=\left[a_{i}\left(a_{j} b_{k}-a_{k} b_{j}\right)\right]_{g=1} . \\
& (\vec{C} \times \vec{A} \cdot \vec{i})_{g=1}=\left|\begin{array}{lll}
1 & 0 & 0 \\
c_{i} & c_{j} & c_{k} \\
a_{i} & a_{j} & a_{k}
\end{array}\right|_{E=1}=\left[c_{i}\left(c_{j} a_{k}-c_{k} a_{j}\right)\right]_{g=1} .
\end{aligned}
$$




$$
(\vec{B} \times \vec{C} \cdot \vec{i})_{g=1}=\left|\begin{array}{ccc}
1 & 0 & 0 \\
b_{i} & b_{j} & b_{k} \\
c_{i} & c_{j} & c_{k}
\end{array}\right|_{g=1}=\left[b_{j}\left(b_{j} c_{k}-b_{k} c_{j}\right)\right]_{g=1}
$$

The above steps, written for $g=1$, must be repeated for $g=2 \rightarrow 8$.

$$
\left(\frac{\partial \dot{x}}{\partial y}\right)_{(1)}^{n+1 / 2}=\left(\frac{1}{12 y_{(D)}^{n+1 / 2}}\right) \sum_{\mathrm{g}=1}^{\delta}\left[x_{s}(\vec{A} \times \vec{B}) \cdot \vec{j}+\dot{x}_{C A}(\vec{C} \times \vec{A}) \cdot \vec{j}+\dot{x}_{B C}(\vec{B} \times \vec{C}) \cdot \vec{j}\right]_{g}^{n+1 / 2}
$$

where

$$
\begin{aligned}
& \left(\dot{x}_{A B}\right)_{g=1},\left(\dot{x}_{C A}\right)_{g=1} \text {, and }\left(\dot{x}_{B C}\right)_{g=1} \text { are as defined above. } \\
& \left(\vec{A} \times \vec{B} \cdot \vec{j}_{g=1}=\left|\begin{array}{lll}
0 & 1 & 0 \\
a_{i} & a_{j} & a_{k} \\
b_{i} & b_{j} & b_{k}
\end{array}\right|_{g=1}=\left[-a_{j}\left(a_{i} b_{k}-a_{k} b_{j}\right)\right]_{g=1}\right. \\
& (\vec{C} \times \vec{A} \cdot \vec{j})_{g=1}=\left|\begin{array}{lll}
0 & 1 & 0 \\
c_{i} & c_{j} & c_{k} \\
a_{i} & a_{j} & a_{k}
\end{array}\right|_{g=1}=\left[-c_{j}\left(c_{i} a_{k}-c_{k} a_{i j}\right)\right]_{g=1} . \\
& (\vec{B} \times \vec{C} \cdot \vec{j})_{g=1}=\left|\begin{array}{lll}
0 & 1 & 0 \\
b_{i} & b_{j} & b_{k} \\
c_{j} & c_{k} & c_{j}
\end{array}\right|_{g=1}=\left[-b_{j}\left(b_{i} c_{j}-b_{k} c_{j}\right)\right]_{g=1} .
\end{aligned}
$$

The above steps, written for $\mathrm{g}=1$, must be repeated for $\mathrm{g}=2 \rightarrow 8$.

$$
\left(\frac{\partial \dot{x}}{\partial z}\right)_{(1)}^{n+1 / 2}=\left(\frac{1}{12 v \frac{n+1 / 2}{(1)}}\right) \sum_{g=1}^{8}\left[\dot{x}_{A B}(\vec{A} \times \vec{B}) \cdot \vec{k}+\dot{x}_{C A}(\vec{C} \times \vec{A}) \cdot \vec{k}+\dot{x}_{B C}(\vec{B} \times \vec{C}) \cdot \vec{k}\right]_{g}^{n+1 / 2}
$$

where

$$
\begin{aligned}
& \left(\dot{x}_{A B}\right)_{g=1},\left(\dot{x}_{C A}\right)_{g=1} \text {, and }\left(\dot{x}_{B C}\right)_{g=1} \text { are as defined above. } \\
& (\vec{A} \times \vec{B} \cdot \vec{k})_{g=1}=\left|\begin{array}{ccc}
0 & 0 & 1 \\
a_{i} & a_{j} & a_{k} \\
b_{i} & b_{j} & b_{k}
\end{array}\right|_{g=1}=\left[a_{k}\left(a_{i} b_{j}-a_{j} b_{j}\right)\right]_{g=1}
\end{aligned}
$$




$$
\begin{aligned}
& \overrightarrow{(\vec{C}} \times \vec{A} \cdot \vec{k})_{g=1}=\left|\begin{array}{ccc}
0 & 0 & 1 \\
c_{j} & c_{j} & c_{k} \\
a_{i} & a_{j} & a_{k}
\end{array}\right|_{g=1}=\left[c_{k}\left(c_{i} a_{j}-c_{j} a_{i}\right]_{g=l}\right. \\
& \overrightarrow{\boldsymbol{B}} \times \overrightarrow{\mathrm{C}} \cdot \vec{k})_{g=1}=\left|\begin{array}{ccc}
0 & 0 & 1 \\
b_{i} & b_{j} & b_{k} \\
c_{j} & c_{j} & c_{k}
\end{array}\right|_{g=1}=\left\{b_{k}\left(b_{i} c_{j}-b_{j} c_{i}\right\}\right\}_{g=1}
\end{aligned}
$$

The above steps, written for $g=1$, must be repeated for $g=2 \rightarrow 8$.

$\frac{\partial \dot{y}}{\partial x}=$ same as $\partial \dot{x} / \partial x$ except replace $\dot{x}$ by the corresponding $\dot{y}$

$\frac{\partial \dot{z}}{\partial x}=$ same as $\partial \dot{x} / \partial x$ except replace $\dot{x}$ by the corsesponding $\dot{z}$

$\frac{\partial \dot{y}}{\partial y}=$ same as $\partial \dot{x} / \partial y$ except replace $\dot{x}$ by the corresponding $\dot{y}$

$\frac{\partial \dot{z}}{\partial y}=$ same as $\partial \dot{x} / \partial y$ except replace $\dot{x}$ by the corresponding $\dot{z}$

$\frac{\partial \dot{y}}{\partial z}=$ same as $\partial \dot{x} / \partial z$ except replace $\dot{x}$ by the corresponding $\dot{y}$

$\frac{\partial \dot{z}}{\partial z}=$ same as $\partial \dot{x} / \partial z$ except teplace $\dot{x}$ by the corresponding $\dot{z}$

\section{Incremental Strains}

$$
\begin{aligned}
& \left(\Delta \varepsilon_{x x}\right)_{(1)}^{n+1 / 2}=\left(\frac{\partial \dot{x}}{\partial x}\right)_{(1)}^{n+1 / 2} \Delta t^{n+1 / 2} \\
& \left(\Delta \varepsilon_{y y}\right)_{(1)}^{n+1 / 2}=\left(\frac{\partial \dot{y}}{\partial y}\right)_{(1)}^{n+1 / 2} \Delta t^{n+1 / 2} \\
& \left(\Delta \varepsilon_{z z}\right)_{(1)}^{n+1 / 2}=\left(\frac{\partial \dot{z}}{\partial z}\right)_{(1)}^{n+1 / 2} \Delta t^{n+1 / 2} \\
& \left(\Delta \varepsilon_{x y}\right)_{(1)}^{n+1 / 2}=\left[\left(\frac{\partial \dot{x}}{\partial y}\right)_{(1)}^{n+1 / 2}+\left(\frac{\partial \dot{y}}{\partial x}\right)_{(1)}^{n+1 / 2}\right] \Delta t^{n+1 / 2}
\end{aligned}
$$




$$
\begin{aligned}
& \left(\Delta \varepsilon_{y z}\right)_{(1)}^{n+1 / 2}=\left[\left(\frac{\partial \dot{y}}{\partial z}\right)_{(1)}^{n+1 / 2}+\left(\frac{\partial \dot{z}}{\partial y}\right)_{(1)}^{n+1 / 2}\right] \Delta t^{n+1 / 2} \\
& \left(\Delta \varepsilon_{z x}\right)_{(1)}^{n+1 / 2}=\left[\left(\frac{\partial \dot{z}}{\partial x}\right)_{(1)}^{n+1 / 2}+\left(\frac{\partial \dot{x}}{\partial z}\right)_{(1)}^{n+1 / 2}\right] \Delta t^{n+1 / 2} \\
& \left(\frac{\Delta v}{v}\right)_{(1)}^{n+1 / 2}=2\left(\frac{v_{(1)}^{n+1}-v_{(1)}^{n}}{v_{(1)}^{n+1}+v_{(1)}^{n}}\right)
\end{aligned}
$$

\section{Calculation of Stress}

\section{Stress Deviators}

$$
\begin{aligned}
& \left(s_{x x}\right)_{(1)}^{n+1}=\left(s_{x x}\right)_{(1)}^{n}+2 \mu\left[\left(\Delta \varepsilon_{x x}\right)_{(1)}^{n+1 / 2}-\frac{1}{3}\left(\frac{\Delta V}{V}\right)_{(1)}^{n+1 / 2}\right]+\left(\delta_{x x}^{n}\right)_{(1)} \\
& \left(s_{y y}\right)_{(1)}^{n+1}=\left(s_{y y}\right)_{(1)}^{n}+2 \mu\left[\left(\Delta \varepsilon_{y y}\right)_{(1)}^{n+1 / 2}-\frac{1}{3}\left(\frac{\Delta V}{V}\right)_{(1)}^{n+1 / 2}\right]+\left(\delta_{y y}^{n}\right)_{(1)} \\
& \left(s_{z z}\right)_{(1)}^{n+1}=\left(s_{z z}\right)_{(1)}^{n}+2 \mu\left[\left(\Delta \varepsilon_{z z}\right)_{(1)}^{n+1 / 2}-\frac{1}{3}\left(\frac{\Delta V}{V}\right)_{(1)}^{n+1 / 2}\right]+\left(\delta_{z z}^{n}\right)_{(1)} \\
& \left(T_{x y}\right)_{(1)}^{n+1}=\left(T_{x y}\right)_{(1)}^{n}+\mu\left(\Delta \varepsilon_{x y}\right)_{(1)}^{n+1 / 2}+\left(\delta_{x y}^{n}\right)_{(1)} \\
& \left(T_{y z}\right)_{(1)}^{n+1}=\left(T_{y z}\right)_{(1)}^{n}+\mu\left(\Delta \varepsilon_{y z}\right)_{(1)}^{n+1 / 2}+\left(\delta_{y z}^{n}\right)_{(1)} \\
& \left(T_{z x}\right)_{(1)}^{n+1}=\left(T_{z x}\right)_{(1)}^{n}+\mu\left(\Delta \varepsilon_{z x}\right)_{(1)}^{n+1 / 2}+\left(\delta_{z x}^{n}\right)
\end{aligned}
$$

Note: The terms $\delta$ tisat have been added to the stress deviators are corrections for zone rotations. If a zone has rotated during the time interval $\Delta \mathrm{t}^{\mathrm{n}+1 / 2}=\mathrm{t}^{\mathrm{n}+1}-\mathrm{t}^{\mathrm{n}}$ the stresses at time $\mathrm{t}^{\mathrm{n}}$ must be recalculated so that they will be referred to the $x, y, z$ coordinate systems in their new positions. ${ }^{1}$ The correction terms are given by

$$
\begin{aligned}
& \delta_{x x}^{n}=-2 \omega_{z}^{n} T_{x y}^{n}+2 \omega_{y}^{n} T_{z x}^{n}, \\
& \delta_{y y}^{n}=+2 \omega_{z}^{n} T_{x y}^{n}-2 \omega_{x}^{n} T_{y z}^{n},
\end{aligned}
$$




$$
\begin{aligned}
& \delta_{z z}^{n}=+2 \omega_{x}^{n} T_{y z}^{n}-2 \omega_{y}^{n} T_{z x}^{n}=-\delta_{y y}^{n}-\delta_{x x}^{n}, \\
& \delta_{x y}^{n}=\omega_{z}^{n}\left(s_{x x}^{n}-s_{y y}^{n}\right)+\omega_{y}^{n} T_{y z}^{n}-\omega_{x}^{n} T_{z x}^{n}, \\
& \delta_{y z}^{n}=\omega_{x}^{n}\left(s_{y y}^{n}-s_{z z}^{n}\right)+\omega_{z}^{n} T_{z x}^{n}-\omega_{y}^{n} T_{x y}^{n}, \\
& \delta_{z x}^{n}=\omega_{y}^{n}\left(s_{z z}^{n}-s_{x x}^{n}\right)+\omega_{x}^{n} T_{x y}^{n}-\omega_{z}^{n} T_{y z}^{n},
\end{aligned}
$$

where:

$$
\begin{aligned}
& \omega_{x}^{\bar{n}}=\frac{1}{2}\left(\frac{\partial \dot{z}}{\partial y}-\frac{\partial \dot{y}}{\partial z}\right) \Delta t^{n+1 / 2}, \\
& \omega_{y}^{n}=\frac{1}{2}\left(\frac{\partial \dot{x}}{\partial z}-\frac{\partial \dot{z}}{\partial x}\right) \Delta t^{n+1 / 2}, \\
& \omega_{z}^{n}=\frac{1}{2}\left(\frac{\partial \dot{y}}{\partial x}-\frac{\partial \dot{x}}{\partial y}\right) \Delta t^{n+1 / 2} .
\end{aligned}
$$

\section{Pressure Equation of State}

$$
P_{\text {(1) }}^{n+1}=A\left(v_{(1)}^{n+1}\right)+B\left(v_{\text {(1) }}^{n+1}\right) E_{\text {(1) }}^{n+1} \text {, }
$$

where $A$ and $B$ are functions of the volume $V$ and $E$ is the internal energy.

\section{Tutal Stresses}

$$
\begin{aligned}
& \left(\Sigma_{x x}\right)_{(1)}^{n+1}=-\left(P_{(1)}^{n+1}+q_{(1)}^{n+1 / 2}\right)+\left(s_{x x}\right)_{(1)}^{n+1}, \\
& \left(\Sigma_{y y}\right)_{(1)}^{n+1}=-\left(P_{(1)}^{n+1}+q_{(1)}^{n+1 / 2}\right)+\left(s_{y y}\right)_{(1)}^{n+1}, \\
& \left(\Sigma_{z z}\right)_{(1)}^{n+1}=-\left(\text { (1) }_{(1)}^{n+1}+q_{(1)}^{n+1 / 2}\right)+\left(s_{z z}\right)_{(1)}^{n+1}
\end{aligned}
$$

von Mises Yield Condition

$$
\begin{aligned}
& { }_{(1)}^{n+1}=\left\{\left(\left(s_{x x}\right)^{2}+\left(s_{y y}\right)^{2}+\left(s_{z z}\right)^{2}\right]+2\left[\left(T_{x y}\right)^{2}+\left(T_{y z}\right)^{2}+\left(T_{z x}\right)^{2}\right]\right\}_{(1)}^{n+1} . \\
& K_{(1)}^{n+1}=2 J_{(1)}^{n+1}-\frac{2}{3}\left(Y_{(1)}^{n}\right)^{2} .
\end{aligned}
$$


If: $K_{(1)}^{n+1} \leqslant 0$ use the stress deviaturs as defined above.

If: $k_{1}^{n+1}>0$, then multiply each of the stresses $\left(s_{x x}\right)_{(1)}^{n+1} \cdot\left(s_{y y}\right)_{(1)}^{n+1} \cdot\left(s_{z z}\right)_{(1)}^{n+1},\left(T_{x y}\right)_{(1)}^{n+1} \cdot\left(T_{y z}\right)_{(1)}^{n+1}$ and $\left(T_{\mathrm{zx}}\right)_{(1)}^{\mathrm{n}+1}$ by $\sqrt{2 / 3} \mathrm{Y}_{(1)}^{\mathrm{n}} \sqrt{2 \mathrm{~J}_{(1)}^{\mathrm{n}+1}}$.

Principal Stress Deviators (for Edit Routine)

$$
\begin{aligned}
& s_{1}=2 \sqrt{-a / 3} \cos \phi / 3, \\
& s_{2}=2 \sqrt{-a / 3} \cos (\phi / 3+2 \pi / 3), \\
& s_{3}=2 \sqrt{-3 / 3} \cos (\phi / 3+4 \pi / 3) ;
\end{aligned}
$$

where

$$
\begin{aligned}
& a=\left[\left(s_{y y} s_{z z}+s_{x x} s_{y y}+s_{x x} s_{z z}\right)-\left(T_{y z}^{2}+T_{x y}^{2}+T_{z x}^{2}\right)\right], \\
& b=\left[-s_{x x} s_{y y} s_{z z}+s_{x x} T_{y z}^{2}+s_{z z} T_{x y}^{2}+s_{y y} T_{z x}^{2}-2 T_{y z} T_{x y} T_{z x}\right], \\
& \phi=\cos ^{-1}\left[ \pm\left(\sqrt{\frac{27}{-a}}\right)\left(\frac{b}{2 a}\right)\right] . \text { Take the plus sign for } b<0 \text { and minus sign for } b>0 .
\end{aligned}
$$

\section{Artificial Viscosity}

An artificial viscosity is required to permit shocks to form in the grid. The artificial viscosity, q, used here is composed of a quadratic term and a linear term in the rate of change of volume. The quadratic portion is identical to the von Neumann $q$ for calculating shocks. ${ }^{4}$ The linear term provides damping for oscillations that occur behind the shock with the $q$ method of calculations.

$$
\begin{aligned}
& \text { qu }^{n+1 / 2}=\left(\frac{\rho^{0}}{v^{n+1 / 2}}\right)\left[C_{A}^{2} l^{2}\left(\frac{\dot{V}}{V}\right)^{2}+C_{L} l a\left(\frac{\dot{V}}{V}\right)\right]_{(1)}^{n+1 / 2} \text { for } \frac{\dot{V}}{V}<0 ; q=0 \text { for } \frac{\dot{v}}{V} \geqslant 0 \\
& e_{(1)}^{\mathrm{n}+1 / 2}=\sqrt[3]{v_{(1)}^{\mathrm{n}+1 / 2}} ; v_{(1)}^{\mathrm{n}+1 / 2}=\frac{1}{2}\left(v_{(1)}^{\mathrm{n}+1}+v_{(1)}^{\mathrm{n}}\right) \\
& \stackrel{\mathrm{a}}{\mathrm{a}}=\left(\sqrt{\frac{\mathrm{P}^{\mathrm{n}} \mathrm{V}^{\mathrm{n}}}{\rho^{0}}}\right)_{(1)}
\end{aligned}
$$




$$
\begin{aligned}
& \left(\frac{\dot{v}}{v}\right)_{(1)}^{n+1 / 2}=\left(\frac{\Delta v}{v}\right)^{n+1 / 2} \frac{1}{\Delta t^{n+1 / 2}} \\
& C_{A} \cong 2 ; c_{L} \cong 0.8
\end{aligned}
$$

The $\mathrm{q}^{\mathrm{n}+1 / 2}$ is added to the pressure, $\mathrm{P}^{\mathrm{n}+1}$.

\section{Tensor Artificial Viscosity for Stabilizing the Grid}

For quasi-static problems in solid mechanies nonphysical numerical oscillations can occur in the grid under certain boundary conditions. A tensor viscosity based on the rate of strain of volume elements formed by the zone comers is used to damp this type oscillation. Referring to Fig. B-2 it is seen that surrounding point 0 there are eight tetrahedrons defined by the corners of the eight zones. A Navier-Stoke type tensor viscosity based on the rates of strain of the tetrahedron volumes is calculated for each tetrahedron that contains $\mathbf{0}$, Fig. B-2. The details for calcolating the components of viscosity for the tetrahedron in zone (1) are given below.

The tetrahedron corresponding to zone (1) is shown in Fig. B-3. The grid numbering follows the scheme shown in Fig. B-1. Here grid point 1 cerresponds to point 0 of Fig. B-2. The finite difference integration mapping proceciure is applied to the four surfaces of the tetrahedron formed by vectors, $\vec{A}, \vec{B}, \vec{C}$, of Fig. B-3.

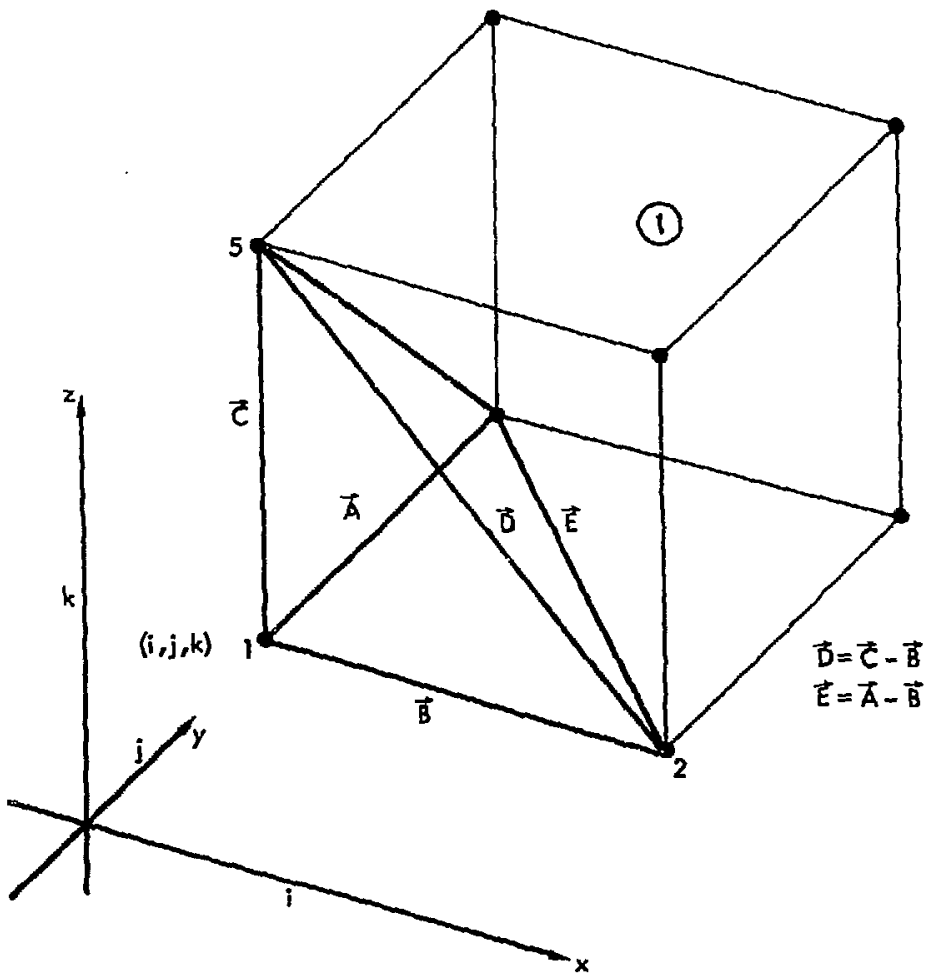

Fig. B-3. Grid numbering scheme for calculating the tensor viscosity of the tetrahedron associater with point $(i, j, k)$ of Zone (1). 
Volume " $\mathrm{ABC}$ formed by the vectors $\vec{A}, \vec{B}, \vec{C}$ of Fig. $B-3$ is:

$$
\left(v_{A B C}\right)^{n+1}=\frac{1}{6}(\vec{B} \times \vec{A}) \vec{C}=\frac{1}{6}\left[b_{j}\left(a_{j} c_{k}-a_{k} c_{j}\right)-b_{j}\left(a_{i} c_{k}-a_{k} c_{j}\right)+b_{k}\left(a_{j} c_{j}-a_{j} c_{i}\right]^{n+1}\right.
$$

The notation for the components of the vectors is the same as used for the vectors of the volume of zone(1).

\section{Velocity Derivatives}

Velocity derivatives cerresponding to the tetrahedron, Fig. B-3.

$$
\left(\frac{\partial \dot{x}}{\partial x}\right)^{n+1 / 2}=\left(\frac{1}{6 \nu_{A B C}^{n+1 / 2}}\right)\left[\dot{x}_{A B}(\vec{A} \times \vec{B}) \cdot \vec{i}+\dot{x}_{C A}(\vec{C} \times \vec{A}) \cdot \vec{i}+\dot{x}_{B C}(\vec{B} \times \vec{C}) \cdot \dot{i}+\dot{x}_{E D}(\vec{E} \times \vec{D}) \cdot \vec{i}\right]^{n+1 / 2}
$$

where

$$
\dot{x}_{A B}=\left(\dot{x}_{1}+\dot{x}_{2}+\dot{x}_{4}\right), \dot{x}_{C A}=\left(\dot{x}_{1}+\dot{x}_{4}+\dot{x}_{5}\right), \dot{x}_{B C}=\left(\dot{x}_{1}+\dot{x}_{2}+\dot{x}_{5}\right), \dot{x}_{E D}=\left(\dot{x}_{2}+\dot{x}_{4}+\dot{x}_{5}\right),
$$

and $v_{A B C}^{n+1 / 2}=\frac{1}{2}\left[v_{A B C}^{n}+\nu_{A B C}^{n+1}\right]$.

This expression can be simplified by expressing vectors $\vec{D}$ and $\vec{E}$ in terms of vectors $\vec{A}$ and $\vec{B}$.

$$
\left(\frac{\partial \dot{x}}{\partial x}\right)^{n+1 / 2}=\left(\frac{1}{6 \nu_{A B C}^{n+1 / 2}}\right)\left[\left(\dot{x}_{1}-\dot{x}_{5}\right)(\vec{A} \times \vec{B}) \cdot \vec{i}+\left(\dot{x}_{1}-\dot{x}_{2}\right)(\vec{C} \times \vec{A}) \cdot \vec{i}+\left(\dot{x}_{1}-\dot{x}_{4}\right)(\vec{B} \times \vec{C}) \cdot \vec{i}\right]^{n+1 / 2}
$$

where

$$
\begin{aligned}
& \left.(\vec{A} \times \vec{B} \cdot \vec{i})=\left[a_{j}\left(a_{j} b_{k}-a_{k} b_{j}\right)\right], \overrightarrow{(C} \times \vec{A} \cdot \vec{j}\right)=\left[c_{i}\left(c_{j} a_{k}-c_{k} a_{j}\right)\right] \text {, and }(\vec{B} \times \vec{C} \cdot \vec{i})=\left[b_{i}\left(b_{j} c_{k}-b_{k} c_{j}\right)\right\} . \\
& \frac{\partial \dot{x}}{\partial y}=\left(\frac{1}{6 v_{A B C}^{n+1 / 2}}\right)\left[\left(\dot{x}_{1}-\dot{x}_{5}\right)(\vec{A} \times \vec{B}) \cdot \vec{j}+\left(\dot{x}_{1}-\dot{x}_{2}\right)(\vec{C} \times \vec{A}) \cdot \vec{j}+\left(\dot{x}_{1}-\dot{x}_{4}\right)(\vec{B} \times \vec{C}) \cdot \vec{j}\right]^{n+1 / 2},
\end{aligned}
$$

where

$$
\begin{aligned}
& \vec{A} \times \vec{B} \cdot \vec{j}=\left[-a_{j}\left(a_{i} b_{k}-a_{k} b_{i}\right)\right], \vec{C} \times \vec{A} \cdot \vec{j}=\left[-c_{j}\left(c_{i} a_{k}-c_{k} a_{j}\right) l_{2} \text { and }(\vec{B} \times \vec{C} \cdot \vec{j})=\left[-b_{j}\left(b_{i} c_{j}-b_{k} c_{j}\right)\right] .\right. \\
& \frac{\partial \dot{x}}{\partial z}=\left(\frac{1}{6 v_{A B C}^{n+1 / 2}}\right)\left[\left(\dot{x}_{1}-\dot{x}_{5}\right)(\vec{A} \times \vec{B}) \cdot \vec{k}+\left(\dot{x}_{1}-\dot{x}_{2}\right)(\vec{C} \times \vec{A}) \cdot \vec{k}+\left(\dot{x}_{1}-x_{4}\right)(\vec{B} \times \vec{C}) \cdot \vec{k}\right]^{n+1 / 2}
\end{aligned}
$$

where

$\vec{A} \times \vec{B} \cdot \vec{k}=\left[a_{k}\left(a_{i} b_{j}-a_{j} b_{i}\right)\right], \vec{C} \times \vec{A} \cdot \vec{k}=\left[c_{k}\left(c_{i} a_{j}-c_{j} a_{i}\right)\right], \vec{B} \times \vec{C} \cdot \vec{k}=\left[b_{k}\left(b_{i} c_{j}-b_{j} c_{i}\right)\right]$. 
$\frac{\partial \dot{y}}{\partial x}$ and $\frac{\partial \dot{z}}{\partial x}$ are calculated in the same way as $\partial \dot{x} / \partial x$, but replace $\dot{x}$ by $\dot{y}$ and then $\dot{z}$.

$\frac{\partial \dot{y}}{\partial y}$ and $\frac{\partial \dot{z}}{\partial y}$ are calculated in the same way as $\partial \dot{x} / \partial y$, but replace $\dot{x}$ by $\dot{y}$ and then $\dot{z}$.

$\frac{\partial \dot{y}}{\partial z}$ and $\frac{\partial \dot{z}}{\partial z}$ are calculated in the same way as $\partial \dot{x} / \partial z$, but replace $\dot{x}$ by $\dot{y}$ and then $\dot{z}$

\section{Rates of Strain}

Components of the rate of strain of the tetrahedron defined by vectors A, B, C, Fig. B-3 are

$\dot{\epsilon}_{\mathrm{xx}}=\frac{\partial \dot{x}}{\partial x}, \dot{\epsilon}_{\mathrm{yy}}=\frac{\partial \dot{y}}{\partial y}, \dot{\epsilon}_{z z}=\frac{\partial \dot{z}}{\partial z}, \dot{\epsilon}_{x y}=\left(\frac{\partial \dot{x}}{\partial y}+\frac{\partial \dot{y}}{\partial x}\right), \dot{\epsilon}_{y z}=\left(\frac{\partial \dot{y}}{\partial z}+\frac{\partial \dot{z}}{\partial y}\right), \dot{\epsilon}_{z x}=\left(\frac{\partial \dot{x}}{\partial z}+\frac{\partial \dot{z}}{\partial x}\right), \frac{\dot{v}}{v}=\frac{\partial \dot{x}}{\partial x}+\frac{\partial \dot{y}}{\partial y}+\frac{\partial \dot{z}}{\partial z}$.

\section{Artificial Viscosity}

Tensor artificial viscosity for tetrahedron A, B, C, Fig. B-3, is

$$
\begin{aligned}
\mathrm{q}_{\mathrm{xx}}^{\mathrm{n}+1 / 2}=2 \mu_{1}\left[\dot{\epsilon}_{\mathrm{xx}}-\frac{1}{3} \frac{\dot{v}}{\mathrm{v}}\right]^{\mathrm{n}+1 / 2}, \mathrm{q}_{\mathrm{yy}}^{\mathrm{n}+1 / 2}=2 \mu_{1}\left[\dot{\epsilon}_{\mathrm{yy}}-\frac{1}{3} \frac{\dot{v}}{\mathrm{v}}\right]^{\mathrm{n}+1 / 2}, \mathrm{q}_{\mathrm{zz}}^{\mathrm{n}+1 / 2}=2 \mu_{1}\left[\dot{\epsilon}_{\mathrm{zz}}-\frac{1}{3} \frac{\dot{v}}{\mathrm{v}}\right]^{\mathrm{n}+1 / 2}, \\
\mathrm{q}_{\mathrm{xy}}^{\mathrm{n}+1 / 2}=\mu_{1}\left[\dot{\epsilon}_{\mathrm{xy}}\right]^{\mathrm{n}+1 / 2}, \mathrm{q}_{\mathrm{yz}}=\mu_{1}\left[\dot{\epsilon}_{\mathrm{yz}}\right]^{\mathrm{n}+1 / 2}, \mathrm{q}_{\mathrm{zx}}=\mu_{1}\left[\dot{\epsilon}_{\mathrm{zx}}\right]^{\mathrm{n}+1 / 2}, \\
\text { where } \mu_{1}=\left[\mathrm{C}_{\mathrm{NS}}\left(\frac{\rho^{0}}{\mathrm{v}}\right)_{(1)} \sqrt{1}^{\nu_{\mathrm{ABC}}}\right]^{\mathrm{n}+1}, \\
\mathrm{C}_{\mathrm{NS}}=\text { constant } \approx 10^{-2}, \\
\rho^{0}=\text { reference density of zone (1). } \\
\mathrm{v}=\text { relative volume of zone (1). }
\end{aligned}
$$

The above components of the tensor artificial viscosity are added to the corresponding components of the stress tensor defined at time $n+1$.

\section{Energy Equation}

\section{Distortion Energy Increment}

$$
\Delta z_{(1)}^{n+1 / 2}=V_{(1)}^{n+1 / 2}\left[s_{x x} \Delta \varepsilon_{x x}+s_{y y} \Delta \varepsilon_{y y}+s_{z z} \Delta \varepsilon_{z z}+T_{x y} \Delta \varepsilon_{x y}+T_{y z} \Delta \varepsilon_{y z}+T_{z x} \Delta \varepsilon_{z x}\right]_{(1)}^{n+1 / 2} \text {. }
$$


Total Internal Energy per Original Volume

$$
\begin{aligned}
E^{n+1} & =\left(\frac{E^{n}-\left\{\frac{1}{2}\left[A\left(V^{n+1}\right)+P^{n}\right]+\bar{q}\right\} \cdot\left(V^{n+1}-V^{n}\right)+\Delta z^{n+1 / 2}}{1+\frac{1}{2}\left[B\left(V^{n+1}\right)\right] \cdot\left(V^{n+1}-V^{n}\right)}\right)_{(1)} \\
\bar{q} & =\frac{1}{2}\left(q^{n+1 / 2}+q^{n-1 / 2}\right)
\end{aligned}
$$

\section{Plastic Strain}

In the following definitions of plastic strain the stress deviators at time $(n+1)$ are taken as the values after the yield condition has been satisfied.

\section{Components of Plastic Strain Rate}

$$
\begin{aligned}
& \mathrm{P}_{\mathbf{x x}}^{n+1 / 2}=\dot{\epsilon}_{\mathrm{xx}}^{\mathrm{n+1} / 2}-\frac{1}{\Delta \mathrm{t}^{\mathrm{n}+1 / 2}}\left[\frac{\mathrm{s}_{\mathrm{xx}}^{\mathrm{n}+1}-\mathrm{s}_{\mathrm{xx}}^{\mathrm{n}}}{2 \mu}+\frac{1}{3} \frac{\mathrm{V}^{\mathrm{n}+1}-\mathrm{V}^{\mathrm{n}}}{\mathrm{v}^{\mathrm{n}+1 / 2}}\right], \\
& \mathrm{P}_{\epsilon_{\mathrm{yy}}^{\mathrm{n}}}^{\mathrm{n}+1 / 2}=\epsilon_{\mathrm{yy}}^{\mathrm{n+1} / 2}-\frac{1}{\Delta \mathrm{t}^{\mathrm{n}+1 / 2}}\left[\frac{\mathrm{s}_{\mathrm{yy}}^{\mathrm{n}+1}-\mathrm{s}_{\mathrm{yy}}^{\mathrm{n}}}{2 \mu}+\frac{1}{3} \frac{v^{\mathrm{n}+1}-\mathrm{V}^{\mathrm{n}}}{\mathrm{v}^{\mathrm{n}+1 / 2}}\right] \text {, } \\
& \mathrm{p}_{\dot{E}_{\mathrm{zZ}}^{n+1 / 2}}^{\mathrm{n}}=\dot{\epsilon}_{\mathrm{zZ}}^{\mathrm{n}+1 / 2}-\frac{1}{\Delta \mathrm{t}^{\mathrm{n}+1 / 2}}\left[\frac{\mathrm{s}_{\mathrm{zz}}^{\mathrm{n}+1}-\mathrm{s}_{\mathrm{zz}}^{\mathrm{n}}}{2 \mu}+\frac{1}{3} \frac{\mathrm{v}^{\mathrm{n}+1}-\mathrm{v}^{\mathrm{n}}}{\mathrm{v}^{\mathrm{n}+1 / 2}}\right] \text {, } \\
& \mathrm{p}_{\dot{\epsilon}_{x y}^{n+1 / 2}}^{n+1}=\dot{\epsilon}_{x y}^{n+1 / 2}-\frac{1}{\Delta t^{n+1 / 2}}\left[\frac{\mathrm{T}_{x y}^{n+1}-T_{x y}^{n}}{\mu}\right] \text {, } \\
& \mathrm{p}_{\mathrm{zx}}^{\mathrm{n}+1 / 2}=\dot{\epsilon}_{\mathrm{zx}}^{\mathrm{n}+1 / 2}-\frac{1}{\Delta \mathrm{t}^{\mathrm{n}+1 / 2}}\left[\frac{\mathrm{T}_{\mathrm{zx}}^{\mathrm{n}+1}-\mathrm{T}_{\mathrm{zx}}^{\mathrm{n}}}{\mu}\right] \text {, } \\
& \mathrm{p}_{\epsilon_{\mathrm{yz}}}^{\mathrm{n}+1 / 2}=\dot{\epsilon}_{\mathrm{yz}}^{\mathrm{n+1} / 2}-\frac{1}{\Delta \mathrm{t}^{\mathrm{n}+1 / 2}}\left[\frac{\mathrm{T}_{\mathrm{yz}}^{\mathrm{n}+1}-\mathrm{T}_{\mathrm{yz}}^{\mathrm{n}}}{\mu}\right] \text {. }
\end{aligned}
$$

\section{Equivalent Plastic Strain}

$$
\begin{aligned}
\left(\dot{\epsilon}^{\mathrm{P}}\right)^{\mathrm{n}+1 / 2}= & \frac{\sqrt{2}}{3}\left\{\left(\mathrm{P}_{\dot{\epsilon}_{\mathrm{xx}}}-\mathrm{P}_{\dot{\epsilon}_{\mathrm{yy}}}\right)^{2}+\left(\mathrm{P}_{\dot{\epsilon}_{\mathrm{yy}}}-\mathrm{P}_{\dot{\epsilon}_{\mathrm{zz}}}\right)^{2}\right. \\
& +\left({ }^{\mathrm{P}} \dot{\epsilon}_{\mathrm{zz}}-\mathrm{P}_{\dot{\epsilon}_{\mathrm{xx}}}\right)^{2} \\
& \left.+\frac{3}{2}\left[\left({ }^{\mathrm{P}} \dot{\epsilon}_{\mathrm{xy}}\right)^{2}+\left(\mathrm{P}_{\dot{\epsilon}_{\mathrm{zx}}}\right)^{2}+\left(\mathrm{P}_{\dot{\epsilon}_{\mathrm{yz}}}\right)^{2}\right]\right\}^{1 / 2},
\end{aligned}
$$




$$
\left(\bar{\epsilon}^{p}\right)^{n+1}=\left(\bar{E}^{p}\right)^{n}+\left(\dot{\epsilon}^{p}\right)^{n+1 / 2} \Delta t^{n+1 / 2} .
$$

\section{Flow Stress}

$$
Y^{n+1}=a\left[b+\left(\bar{e}^{p}\right)^{n+1}\right]^{c}
$$

Here $a, b$ and $c$ are material constants, not to be confused with the vector components $a_{i, j}, k$ etc.

\section{Time Step}

$$
\left(\Delta_{i}\right)^{n+3 / 2}=\left.0.9 \frac{L^{n+1}}{\sqrt{a^{2}+b^{2}}}\right|_{\text {Min. of all zones }}
$$

and $(\Delta t)^{n+3 / 2} \leqslant 1.1 \Delta t^{n+1 / 2}$.

$L$ is the minimum zone thickness, defined as

$$
L^{n+1}=\frac{v^{n+1}}{S_{m}^{n+1}} \text {, where } \nu^{n+1}=\text { volume of zone associated with point } i, j, k \text { at } t^{n+1} \text {, and } S_{m}^{n+1} \text { is the }
$$

maximum of $(\bar{A})^{2},(\bar{B})^{2}$ and $(\bar{C})^{2}$. Here $\bar{A}, \bar{B}$, and $\bar{C}$ are the lengths of vectors $A, B, C$ at time $t^{n+1}$.

$$
\left(\overline{\mathrm{A}}_{1}\right)^{2}=\left(a_{i}^{2}+a_{j}^{2}+a_{k}^{2}\right)_{1},\left(\vec{B}_{1}\right)^{2}=\left(b_{i}^{2}+b_{j}^{2}+b_{k}^{2}\right)_{l} \cdot\left(\bar{C}_{1}\right)^{2}=\left(c_{i}^{2}+c_{j}^{2}+c_{k}^{2}\right)_{l} .
$$

See Fig. B-1 et seq. for this vector notation. Also, in this equation for $\Delta t$,

$$
\begin{aligned}
& a=\text { sound speec calculated from the equation of state and } \\
& b=8\left[C_{A}^{2}+C_{L}\right] L^{n+1}\left(\frac{\Delta V}{V} \cdot \frac{1}{\Delta t}\right)^{n+1 / 2}
\end{aligned}
$$

where $C_{A}$ and $C_{L}$ are the quadratic and the linear q constants, respectively.

Further, $(\Delta t)^{n+1}=\frac{1}{2}\left(\Delta t^{n+3 / 2}+\Delta t^{n+1 / 2}\right)$.

\section{Boundary Conditions}

Pseudo zones with zero mass are assumed to surround the grid that defines the physical cbject. Thus points assaciated with the surface of the physical object may be calculated without changing the logic. Nornally a free surface boundary condition is provided, i.e. the pseudo zone pressures are considered always equal to zero. Pressure boundary conditions may be applied by entering the desired space-time values into the pseudo zones.

A reflection boundary condition is obtained by setting equal to zero the normal component of accelerations of a surface point when it points into the reflection surface. 


\section{CHECK PROBLEMS AND APPLICATIONS}

\section{Simple Harmonic Motion}

The calculation of the motion of a vibrating plate, clamped at one end, provides a problem that can be readily checked by elasticity theory. Orienting the plate at an arbitrary angle in three dimensional space activates all six components of the stress tensor.

In the calculations shown in Fig. B-4 an elastic plate clamped at the top is set into motion by applying a velocity $v=10 \mathrm{~m} / \mathrm{s}$ to the lower right edge in the direction perpendicular to the edge for a time $t=50 \mu \mathrm{s}$. After this time the applied velocity is released, but the lower portion of the plate continues to move due to the kinetic erecgy. Actually upon telease the end of the plate initially moves faster than the applied velocity since this velocity does not correspond to the natural frequency of the plate. Figure B-4d is a time-displacement plo, for a position in the geometric center of the bottom plane of the plate. It is easily verified that the calculation reproduces the fundamental frequency of the plate.
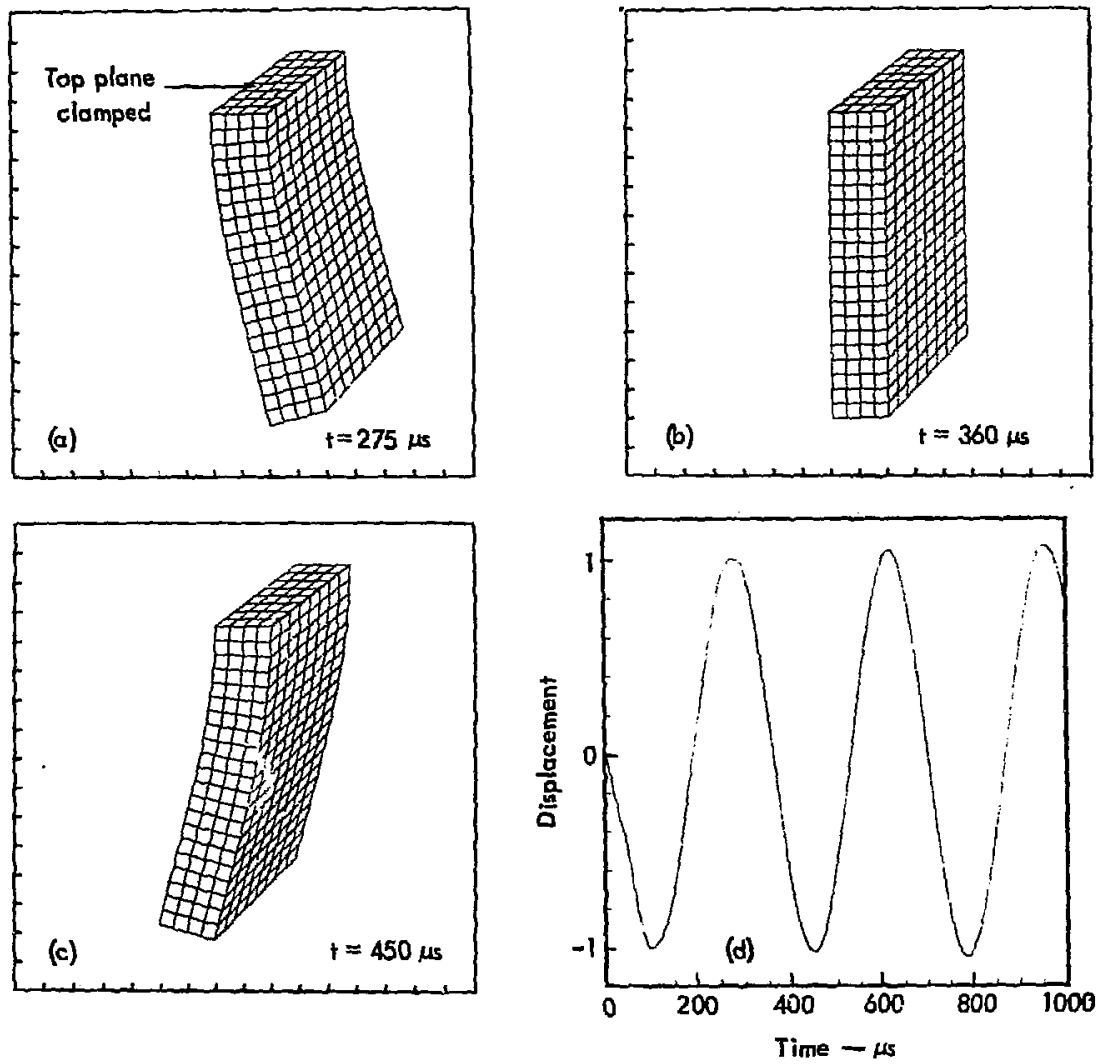

Fig. B-4. Simulation of the motion of a vibrating elastic plate. (a) Position of maximum positive displacement, $t=275 \mu \mathrm{s}$. (b) Position of maximum kinetic energy, $t=360 \mu$ s. (c) Position of maximum negative displacement, $t=450 \mu$ s. (d) Displacement history for a point in the geometric center of the bottom plane. 


$$
\begin{aligned}
& \text { Dimensions } \begin{cases}\text { length: } & \mathrm{L}=52.5 \mathrm{~mm} \\
\text { width: } & \mathrm{W}=20.0 \mathrm{~mm} \\
\text { thickness: } T=10.0 \mathrm{~mm}\end{cases} \\
& \text { Elastic constants: } \begin{array}{l}
\text { bulk modulus: } \quad \mathrm{k}=188 \mathrm{GPa} \\
\text { shear modulus: } \mu=81.4 \mathrm{GPa} \\
\text { density: } \quad \rho 0=7.72 \mathrm{Mg} / \mathrm{m}^{3}
\end{array}
\end{aligned}
$$

The tensor artificial viscosity used in this calculation is $C_{N S}=0.05$, more than enough to suppress grid oscillations that would otherwise occur. Figure B-4d shows that the amplitude of the oscillation has not been damped or affected by the artificial viscosity.

\section{Plasticity}

The impact of a right circular cylinder on a rigid boundary provides a calculation to test the plasticity aspect of the computer program. Since this problem requires only two space dimension it can be calculated with the HEMP code. ${ }^{1}$ Figure B-5a shows results of the HEMP calculation where cylindrical symmetry is incorporated into the fundamental equations. Figure B-Sb shows results of the same problem calculated with the HEMP 3D program described here. It can be seen in Fig. B-5b that the cylinder has been discretized with three dimensional zones. The calculated time to stop the cylinder, $30 \mu \mathrm{s}$, and the final cylinder length, $19.28 \mathrm{~mm}$, were the same for both HEMP and HEMP 3D. Comparison of the cylinder profiles at $t \approx 30 \mu$ also showed almost identical results.

\section{Fracture Mechanics}

Figure B-6 shows an application of the code to a problem in fracture mechanics. An elastic material with an eliptical suriace crack is stressed in tension, perpendicular to the crack surface. Figure B-6 shows the calculated stress contours around the crack. 

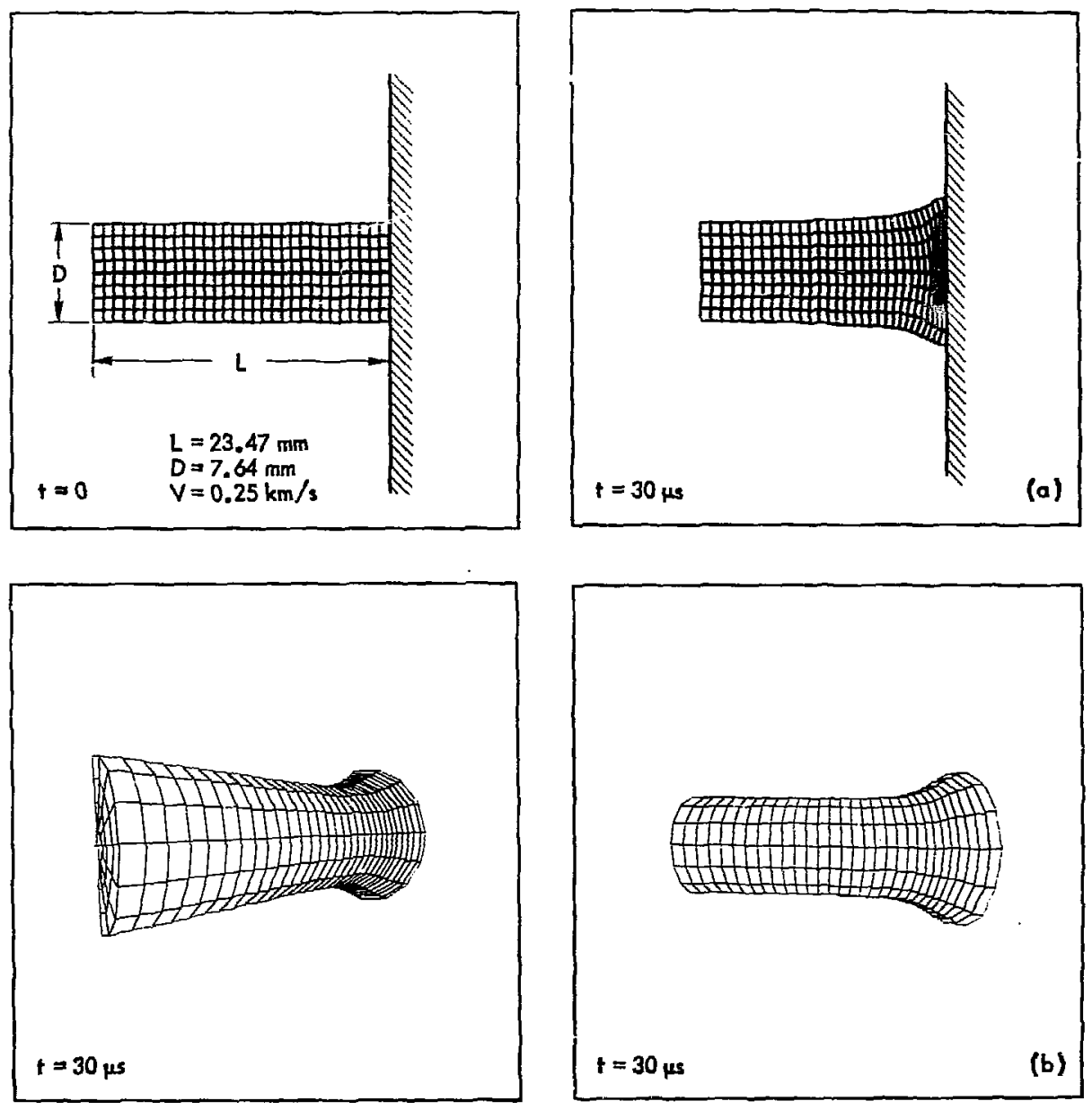

Fig. B-5. Sinulatioli of the impact of a cylinder on a rigid wall.

Constitutive model:

$$
\begin{array}{ll}
\text { Pressure } & P=76\left(\frac{\rho}{\rho^{0}}-1\right) \\
\text { Density } & \rho^{0}=2.7 \mathrm{Mg} / \mathrm{m}^{3} \\
\text { Shear moduius } & \mu=24.8 \mathrm{GPa} \\
\text { Flow stress } & \mathrm{Y}=0.46\left(0.008+\epsilon^{\mathrm{P}}\right)^{0.1} \mathrm{GPa}
\end{array}
$$

$\epsilon^{\mathbf{P}}$ is the equivalent plastic strain.

(a) Before and after views using the two-dimensional HEMP code.

(b) Before and after views using the HEMP 3D code. 

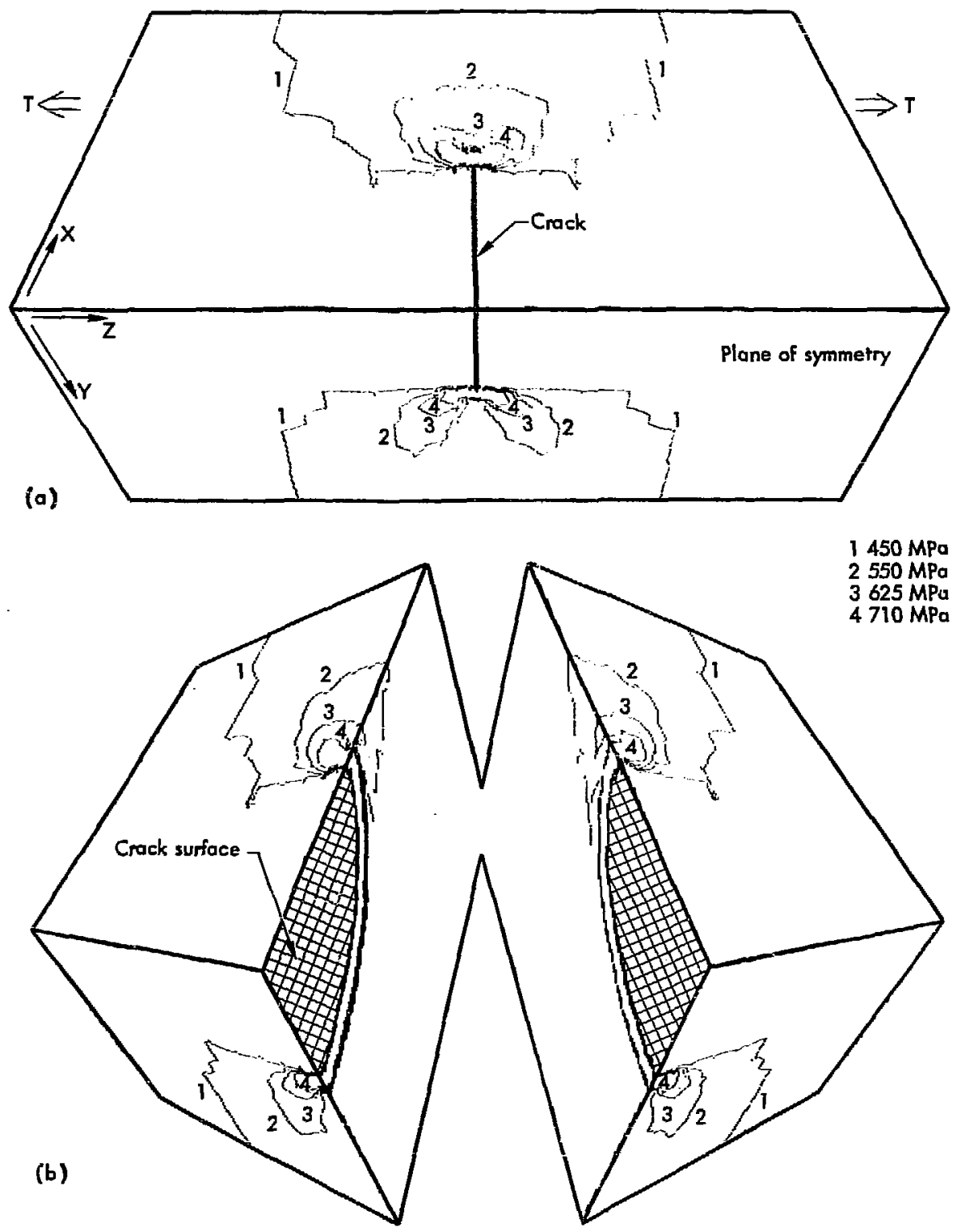

Fig. B-6. Calculation of the stress field around an elliptical surface crack in an elastic-plastic solid. Density, $\rho 0=7.90 \mathrm{Mg} / \mathrm{m}^{3}$; bulk modulus, $\mathrm{k}=165 \mathrm{GPa}$; sh .ar modulus, $\mu=165 \mathrm{GPa}$. 


\section{Appendix C \\ Comparison of the Finite Difference and Finite Element \\ Methods for the Solution of Poisson's Equation}

\section{INTRODUCTION}

The finite difference approximation discussed in Ref. 1 and the isoparametric finite element (with one-point integration), Ref. 5, are used to obtain difference equations for Poisson's equation. The resuiting difference equations are compared. Some general comments relating to the plane elasticity problem are also made.

\section{Differential Equation and Boundary Conditions}
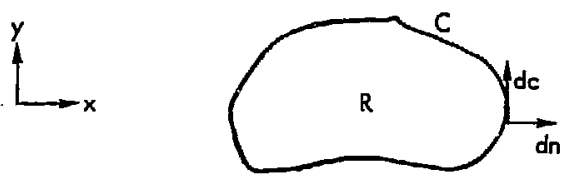

$\frac{\partial^{2} \phi}{\partial x^{2}}+\frac{\partial^{2} \phi}{\partial y^{2}}+f(x, y)=0$ in $R$

with $\phi+\mathrm{h}_{2}=0$ on $\mathrm{C}_{1}$ and $\frac{\partial \phi}{\partial \mathrm{n}}+\mathrm{g}=0$ on $\mathrm{C}_{2}\left(\mathrm{C}_{1} \cup \mathrm{C}_{2}=\mathrm{C}\right)$

Equations (C-1) and (C-2) are equivalent to the variational equation

$$
\delta\left\{\int_{\mathbf{R}} \frac{1}{2}\left[-\left(\frac{\partial \phi}{\partial x}\right)^{2}-\left(\frac{\partial \phi}{\partial y}\right)^{2}+2 f \phi\right] d x d y-\int_{C_{2}} g \phi d C_{2}\right\}=0
$$

with

$$
\phi+h=0 \text { on } C_{1} \text {. }
$$

\section{FINITE DIFFERENCE EQUATIONS}

For a general quadrilateral grid (see Ref. 1, pp. 35-38) the first partial derivatives can be expressed in terms of contour integrals as follows:

$$
\iint_{R} \frac{\partial \phi}{\partial x} d x d y=\int_{C} \phi d y
$$


and

$$
\iint_{R} \frac{\partial \phi}{\partial y} d x d y=-\int_{C} \phi d x,
$$

where $\mathrm{R}$ is a simply connected region bounded by $\mathrm{C}$. In the limit

$$
\frac{\partial \phi}{\partial x}=\lim A \rightarrow 0 \frac{\int^{C} \phi d y}{A}
$$

and

$$
\frac{\partial \phi}{\partial y}=-\lim A \rightarrow 0 \frac{\int_{C} \phi d x}{A} \text {, }
$$

where $A$ is the area of $R$.

For the grid shown at the right

the first partial derivatives evaluated at $(i-1 / 2, j-1 / 2)$ can be approximated by

$$
\begin{gathered}
\left.\frac{\partial \phi}{\partial x}\right|_{i-\frac{1}{2}, j-\frac{1}{2}}=\frac{1}{A_{i}}-\frac{1}{2}, j-\frac{1}{2}\left[\int_{i, j}^{i-1, j} \phi d y+\int_{i-1, j}^{i-1, j-1} \phi d y\right. \\
\left.+\int_{i-1, j-1}^{i, j-1} \phi d y+\int_{i, j-1}^{i, j} \phi d y\right],
\end{gathered}
$$
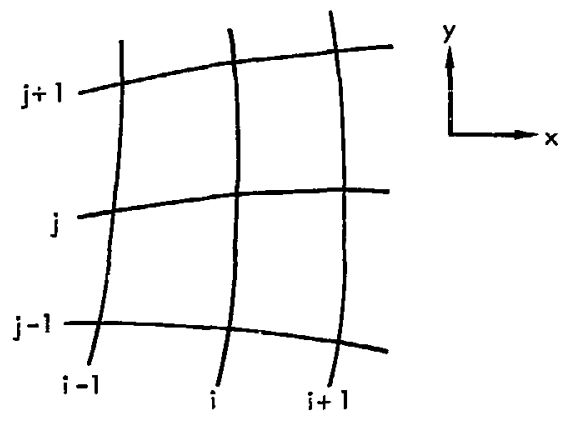

where $A_{i-1 / 2, j-1 / 2}=$ area bounded by the $j, j-1, i$ and $i-i$.

Letting

$$
\begin{aligned}
\phi & =\frac{\phi_{i, j}+\phi_{i-1, j}}{2} \text { for the first integral in (C-8) } \\
& =\frac{\phi_{i-1, j}+\phi_{i-1, j-1}}{2} \text { for the second integral, } \\
& =\frac{\phi_{i-I, j-1}+\phi_{i, j-1}}{2} \text { for the third integral, and }
\end{aligned}
$$




$$
=\frac{\phi_{i, j-1}+\phi_{i, j}}{2} \text { for ine fourth integral }
$$

and integrating yields:

$\left.\frac{\partial \phi}{\partial x}\right|_{i-\frac{1}{2}, j-\frac{1}{2}}=\frac{1}{2 A_{i}}-\frac{1}{2}, j-\frac{1}{2}\left[\left(\phi_{i, j-1}-\phi_{i-1, j}\right)\left(y_{i, j}-y_{i-1, j-1}\right)-\left(y_{i, j-1}-y_{i-1, j}\right)\left(\phi_{i, j}-\phi_{i-1, j-1}\right)\right] .(C-9)$

Similarily

$\left.\frac{\partial \phi}{\partial y}\right|_{i-\frac{1}{2}, j-\frac{1}{2}}=-\frac{1}{2 A_{i}-\frac{1}{2}, j-\frac{1}{2}}\left[\left(\phi_{i, j-1}-\phi_{i-1, j}\right)\left(x_{i, j}-x_{i-1, j-1}\right)-\left(x_{i, j-1}-x_{i-1, j}\right)\left(\phi_{i, j}-\phi_{i-1, j-1}\right)\right] .(C-10)$

The second partial derivatives are given by

$$
\frac{\partial^{2} \phi}{\partial x^{2}}=\lim A \rightarrow 0 \frac{\int_{C} \frac{\partial \phi}{\partial x} d y}{A}
$$

and

$$
\frac{\partial^{2} \phi}{\partial y^{2}}=-\lim A \rightarrow 0 \frac{\int_{C} \frac{\partial \phi}{\partial y} d x}{A} .
$$

The second partial derivatives evaluated at $(i, j)$ can be approximated by

$$
\left.\frac{\partial^{2} \phi}{\partial x^{2}}\right|_{i, j}=\frac{1}{A_{i, j}}\left[\int_{i, j-1}^{i+1, j} \frac{\partial \phi}{\partial x} d y+\int_{i+1, j}^{i, j+1} \frac{\partial \phi}{\partial x} d y+\int_{i, j+1}^{i-1, j} \frac{\partial \phi}{\partial x} d y+\int_{i-1, j}^{i, j-1} \frac{\partial \phi}{\partial x} d y\right],
$$

where $A_{i, j}$ is the area of the quadrilateral formed by the corners $(i, j-1),(i+1, j),(i, j+1),(i-1, j)$, or approximately

$$
A_{i, j}=\frac{1}{2}\left(A_{i-\frac{1}{2}}, j-\frac{1}{2}+A_{i}-\frac{1}{2}, j+\frac{1}{2}+A_{i}+\frac{1}{2}, j+\frac{1}{2} \div A_{i}+\frac{1}{2}, j-\frac{1}{2}\right) .
$$

Letting

$$
\frac{\partial \phi}{\partial x}=\left.\frac{\partial \phi}{\partial x}\right|_{i+\frac{1}{2}, j-\frac{1}{2}} \text { for the lst integral, }
$$




$$
\begin{aligned}
& =\left.\frac{\partial \phi}{\partial x}\right|_{i+\frac{1}{2}, j+\frac{1}{2}} \text { for the 2nd integral, } \\
& =\left.\frac{\partial \phi}{\partial x}\right|_{i-\frac{1}{2}, j+\frac{1}{2}} \text { for the 3rd integral, } \\
& =\left.\frac{\partial \phi}{\partial x}\right|_{i-\frac{1}{2}, j-\frac{1}{2}} \text { for the 4th integral, }
\end{aligned}
$$

and integrating yields:

$$
\begin{aligned}
\left.\frac{\partial^{2} \phi}{\partial x^{2}}\right|_{i, j} & =\frac{1}{A_{i, j}}\left[\left.\frac{\partial \phi}{\partial x}\right|_{i+\frac{1}{2}, j-\frac{1}{2}}\left(y_{i+1, j}-y_{i, j-1}\right)\right. \\
& +\left.\frac{\partial \phi}{\partial x}\right|_{i+\frac{1}{2}, j+\frac{1}{2}}\left(y_{i, j+1}-y_{i+1, j}\right)+\left.\frac{\partial \phi}{\partial x}\right|_{i-\frac{1}{2}, j-\frac{1}{2}}\left(y_{\left.i-1, j-y_{i, j+1}\right)}\right. \\
& \left.+\left.\frac{\partial \phi}{\partial x}\right|_{i-\frac{1}{2}, j-\frac{1}{2}}\left(y_{i, j-1}-y_{i-1, j}\right)\right],
\end{aligned}
$$

Similarly,

$$
\begin{aligned}
\left.\frac{\partial^{2} \phi}{\partial y^{2}}\right|_{i, j} & =\frac{-1}{A_{i, j}}\left[\left.\frac{\partial \phi}{\partial y}\right|_{i+\frac{1}{2}, j-\frac{1}{2}} ^{\left(x_{i+1, j}-x_{i, j}\right)}\right. \\
& +\left.\frac{\partial \phi}{\partial y}\right|_{i+\frac{1}{2}, j+\frac{1}{2}}\left(x_{i, j+1}-x_{i+1, j}\right)+\left.\frac{\partial \phi}{\partial y}\right|_{i-\frac{1}{2}, j+\frac{1}{2}}\left(x_{i-1, j}-x_{i, j+1}\right) \\
& \left.+\left.\frac{\partial \phi}{\partial y}\right|_{i-\frac{1}{2}, j-\frac{1}{2}}\left(x_{i, j-1}-x_{i-1, j}\right)\right]
\end{aligned}
$$


Numbering the grid as shown and expanding Eqs. (C-15) and (C-16),

$$
\left(\frac{\partial^{2} \phi}{\partial x^{2}}+\frac{\partial^{2} \phi}{\partial y^{2}}+f\right)_{5}=\sum_{i=1}^{9} \frac{\alpha_{j}}{2 A_{5}} \phi_{i}+f_{S}=0,
$$

where

$$
\begin{aligned}
\alpha_{1}= & \frac{1}{A_{1}}\left[\left(y_{4}-y_{2}\right)^{2}+\left(x_{4}-x_{2}\right)^{2} I,\right. \\
\alpha_{2}=\frac{-1}{A_{1}} & {\left[\left(y_{5}-y_{1}\right)\left(y_{4}-y_{2}\right)+\left(x_{5}-x_{1}\right)\left(x_{4}-x_{2}\right)\right] } \\
& -\frac{1}{A_{1 I}}\left[\left(y_{5}-y_{3}\right)\left(y_{6}-y_{2}\right)+\left(x_{5}-x_{3}\right)\left(x_{6}-x_{2}\right)\right],
\end{aligned}
$$

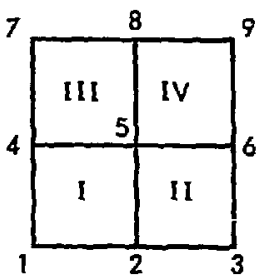

$$
\alpha_{3}=\frac{1}{A_{I I}}\left[\left(y_{6}-y_{2}\right)^{2}+\left(x_{6}-x_{2}\right)^{2}\right]
$$$$
\alpha_{4}=\frac{1}{A_{1}}\left[\left(y_{5}-y_{1}\right)\left(y_{4}-y_{2}\right)+\left(x_{5}-x_{1}\right)\left(x_{4}-x_{2}\right)\right]+\frac{1}{A_{1 I}}\left[\left(y_{7}-y_{5}\right)\left(y_{8}-y_{4}\right)+\left(x_{7}-x_{5}\right)\left(x_{8}-x_{4}\right)\right] \text {, }
$$$$
\alpha_{5}=\frac{-1}{A_{1}}\left[\left(y_{4}-y_{2}\right)^{2}+\left(x_{4}-x_{2}\right)^{2}\right]-\frac{1}{A_{I I}}\left[\left(y_{6}-y_{2}\right)^{2}+\left(x_{6}-x_{2}\right)^{2}\right]-\frac{1}{A_{I I I}}\left[\left(y_{8}-y_{4}\right)^{2}+\left(x_{8}-x_{4}\right)^{2}\right]
$$

$$
-\frac{1}{A_{I V}}\left[\left(y_{8}-y_{6}\right)^{2}+\left(x_{8}-x_{6}\right)^{2}\right]
$$

$\left.\alpha_{6}=\frac{1}{A_{1 I}} I\left(y_{5}-y_{3}\right)\left(y_{6}-y_{2}\right)+\left(x_{5}-x_{3}\right)\left(x_{6}-x_{2}\right)\right]+\frac{1}{A_{I V}}\left[\left(y_{9}-y_{5}\right)\left(y_{8}-y_{6}\right)+\left(x_{9}-x_{5}\right)\left(x_{8}-x_{2}\right)\right]$,

$\alpha_{7}=\frac{1}{\text { AIII }_{\text {II }}}\left[\left(y_{8}-y_{4}\right)^{2}+\left(x_{8}-x_{4}\right)^{2}\right]$,

$\alpha_{8}=\frac{1}{A_{I I I}}\left[\left(y_{7}-y_{5}\right)\left(y_{8}-y_{4}\right)+\left(x_{7}-x_{5}\right)\left(x_{8}-x_{4}\right)\right]-\frac{1}{A_{I V}}\left[\left(y_{9}-y_{5}\right)\left(y_{8}-y_{6}\right)+\left(x_{9}-x_{5}\right)\left(x_{8}-x_{6}\right)\right]$, and $\alpha_{9}=\frac{1}{A_{J V}}\left[\left(y_{8}-y_{6}\right)^{2}+\left(x_{8}-x_{6}\right)^{2}\right]$.

In addition to writing the field equation at all interior points the boundary conditions must be applied at all boundary points. The finite rifference boundary conditions will not be considerer here. 


\section{FINITE ELEMENT EQUATIONS}

For the general quadrilateral element shown assume a solution $(\phi)$ and iorcing function $(f)$ of the following form:

$\phi=\frac{1}{4}\left[\begin{array}{l}(1-s)(1-t) \\ (1-s)(1+t) \\ (1+s)(1+t) \\ (1+s)(1-t)\end{array}\right]^{T}\left[\begin{array}{l}\phi_{i-1, j-1} \\ \phi_{i-1, j} \\ \phi_{i, j} \\ \phi_{i, j-1}\end{array}\right]=H^{T} \Phi$

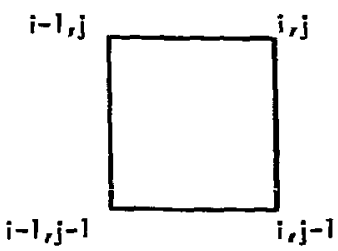

$f=H^{T} F ; F=\left[\begin{array}{l}f_{i-1, j-1} \\ f_{i-1, j} \\ f_{i, j} \\ f_{i, j-1}\end{array}\right]$

where the transformation between $(x, y)$ and $(s, t)$ is defined by

$$
x=H^{T} X, \dot{X}=\left[\begin{array}{l}
x_{i-1, j-1} \\
x_{i-1, j} \\
x_{i, j} \\
x_{i, j-1}
\end{array}\right]
$$

and

$$
y=H^{T} Y, Y=\left[\begin{array}{l}
y_{i-1, j-1} \\
y_{i-1, j} \\
y_{i, j} \\
y_{i, j-1}
\end{array}\right] \text {. }
$$

In the $s, t$ plane the contribution to the area integral of the variational equation (C-3) is

$\delta \int_{-1}^{1} \int_{-1}^{1} \frac{1}{2}\left[-\left(\frac{\partial \phi}{\partial x}\right)^{2}-\left(\frac{\partial \phi}{\partial y}\right)^{2}+2 f \phi\right]|J| d s d t$ 
where

$$
\left[\begin{array}{l}
\frac{\partial \phi}{\partial \mathrm{x}} \\
\frac{\partial \phi}{\partial y}
\end{array}\right]=[\mathrm{J}]^{-1}\left[\begin{array}{l}
\frac{\partial \phi}{\partial \mathrm{s}} \\
\frac{\partial \phi}{\partial t}
\end{array}\right]
$$

and

$$
J=\left[\begin{array}{ll}
\frac{\partial x}{\partial s} & \frac{\partial y}{\partial s} \\
\frac{\partial x}{\partial t} & \frac{\partial y}{\partial t}
\end{array}\right] .
$$

Expanding $\partial \phi / \partial \mathrm{x}$ and $\partial \phi / \partial \mathrm{y}$ yields

$$
\frac{\partial \phi}{\partial x}=\frac{1}{|\bar{J}|} \Phi^{T}\left(\frac{\partial H}{\partial s} \frac{\partial H^{T}}{\partial t}-\frac{\partial H}{\partial t} \frac{\partial H^{T}}{\partial s}\right) Y
$$

and

$$
\frac{\partial \phi}{\partial y}=\frac{-1}{|J|} \phi^{T}\left(\frac{\partial H}{\partial s} \frac{\partial H^{T}}{\partial t}-\frac{\partial H}{\partial t} \frac{\partial H^{T}}{\partial s}\right) X .
$$

A one-point approximation for the integral (C-22) yields

$$
\delta\left\{\frac{1}{2}\left[-\left(\frac{\partial \phi}{\partial x}\right)_{0,0}^{2}-\left(\frac{\partial \phi}{\partial y}\right)_{0,0}^{2}+2(f \phi)_{0,0}\right] 4|J|_{0,0} .\right.
$$

Evaluating $|J|,(\partial \phi / \partial x),(\partial \phi / \partial y)$, and $f \phi$ at $(s, t)=(0,0)$ yields

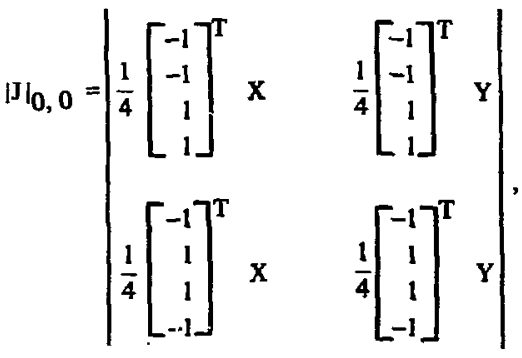

$$
\begin{aligned}
& =-\frac{1}{4} A_{i}-\frac{1}{2}, j-\frac{1}{2}
\end{aligned}
$$




$$
\begin{aligned}
& \left(\frac{\partial \phi}{\partial x}\right)_{0,0}=\frac{-4}{A_{i}-\frac{1}{2}, j-\frac{1}{2}} T^{T}\left(\frac{1}{16}\left[\begin{array}{r}
-1 \\
-1 \\
1 \\
1
\end{array}\right]\left[\begin{array}{r}
-1 \\
1 \\
1 \\
-1
\end{array}\right]^{T}-\frac{1}{16}\left[\begin{array}{r}
-1 \\
1 \\
1 \\
-1
\end{array}\right]\left[\begin{array}{r}
-1 \\
-1 \\
1 \\
1
\end{array}\right]^{T}\right) Y \\
& =\frac{-1}{2 A_{i}-\frac{1}{2}, j-\frac{1}{2}} \Phi^{T}\left[\begin{array}{rrrr}
0 & -1 & 0 & 1 \\
1 & 0 & -1 & 0 \\
0 & 1 & 0 & -1 \\
-1 & 0 & 1 & 0
\end{array}\right] Y, \\
& \left(\frac{\partial \phi}{\partial y}\right)_{0,0}=\frac{1}{2 A_{i}-\frac{1}{2}, j-\frac{1}{2}} \Phi^{T}\left[\begin{array}{rrrr}
0 & -1 & 0 & 1 \\
1 & 0 & -1 & 0 \\
0 & 1 & 0 & -1 \\
-1 & 0 & 1 & 0
\end{array}\right] X,
\end{aligned}
$$

and

$$
(\delta)_{0,0}=\frac{1}{16} \mathrm{~F}^{\mathrm{T}}\left[\begin{array}{llll}
1 & 1 & 1 & 1 \\
1 & 1 & 1 & 1 \\
1 & 1 & 1 & 1 \\
1 & 1 & 1 & 1
\end{array}\right] \Phi .
$$

Note that the approximations for

$$
\left(\frac{\partial \phi}{\partial x}\right)_{0,0}
$$

and

$$
\left(\frac{\partial \phi}{\partial y}\right)_{0,0}
$$

are identical to the expressions (C-9) and (C-10) for the first finite differences with respect to $x$ and $y$.

Substituting (C-28), (C-29), (C-30) and (C-31) into (C-27) and performing the variation yields

$$
\begin{gathered}
\frac{1}{4 A_{i}-\frac{1}{2}, j-\frac{1}{2}}\left\{\left[\begin{array}{rrrr}
0 & -1 & 0 & 1 \\
1 & 0 & -1 & 0 \\
0 & 1 & 0 & -1 \\
-1 & 0 & 1 & 0
\end{array}\right]\left(Y^{T}+X X^{T}\right)\left[\begin{array}{rrrr}
0 & 1 & 0 & -1 \\
-1 & 0 & 1 & 0 \\
0 & -1 & 0 & 1 \\
1 & 0 & -1 & 0
\end{array}\right]\right\} \\
-\frac{A_{i-\frac{1}{2}, j-\frac{1}{2}}}{16}\left[\begin{array}{llll}
1 & 1 & 1 & 1 \\
1 & 1 & 1 & 1 \\
1 & 1 & 1 & 1
\end{array}\right] F
\end{gathered}
$$


Expanding and numbering the comers of the element as indicated yields:
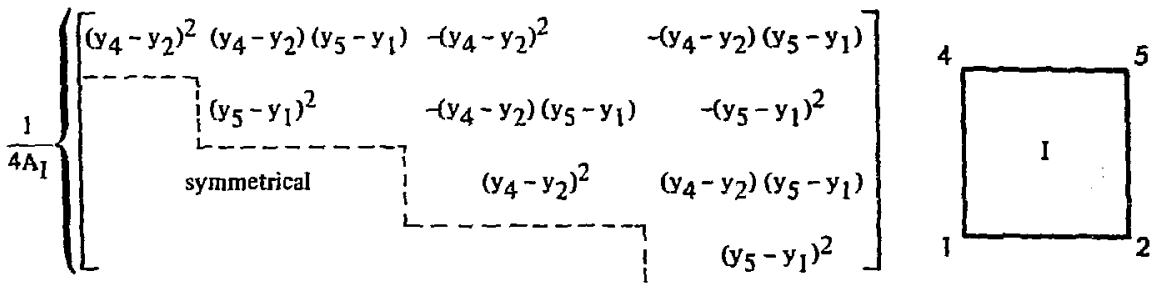

$+\left\{\begin{array}{ccc}\left(x_{4}-x_{2}\right)^{2}\left(x_{4}-x_{2}\right)\left(x_{5}-x_{1}\right) & -\left(x_{4}-x_{2}\right)^{2} & -\left(x_{4}-x_{2}\right)\left(x_{5}-x_{1}\right) \\ \hdashline\left(x_{5}-x_{1}\right)^{2} & -\left(x_{4}-x_{2}\right)\left(x_{5}-x_{1}\right) & -\left(x_{5}-x_{1}\right)^{2} \\ \text { symmetrical } & \left(x_{4}-x_{2}\right)^{2} & \left(x_{4}-x_{2}\right)\left(x_{5}-x_{1}\right)\end{array}\right\}\left\{\begin{array}{l}\phi_{1} \\ \phi_{4} \\ \phi_{5} \\ \\ \end{array}\right.$

$$
-\frac{A_{1}\left(f_{1}+f_{2}+f_{4}+f_{5}\right)}{16} \quad\left\{\begin{array}{l}
1 \\
1 \\
1 \\
1
\end{array}\right\} .
$$

Note the similarity between the $\phi_{5}$ equation for the $I^{\text {th }}$ tinite element and the $A_{1}$ contribution to the finite difference equation (C-17). For the grid used in the finite difference equations, it is apparent that the finite element equation for $\phi_{5}$ is

$$
\begin{aligned}
& -\sum_{i=1}^{9} \frac{\alpha_{i}}{4} \phi_{i}-\frac{1}{16}\left[A_{I} f_{1}+\left(A_{I}+A_{I I}\right) f_{2}+A_{I I} f_{3}+\left(A_{1}+A_{I I I}\right) f_{4}+\left(A_{I}+A_{I I}+A_{I I}+A_{I V}\right) f_{5}+\left(A_{I I}+A_{I V}\right) f_{6}\right. \\
& \left.\quad+A_{I I I} f_{7}+\left(A_{I I I}+A_{I V}\right) f_{8}+A_{I V} f_{9}\right]=0,
\end{aligned}
$$

where $\alpha_{i}$ is as defined in Eq. (C-17). If lumped forces had been used in the derivation of the finite element equation, i.e., concentrated forces, $F_{i j}$, given by

$$
F_{i j}=\frac{1}{2} A_{i j} f_{i j} \text {, }
$$

Eq. (C-34) multiplied by $-2 / A_{i, j}$ would have been identical to Eq. (C-17). Therefore, the lumped force isoparametric element and the finite difference equations from Ref. 1 yield identical difference equations at interior points.

\section{Boundary Conditions}

In the finite element calculations, for boundary grid points on $\mathrm{C}_{1}$ set

$$
\phi+\mathrm{h}=0 \text {. }
$$


For boundary grid points on $C_{2}$, the following equation holds if element $I$ has one (or more) sides on $C_{2}$ :

$$
\begin{aligned}
& \delta\left\{\iint \frac{1}{2}\left[-\left(\frac{\partial \phi}{\partial x}\right)^{2}-\left(\frac{\partial \phi}{\partial y}\right)^{2}+2 f \phi\right]|J| d s d t\right. \\
& -\frac{1}{2} \int_{-1}^{+1}(g \phi)_{t}=-1 \ell_{1,2} d s-\frac{1}{2} \int_{-1}^{+1}(g \phi)_{s}=+1 \ell_{2,5} d t \\
& \left.-\frac{1}{2} \int_{+1}^{-1}(g \phi)_{t}=+1 \ell_{5,4} d s-\frac{1}{2} \int_{+1}^{-1}(g \phi)_{s}=-1 \ell_{4,1} d t\right\}=0
\end{aligned}
$$
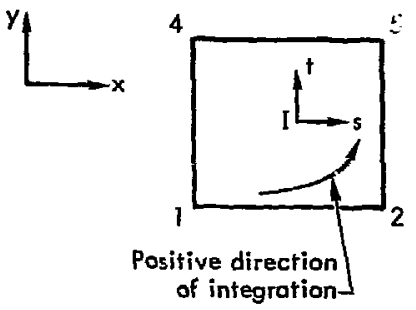

where

$$
\ell_{i, j}=\sqrt{\left(x_{i}-x_{j}\right)^{2}+\left(y_{i}-y_{j}\right)^{2}}
$$

Note that $\delta(g \phi)=0$ except on a $C_{2}$ boundary.

The area integral is evaluated as before to yield, after the variation is performed, a term as given by expression (C-33).

The line integrals are evaluated as follows:

$$
\text { let } g=H^{T} G, G=\left\{\begin{array}{l}
g_{1} \\
g_{4} \\
g_{5} \\
g_{2}
\end{array}\right\} \text {, }
$$

$g_{i}=0$ unless point $i$ is on $C_{2}$ (including end points).

Also,

$$
\phi=\mathrm{H}^{\mathrm{T}} \Phi, \Phi=\left\{\begin{array}{l}
\phi_{1} \\
\phi_{4} \\
\phi_{5} \\
\phi_{2}
\end{array}\right\}
$$


Then

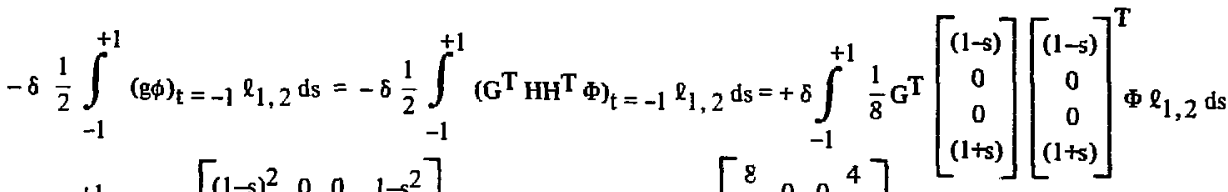

$$
\begin{aligned}
& =+6 \int_{-1}^{+1} \frac{1}{8} G^{T}\left[\begin{array}{cccc}
(1-s)^{2} & 0 & 0 & 1-s^{2} \\
0 & 0 & 0 & 0 \\
0 & 0 & 0 & 0 \\
1-s^{2} & 0 & 0 & (1+s)^{2}
\end{array}\right] \Phi \ell_{1,2} d s=+\delta \frac{1}{8} G^{T}\left[\begin{array}{cccc}
\frac{8}{3} & 0 & 0 & \frac{4}{3} \\
0 & 0 & 0 & 0 \\
0 & 0 & 0 & 0 \\
4 & 0 & 0 & \frac{8}{3}
\end{array}\right] \Phi Q_{1,2} \\
& =Q_{1,2}\left[\begin{array}{c}
\left(\frac{1}{3} g_{1}+\frac{1}{6} g_{2}\right) \\
0 \\
0 \\
\left(\frac{1}{3} g_{2}+\frac{1}{6} g_{1}\right)
\end{array}\right]
\end{aligned}
$$

Similarly,

$$
\begin{aligned}
& -\delta \frac{1}{2} \int_{-1}^{+1}(g \phi)_{\mathrm{s}=+1} l_{2,5} \mathrm{dt}=-l_{2,5}\left[\left(\begin{array}{c}
0 \\
0 \\
\left(\frac{1}{3} g_{5}+\frac{1}{6} g_{2}\right) \\
\left(\frac{1}{3} g_{2}+\frac{1}{6} g_{5}\right)
\end{array}\right]\right. \text {, } \\
& -\delta \frac{1}{2} \int_{1}^{-1}(g \phi)_{\mathrm{t}=+1} \ell_{5,4} d s=-l_{5,4}\left[\begin{array}{c}
0 \\
\left(\frac{1}{3} g_{4}+\frac{1}{6} g_{5}\right) \\
\left(\frac{1}{3} g_{5}+\frac{1}{6} g_{4}\right) \\
0
\end{array}\right], \\
& -\delta \frac{1}{2} \int_{1}^{-1}(g \phi)_{s=-! \ell_{4,1} d t=+\ell_{4,1}}\left[\begin{array}{c}
\left(\frac{1}{3} g_{1}+\frac{1}{6} g_{4}\right) \\
\left(\frac{1}{3} g_{4}+\frac{1}{6} g_{1}\right) \\
0 \\
0
\end{array}\right] .
\end{aligned}
$$

(C-40)

(C-41)

$-47-$ 
The contribution of element $I$ to the boundary conditions at $I$ and 2 are given by rows $I$ and 4 of expression (C-33) and expressions (C-38) through (C-41) respectively. At point 2 of the figure at the right, for example. adding the contributions of elements $I$ and II yields

$$
\begin{aligned}
& \frac{1}{4 A_{1}}\left\{\left[\left(y_{4}-y_{2}\right)\left(y_{5}-y_{1}\right)+\left(x_{4}-x_{2}\right)\left(x_{5}-x_{1}\right)\right]\left(\phi_{1}-\phi_{5}\right)\right. \\
& \left.+\left[\left(y_{5}-y_{1}\right)^{2}+\left(x_{5}-x_{1}\right)^{2}\right]\left(\phi_{4}-\phi_{2}\right)\right\} \\
& -\frac{1}{4 A_{I I}}\left\{\left[\left(y_{5}-y_{3}\right)^{2}+\left(x_{5}-x_{3}\right)^{2}\right]\left(\phi_{6}-\phi_{2}\right)\right. \\
& \left.+\left[\left(y_{5}-y_{3}\right)\left(y_{6}-y_{2}\right)+\left(x_{5}-x_{3}\right)\left(x_{6}-x_{2}\right)\right]\left(\phi_{5}-\phi_{2}\right)\right\}-\frac{A_{I}\left(f_{1}+f_{2}+f_{4}+f_{5}\right)}{16}+\frac{A_{I I}\left(f_{2}+f_{3}+f_{5}+f_{6}\right)}{16} \\
& +l_{1,2}\left(\frac{1}{6} g_{1}+\frac{1}{3} g_{2}\right)+l_{2,3}\left(\frac{1}{3} g_{2}+\frac{1}{6} g_{3}\right)=0
\end{aligned}
$$

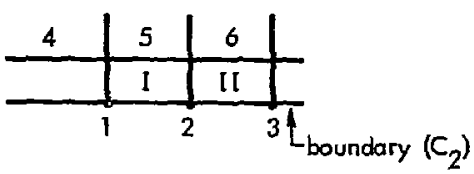

Boundary conditions of the above form can also be used with the finite difference equations. Equation (C-42), reformulated with "lumped" values of $g$ on the boundary, is equivalent to writing backward difference equations with respect to outward normal at the boundary. It should be noted that the boundary conditions written in the above form will result in a symmetric inatrix.

\section{GENERAL COMMENTS}

It has been shown that the finite difference method of Ref. 1 and the isoparametric element (with lumped forces) and one-point integration lead to the same difference equations when applied to Poisson's equation.

It follows, since the expressions for the desivatives are identical, that the two methods will give identical difference equations when applied to the 2-D plane stress or plane strain elasticity equations. The boundary conditions derived for the finite element provided a means of writing finite difference equations that result in a symmetric matrix.

As discussed in Ref: $l$, an "hour glass" distortion pattern in the rectangular grid can sometimes fail to produce the strain components in the elasticity problem. This difficulty is therefore also present in the iscparametric element. However, if the strain energy in the isoparametric element is integrated with the $2 \times 2$ Gaussian metnod, as is normally done, the "hour glass" distortion pattern causes no difficulty. This difficulty is also eliminated in the tinite difference method by evaluating the contour integrals for the first partial derivatives over the four triangles formed by three sides of the quadrilateral and averaging as discussed in Ref. 6 . The analogoiss problem in three dimensions is treated in the previous section where tetrahedrons are used to form a tensor viscosity.

Since the two methods yield the same equations (not including the adjustments required to eliminate the "bour glass" pattern) the choice between the two methods is one of convenience. 


\section{Appendix D \\ HEAT 3D, a Code for Calcularing Therrnal Diffusion \\ in Three Dimensions}

\section{THERMAL STRESS ANALYSIS}

In solving 3-D thermoelastic problems, we have assumed the following principles of Boley and Weiner: ${ }^{7}$

- The temperature diffusion at any time can be determined independently of the intermediate displacements; i.e., material thermal properties are not a function of displacements;

- Displacements are small, so that products of displacement gradients can be neglected;

- The materiais are elastic.

Under these assumptions a given temperature distribution causes a stress tensor represented by:

$$
\begin{aligned}
& \sigma_{\mathrm{xx}}=\lambda \Delta V+2 \mu \epsilon_{\mathrm{xx}}\left(\lambda+\frac{2}{3} \mu\right) 3 \alpha \Delta \phi \\
& \sigma_{\mathrm{yy}}=\lambda \Delta V+2 \mu \epsilon_{\mathrm{yy}}\left(\lambda+\frac{2}{3} \mu\right) 3 \alpha \Delta \phi \\
& \sigma_{\mathrm{zz}}=\lambda \Delta \mathrm{V}+2 \mu \epsilon_{\mathrm{zz}}-\left(\lambda+\frac{2}{3} \mu\right) 3 \alpha \Delta \phi \\
& \tau_{\mathrm{xx}}=\mu \epsilon_{\mathrm{xy}} \\
& \tau_{\mathrm{yz}}=\mu \epsilon_{\mathrm{yz}} \\
& \tau_{\mathrm{zx}}=\mu \epsilon_{\mathrm{zx}} \\
& \Delta \mathrm{V}=\epsilon_{\mathrm{xx}}+\epsilon_{\mathrm{yy}}+\epsilon_{\mathrm{zz}} \\
& \left(\lambda+\frac{2}{3}\right)=\mathrm{K}=\text { bulk modulus }
\end{aligned}
$$

where $\lambda$ and $\mu$ are the Lamie constants, $\epsilon_{x, y, z}$ are the strain components, $\alpha$ is the coefficient of thermal expansion, $\Delta \phi$ is the temperature change, and $\Delta \nabla$ is the dilatation. In practice, we want to find the maximal stress created $b$ larticular time-dependent loading. Boley and Weiner's first assumotion allows us to solve therr: elastic prc $o_{1}$ ems numerically in two distinct steps. Since temperature is not a function of subsequent displai $i^{2}$ ment, we firsi solve the temperature diffusion problem by using HEAT 3D. The code segisters temperatures at various times; the time showing the greatest temperature change causes the greatest thermal stress. The second step, determining the value of this stress, is to run the stress analysis code, SAP, inserting the temperatures found in the first step. 
Suppose we are given an object, $R$, with an initial temperature, $T_{0}$, prescribed on a portion of its surface. $S$. We know that the iemperature at any point on the object at any time after we begin observation satisfies the equation:

$$
-\rho c \frac{\partial \phi}{\partial t}+k \nabla^{2} \phi+q=0
$$

$$
\phi(x, y, x, 0)=T_{0}
$$

In $R$ and on $S$,

$$
k \frac{\partial \phi}{\partial n}=h_{c}\left(\phi-T_{\infty}\right)
$$

where

$$
\begin{aligned}
& \rho=\text { density of the material } \\
& c=\text { the heat capacity } \\
& k=\text { the thermal conductivity } \\
& q=\text { the heat source } \\
& h_{c}=\text { the coefficient of thermal convection } \\
& T_{\infty}=\text { the ambient temperature. }
\end{aligned}
$$

It can be shown that finding the $\phi$ that satisfies (D-1), (D-2), and (D-3) is equivalent to finding a $\phi$ that minimizes the functional

$$
\frac{1}{2} \int_{R} k\left[\left(\frac{\partial \phi}{\partial x}\right)^{2}+\left(\frac{\partial \phi}{\partial y}\right)^{2}+\left(\frac{\partial \phi}{\partial z}\right)^{2}\right] d \mu-\int_{R} \rho c \frac{\partial \phi}{\partial t} \phi d \mu+\int_{R} q \phi d \mu+\int_{S} h_{c}\left(T_{\infty}-\frac{1}{2} \phi\right) d \nu
$$

over a class of sufficiently smooth functions. We will find our temperature distribution function, $\phi$, by using this minimization principle.

Our first step in the procedure is to partition the object into small hexahedrons, $R_{k}$. We will denote the temperature at any time at the eight vertices of $R_{k}$ by $\phi_{1}$ through $\phi_{8}$. Then we can approximate $\phi$ at any point in $\mathbf{R}_{\mathbf{k}}$ by

$$
\phi(x, y, z, t)=\sum_{j=1}^{8} h_{j}(x, y, z) \phi_{j}(t)
$$

where $h_{j}(x, y, z)$ is an appropriately chosen interpolating function. 
In the HEAT 3D code we have chosen a multilinear interpolating scheme given by

$$
\begin{aligned}
& h_{1}(s, t, u)=\frac{1}{8}(1+s)(1+t)(1+u) \\
& h_{2}(s, t, u)=\frac{1}{8}(1-s)(1+t)(1+u) \\
& h_{3}(s, t, u)=\frac{1}{8}(1-s)(1-t)(1+u) \\
& h_{4}(s, t, u)=\frac{1}{8}(1+s)(1-t)(1+u) \\
& h_{5}(s, t, u)=\frac{1}{8}(1+s)(1+t)(1-u) \\
& h_{6}(s, t, u)=\frac{1}{8}(1-s)(1+t)(1-u) \\
& h_{7}(s, t, u)=\frac{1}{8}(1-s)(1-t)(1-u) \\
& h_{8}(s, t, u)=\frac{1}{8}(1+s)(1-t)(1-u)
\end{aligned}
$$

where $(s, t, u)$ are points on a cube of side-length 2 , centered at the origin. This interpolating function can be viewed as a mapping from $(s, t, u)$-space to $(x, y, z)$-space, as illustrated in Fig. D-1 .

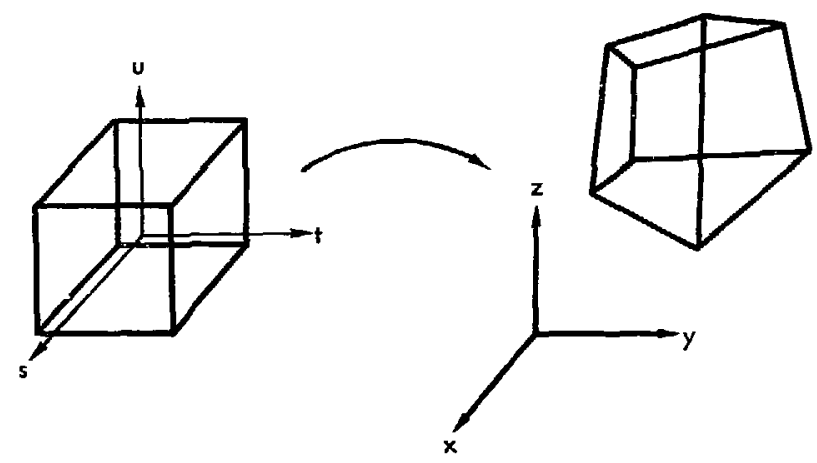

Fig. D-1. The eight-node block in $(x, y, z)$-space and in $(s, t, n)$-space.

For notational convenience

$$
H=\left(h_{1}, h_{2}, \ldots, h_{8}\right)
$$

We proceed with our minimization scheme by substituting the nght-hand-side of (D-5) for $\phi$ on each $\mathbf{R}_{\mathbf{k}}$ in $\mathbf{R}$. (Note that in terms of the above notation (D-5) becomes $\phi=H \Phi$, where $\Phi^{T}=\left(\varphi_{1}, \varphi_{2}, \ldots, \varphi_{8}\right)$.) Using the properties of the derivative we find

$$
\frac{\partial \varphi}{\partial x}=\sum_{i=1}^{8} \frac{\partial h_{i}}{\partial x} \varphi_{i}
$$


and

$$
\left(\frac{\partial \varphi}{\partial x}\right)^{2}=\sum_{i=1}^{8} \sum_{j=1}^{8}=\frac{\partial h_{i}}{\partial x} \varphi_{i} \frac{\partial h_{j}}{\partial x} \varphi_{j}=\Phi^{T}\left[\frac{\partial h_{i}}{\partial x} \frac{\partial h_{j}}{\partial x}\right]_{\Phi}=\Phi^{T} \frac{\partial H^{\top}}{\partial x} \frac{\partial H}{\partial x} \Phi .
$$

The first integral in (D-4) becomes

$$
\frac{1}{2} \int_{-1}^{1} \int_{-1}^{l} \int_{-1}^{1} k\left\{\Phi\left(\left[\frac{\partial H^{T}}{\partial x} \frac{\partial H}{\partial x}\right]+\left[\frac{\partial H^{T}}{\partial y}-\frac{\partial H}{\partial y}\right]+\left[\frac{\partial H^{T}}{\partial z} \frac{\partial H}{\partial z}\right]\right) \Phi\right\}|J| d s d t d u
$$

where $J$ is the usual Jacobian, i.e.,

$$
|J|=\operatorname{det}\left[\begin{array}{lll}
\frac{\partial x}{\partial s} & \frac{\partial y}{\partial s} & \frac{\partial z}{\partial s} \\
\frac{\partial x}{\partial t} & \frac{\partial y}{\partial t} & \frac{\partial z}{\partial t} \\
\frac{\partial x}{\partial u} & \frac{\partial y}{\partial u} & \frac{\partial z}{\partial u}
\end{array}\right]
$$

Since $\Phi$ is a function of time only, (D-9) becomes

$$
\frac{1}{2} k \Phi^{T} \int_{-1}^{1} \int_{-1}^{1} \int_{-1}^{1}\left(\left[\frac{\partial H^{T}}{\partial x} \frac{\partial H}{\partial x}\right]+\left[\frac{\partial H^{T}}{\partial y} \frac{\partial H}{\partial y}\right]+\left[\frac{\partial H^{T}}{\partial z} \frac{\partial H}{\partial z}\right]\right)|J| d s d t \text { du } \Phi .
$$

From elementary calculus and the definition of $H$,

$$
\left[\begin{array}{ll}
\frac{\partial}{\partial x} & H \\
\frac{\partial}{\partial y} & H \\
\frac{\partial}{\partial z} & H
\end{array}\right]=J^{-1}\left[\begin{array}{l}
\frac{\partial H}{\partial s} \\
\frac{\partial H}{\partial t} \\
\frac{\partial H}{\partial u}
\end{array}\right]
$$

Hence, we finally have (D-10) in a form we can calculate, denoted by

$$
\frac{1}{2} \Phi^{T} \mathbf{k \Phi}
$$

For each $\mathbf{R}_{k}$ we apply this same approximating technique to each integral in (D-4). The second integral becomes

$$
\int_{-1}^{1} \int_{-1}^{1} \int_{-1}^{1} \rho c \dot{\phi}^{\mathrm{T}} \mathrm{H}^{\mathrm{T}} \mathrm{H} \phi|\mathrm{J}| \mathrm{ds} d t d u
$$


or, after doing the integration.

$$
\dot{\phi}^{T} C \Phi
$$

By $\dot{\phi} \mathbf{T}$, we mean the vector:

$$
\left[\frac{d}{d t} \varphi_{1} \cdot \frac{d}{d t} \varphi_{2} \ldots \ldots \frac{d}{d t} \varphi_{8}\right] .
$$

For the third integral, which gives the heat loading, we have

$$
\int_{-1}^{1} \int_{-1}^{1} \int_{-1}^{1} Q H \Phi|J| d s d t d u
$$

or simply

$\overline{\mathbf{Q}} \boldsymbol{\Phi}$.

The fourth integrol inputs the convection boundary data and, since it is a surface integral, it must be handled in a slightly different manner. Let

$$
\hat{\phi}=\left[\begin{array}{l}
\hat{\phi}_{1} \\
\hat{\phi}_{2} \\
\hat{\phi}_{3} \\
\hat{\phi}_{4}
\end{array}\right] .
$$

where $\hat{\phi}_{j}$ are the temperatures on the surface of $R_{k}$ where convection is taking place.

Let

$$
\hat{H}=\left\{h_{1}(s, t,-1), h_{2}(s, t,-1), h_{3}(s, t,-1), h_{4}(s, t,-1)\right]
$$

In terms of this notation, the fourth integral becomes

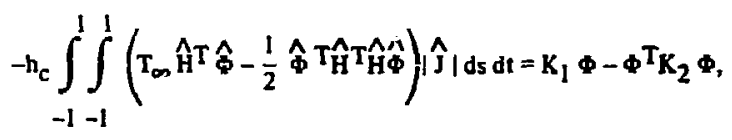

where $|\hat{\mathrm{J}}|$ is the appropriate Jacobian.

After the above procedure, and after taking the direct sum over all the $\mathbf{R}_{\mathbf{k}}$, we obtain a matrix expression

$$
\Phi T_{K \Phi}+\dot{\phi} T_{C \Phi}-\bar{Q} \Phi-\mathbf{K}_{\mathbf{i}} \Phi-\Phi^{T} \mathbf{K}_{2} \Phi
$$


that approximates (D-4). With this discretized form of (D-4) we can find a minimizing $\Phi$ that approximates $\phi$, the desired answer. From classical calculus the minimum of (D-15) occurs at its critical points or on the boundary functions. The critical points of the system satisfy

$$
\mathbf{K} \Phi+C \dot{\Phi}-\overline{\mathbf{Q}}-\mathbf{K}_{1}-\mathbf{K}_{2} \Phi=0
$$

To see whether the critical points or the boundary points are ininjmizing. we check the convexity of the equation. Differentiating again gives

$$
\mathbf{K}-\mathbf{K}_{\mathbf{2}} \text {, }
$$

which is positive as long as the conductivity exceeds the convection condition, i.e. whenever the body has a heat input. This condition is satisfied for all physical applications in which we are interested.

Thus we are left with solving for the critical points. i.e., we want to solve the system of ordinary differential equations

$$
K \Phi+C \dot{\phi}-\bar{Q}-K_{1}-\check{K}_{2} \phi=0
$$

with initial conditions $\Phi=T_{0}$.

This system can be solved in numerous ways, but the technique we will wse is the simple forward difference scheme

$$
\frac{C}{\Delta t}\left(\Phi^{n+1}-\Phi^{n}\right)=\vec{Q}+K_{1}-\frac{1}{2}\left(K_{2}+K\right)\left(\Phi^{n+1}+\Phi^{n}\right)
$$

After substituting

$$
\psi=\frac{1}{2}\left(\varphi^{n+1}-\Phi^{n}\right)
$$

we obtain the symmetric matrix eq̣ı ations

$$
\left(\mathrm{K}_{2}+\mathrm{K}+\frac{2 \mathrm{C}}{\Delta \mathrm{t}}\right) \Psi=-\overline{\mathrm{Q}}+\frac{2 \mathrm{C}}{\Delta \mathrm{t}} \Phi^{\mathrm{n}} \cdot \Phi^{\mathrm{n}+\mathrm{l}}=2 \boldsymbol{\Psi}-\boldsymbol{\Phi}^{\mathrm{n}} .
$$

This method is implicit; convergence to a solution of (D-1), (D-2), and (D-3) is guaranteed.

\section{CODE ORGANIZATION}

The computer progran for solving the heat diffusion equation has been broken into two logically distinct parts. The first part generates the coefficient mitrices in $\mathrm{Eq}$. (D-16). To accomplish this the code reads in the coordinates of the discretized object. the bourdary and initial conditions, the material properties, and the information that describes each hexahedron. The coordinates, boundary, and initial conditions are read from a file, LNK, in a binary form in a format standardized for the stress analysis codes. Graphicaily it has the indicated form, where $n$ is the number of node points. 
The condition code tells the program whether the temperatures are to be tted as a source load. a fixed boundary condition, or a convection temperat! ' he code for a particular node is 0 . the temperature is a source; if 1 , the tem. is a boundary condition and held constant; and if 2 , it is considered to bi a surface-convection ambient temperature. This binary file can be generated using the VMESH $3 D$ code and the BOUNDER code described previously unde- SAP.

The element description and the material properties are read from an ASCII File, TPI, which can be generated by the code ORGANIZER (see section on SAP). An element description from this code is compatible with that of the SAP stress analysis code. This first part of the code does the appropriate integration over individual elements and the direct sum on the elemental matrices, as required. The code writes as output an ASCII file, TP2, which contains the matrices, $C, \overline{\mathrm{Q}}$, $T_{0}$, and $T_{\infty}$, and half of the matrix, $K$. (Only half is needed by symmetr' 1 At

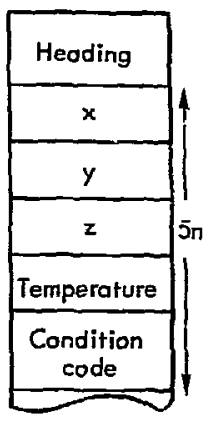
this point, any special modifications to the TP2 file can be made using a.i: siandard ASCII file test editor.

The second half of the code reads the information on TP2, along with the time-step and the number of timesteps, and assembles it into the form of Eq. (D-21). The code uses the banded positive-definite symmetric matrix solver, DISBAND, to invert the left-hand side of (D-21). The code then performs the time-step operation, printing out the answer for each time step. The answer file can be edited for input to SAP using the code, FEVER. (See section on SAP.)

\section{CODE LIMITATIONS}

Limitations of the code fall into two types, a tandwidth size limit and a tine-step limitation. The bandwidth size limit of 560 is imposed by the core management scheme of the matrix inversion subroutine. The code automatically changes core boundaries for optimal performance by the subroutine, but a bandwidth in excess of 560 will cause the code to print an error message and quit. The code will handle matrices of arbitrary cardinality.

filthough stability is not a problem with the simple differencing scheme we have chosen, the accuracy of the solution appears to put rather stringent conditions on the choice of $\Delta t$. In test problems we have run, discontinuities in initial conditions give rise to bad overshoot in the approximation. In many problems the $\Delta t$ could be varied with time during the problem solution; the choice of fixed $\Delta t$ is costly, creating a practical limit on the size of problems that may be nun accurately. This limitation can be overcome by using one of the many sophisticated ordinary differential equation integrators now available.

\section{TEST PROBLEMS}

In order to verify the accuracy of the code HEAT $3 D$ we have run two test problems for which the theoretical solutions are known.

\section{Linear Thermal Conduction}

We studied the thermal conduction in a square beam with heating on one end and a heat sink on the other. The mathematical formulation of the problem is 


$$
\frac{\partial \phi}{\partial t}=\nabla^{2} \phi
$$

Boundary conditions:

$$
\begin{aligned}
& \phi(0, y, z, t)=1 \\
& \phi(1, y, z, t)=0 .
\end{aligned}
$$

As may be seen in Fig. D-2, $\partial \phi / \partial \mathrm{n}=0$ for all other surfaces.

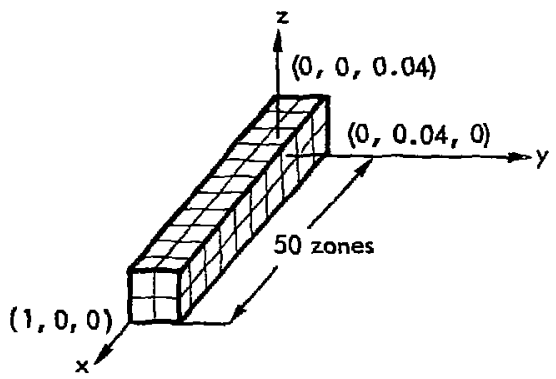

Fig. D-2. Geometry for calculating one-dimensional heat flow using the three-dimensional code HEAT 3D.

This problem has a Fourier series solution of

$$
\phi(x, t)=(1-x)-\frac{2}{\pi} \sum_{n=1}^{\infty} \frac{\sin (n \pi x)}{n} e^{-n^{2} \pi^{2} t}
$$

When run on the HEAT 3D code with $\Delta x=\Delta y=\Delta z=0.02$ and $\Delta t=0.0002$, we obtained the results presented in Table D-1.

\section{Two Dimensional Heat Flow}

A test of the code accuracy for solving a problem in two space dimensions and time can be obtained by imposing the first Fourier harmonic to the general solution of the heat equation on a unit square, as an initial condition. The theoretical result for this injtial condition is an exponential decay in temperature as time progresses. Expressed in mathematical symbols, we used the code to solve

$$
\frac{\partial \phi}{\partial t}=\nabla^{2} \phi
$$

with initial conditions

$$
\phi(x, y, 0)=\sin \pi x \sin \pi y
$$


Table D-1. Comparison of the theoretical solution and the code solution for a one dimensional heat flow problem.

(a) Temperatures at time $=0.01$

\begin{tabular}{ccc}
\hline $\mathrm{X}$ & Theoretical & HEAT 3D \\
\hline .0 & 1.000 & 1.000 \\
.1 & .479 & .480 \\
.2 & .155 & .158 \\
.3 & .034 & .035 \\
.4 & .008 & .005 \\
.5 & .000 & .000 \\
.6 & .000 & .000 \\
.7 & .000 & .000 \\
.8 & .000 & .000 \\
.9 & .000 & .000 \\
1.0 & .000 & .000 \\
\hline
\end{tabular}

(b) Temperatures at time $=0.1$

\begin{tabular}{ccc}
\hline $\mathrm{X}$ & Theoretical & HEAT 3D \\
\hline .0 & 1.000 & 1.000 \\
.1 & .823 & .829 \\
.2 & .655 & .663 \\
.3 & .502 & .511 \\
.4 & .371 & .379 \\
.5 & .263 & .269 \\
.6 & .178 & .183 \\
.7 & .114 & .117 \\
.8 & .066 & .068 \\
.9 & .030 & .031 \\
1.0 & .000 & .000 \\
\hline
\end{tabular}

and boundery conditions

$\phi(1, y, t)=\phi(0, y, t)=\phi(x, 1, t)=\phi(x, D, t)=0$

A 20 by 20 grid is used.

The theoretical solution to the problem is

$\phi(x, y, t)=\sin \pi x \sin \pi y \mathrm{e}^{-2 \pi^{2} t}$.

Graphically, the initial conditions ase shown in Fig. (D-3a). Figures D-3b and D-3c show the temperature distribution at later times.

Table D-2 gives the corresponderice between the code results and the then.etical solution along a diagonal of the square. 

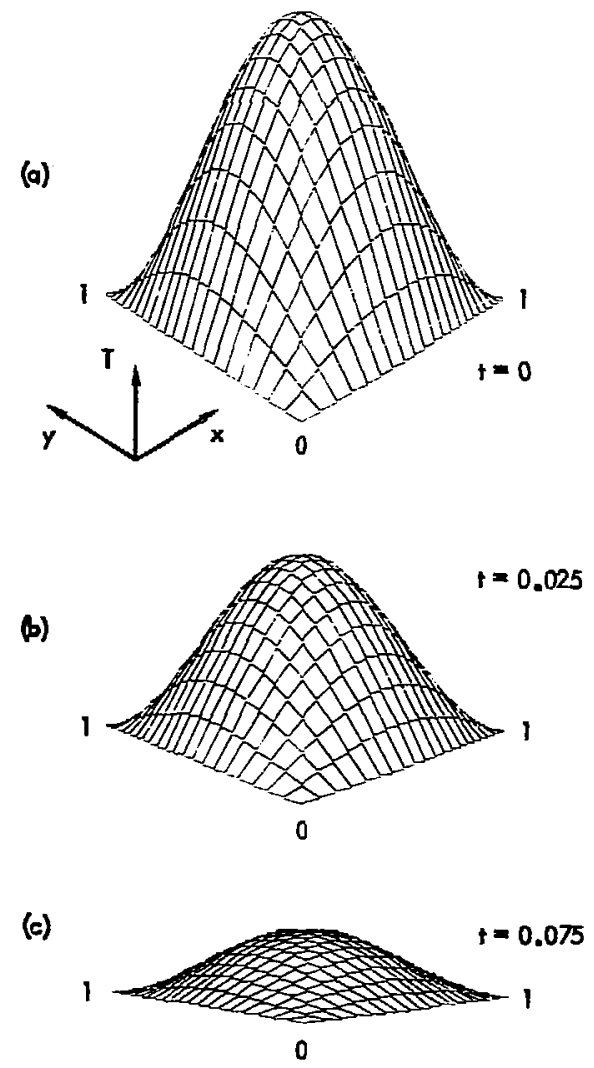

Fig. D-3. Temperature as a function of $x, y$ for different times. 
Table D-2. Comparison of the theoreticat and the code solutions for a two dimensional heat flow problem.

\begin{tabular}{|c|c|c|c|c|c|}
\hline \multicolumn{2}{|c|}{ Position } & \multicolumn{2}{|c|}{$t=0.02$} & \multicolumn{2}{|c|}{$\mathrm{t}=0.1$} \\
\hline $\mathbf{X}$ & $\mathbf{Y}$ & HEAT 3D & Theoretical & HEAT 3D & Theoretical \\
\hline .0 & .0 & .000 & .000 & .000 & .000 \\
\hline .05 & .05 & .017 & .016 & .003 & .003 \\
\hline .10 & .10 & .064 & .064 & .013 & .013 \\
\hline .15 & .15 & .139 & .139 & .029 & .029 \\
\hline .20 & .20 & .233 & .233 & .048 & .048 \\
\hline .25 & .25 & .337 & .337 & .070 & .069 \\
\hline .30 & .30 & .442 & .441 & .092 & .091 \\
\hline .35 & .35 & .536 & .535 & .111 & .110 \\
\hline .40 & .40 & .610 & .609 & .127 & .126 \\
\hline .45 & .45 & .658 & .657 & .137 & .136 \\
\hline .50 & .50 & .675 & .674 & .140 & .139 \\
\hline .55 & .55 & .658 & .657 & .137 & .136 \\
\hline .60 & .60 & .610 & .609 & .127 & .126 \\
\hline .65 & .65 & .536 & .535 & .111 & .110 \\
\hline .70 & .70 & .442 & .441 & .092 & .091 \\
\hline .75 & .75 & .337 & .337 & .070 & .069 \\
\hline .80 & .80 & .233 & .233 & .048 & .048 \\
\hline .85 & .85 & .139 & .139 & .038 & .037 \\
\hline .90 & 90 & .064 & .064 & .013 & .013 \\
\hline .95 & .95 & .016 & .016 & .003 & .003 \\
\hline 1.00 & 1.00 & .000 & .000 & .000 & .000 \\
\hline
\end{tabular}




\author{
Appendix E \\ VMESH 3D - A Three-Dimensional Zoner Program for \\ Semiauromatic Generation of Three Dimensional Meshes
}

\title{
INTRODUCTION
}

In running computer simulations of physical phenomena it is nesessary to describe the object under analysis to the computational program. Frequently this input data consists mostly of a finite number of geometric crordinates that constitute a "discrete-mesh" approximation of the object. Preparation of such meshes or zonings is a tedious and error-prone process, and niay be so costly as to inhibit tine running of problems. The following describes a semi-automatic zoning program, VMESH 3D, that greatly simplifies the task of generating 3D meshes.

VMESH 3D is an implementation of some of the ideas contained in MESH 3D, ${ }^{8}$ a progrem written for Bettis Corporation by Hutula and Zeiler. As it now stands, VMESH $3 D$ is less versatile than MESH 3D. This is primarily due to the zoning limitations imposed by the finite difference code, wherein a plane of the mesh taken in any of the three logical directions $(i, j, k)$ will have the same number of node points as any other plane taken in the same direction. However, as in MESH 3D, the data generated to describe the mesh consist of the three $\langle x, y, z)$ geometric coordinates asseciated with each of the eight nodes of each zone. VMESH 3D does not concern itself with elements, assuming this generation is done in the finite element code itself.

\section{PROGRAM DESCRIPTION}

If you want a zoning program to which you can say something like, "Zone up a semi-ellipsoid with a $1 / 4$ in. hole, $17^{\circ}$ off the principal axis with...", then VMESH 3D is not for you. VMESH 3D requires the user to "decompose" his problem into subobjects, or blocks, that are simple to detine. Then, under his guidance, it joins blocks together to construct inore and more complex objects. This procedure is probably the most direct way to construct such complex meshes with a minimum of time and effort, and the objects describable in such a way are alnost unlimited.

The program basically performs two functions: definition and construction. In the aefinition phase the program accepts a user-supplied geometric description of a block and autematically subdivides it into the userspeciffed number of zones. This definition process may be thought of as analogous to deformirig a rectangular rubber brick to fit the geometry of the subobject. ${ }^{8}$ The construction phase consists of joining the subobjects or blocks along compatible surfaces to form the final mesh.

VMESH 3D, like MESH 3D, uses two coordinate systems, integer and real. The real space is the normal right-handed Cartesian coordinate system $(x, y, z)$. The integer coordinate system $(j, k, i)$ is, however. not the same as in MESH 3D but is relative to each particular block only (i.e., each node of a block has a j, $k$, i coordinate, with $j$ varying fastest. Node points go from $j=1$ through JPT in a line, from $k=1$ through KPT for each different line in a plane, and from $j=1$ through IPT for each plane). In the illustration, for example. JPT $=\mathrm{KPT}=\mathrm{IPT}=4$. 


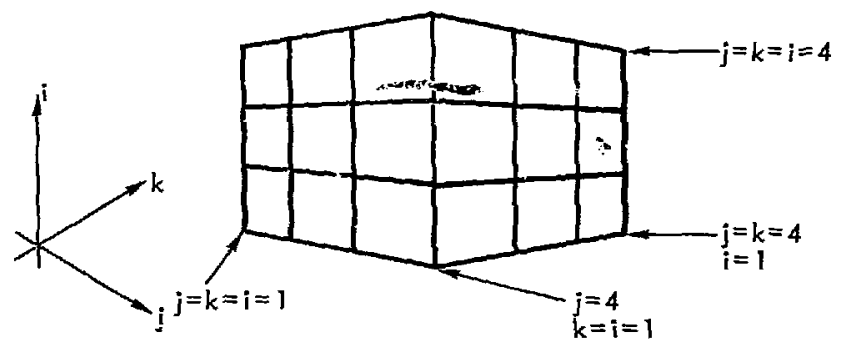

- The integer coordinates used in the tri-quadratic linear interpolation help determine the geometric coordinates of the node points. The reader should note at this point that this integer space is more or less independent of geometric space ( $x$ is not always $j$, etc.), and that the object can be defined such that it varies fastest in any direc$\operatorname{tion}_{1} x, y$, or $z$.

\section{DETAILS OF PROGRAM COMMANDS}

Various commands enable the user to convey his wishes to the program. Taken together, they allow him to construct the complex meshes required. In general, the user creates a block by using the DEFINE command and supplying both the geometric coordinates of certain key points on the block and the number of points in each direction. (Those familiar with MESH 3D will recognize this as their DEFINE and MAP combined.) Using the UPDATE command allows the user to change the geometric coordinates of individual points. (ERASE is not implemented.) Two individual blocks can then be joined by using one of the three commands, COMBINE, MERGE, or UNITE. There are alse provisions in VMESH 3D for two as yet unimplemented operations, COPY to define blocks by rotating existing blocks and JOIN to do complex assembling.

\section{DEFINE}

The DEFINE command is the only way to create a block. The input required to define a block is as follows:

Line No.

Input

\section{DEFINE}

$$
\text { Block number, JPT, KPT, IPT }
$$

Key point number (1-27), $x, y, z$

NOTE: For all input lines, there is no column orientation.

All symbols and numbers must be blank delimited. All

$x, y, z$ data will be read as floating point numbers; e.g., 6

will be read as 6.0. The keypoints may be input in any

order, but only 27 such lines will be read. (See Fig. E-1.) 
The block is assigned the particular block number. and is divided iniu thie spyctified number of nodes in each direction. (Note that the number of zones in each direction is one less than the number of nodes.) The block thus defined will hrive a total of JPT *KPT*IPT nodes, numbered from 1 to $\mathrm{JPT}^{*} \mathrm{KPT} \mathrm{T}^{*} \mathrm{IPT}$, and will consist of IPT planes of size JPT*KPT. The nodes are numbered by proceeding first in the $j$-direction, second in the $k$-direction, and last in the i-direction.

The first two input lines define the block in integer space to the program. The program then uses the geometric coordinates of the $27 \mathrm{key}$-points to map this block into real space. The key-points are located cin the block as shown in Fig. E-2, and all 27 must be input. The resulting mesh appears in Fig. E-3.

The mapping from integer to real ssace is basically the tri-quadratic interpolation scheme used in MESH 3D with 7 extra key-points being used in the VMESH 3D scheme. The edges of the block are fit to parabolic curves defined by the three edge points, and the six surface points enable the user to make the sides convex or concave.

It is recommended that one read the MESH 3D report $^{8}$ for a complete description of the mapping. Two important points should be kept in mind. First, the points between the block corners are not necessarily midpoints. Those that are midpoints produce even zoning along that edge. Those that are closer to one corner than the other compress the zoning along that edge toward the near corner and expand it toward the far corner. The closer the off-center point is to one corner, the finer will be the zones in that region and the more coarse will be the zones in the opposite comer.

Figure E-3 shows two very similar blocks illustrating this principle. The upper block was produced by the input in Fig. E-1. Representative nodes are numbered. Both blocks have JPT $=5, \mathrm{KPT}=7$, and IPT $=9$. However, on the lower block we set the $z$ coordinates for key points 17 through 24 (and 26) equal to 1.5 (not 2), causing the

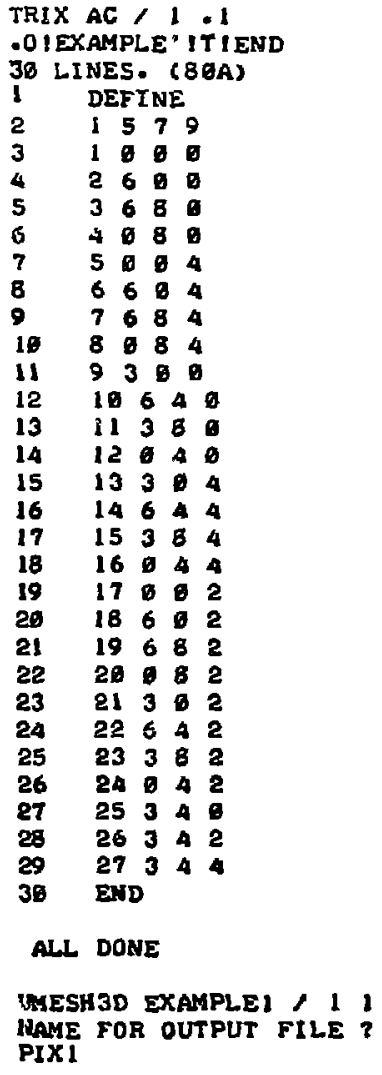

\section{ALL DONE}

Fig. E-1. A sample DEFINE command in VMESH 3D. The key points could have been specified in any order, but we chose to do them in sequence for safety 3 sake. planes to settle slightly toward the bottom.

Second, the program does a parabolic approximation to fit the three points along each edge. In order to avoid a serious mismatch between the approximation and a desired curve it is important to subdivide the problem enough to minimize the fitting error.

Figure E-4 illustrates the importance of breaking the problem into enough parts. The top curve was produced by doing a single DEFINE, forming a 3-point parabolic approximation to a semicircular arc. The second curve, a much better approximation, is the result of doing two DEFINEs and then a MERGE (see below). The number of node points is the same for both figures. 


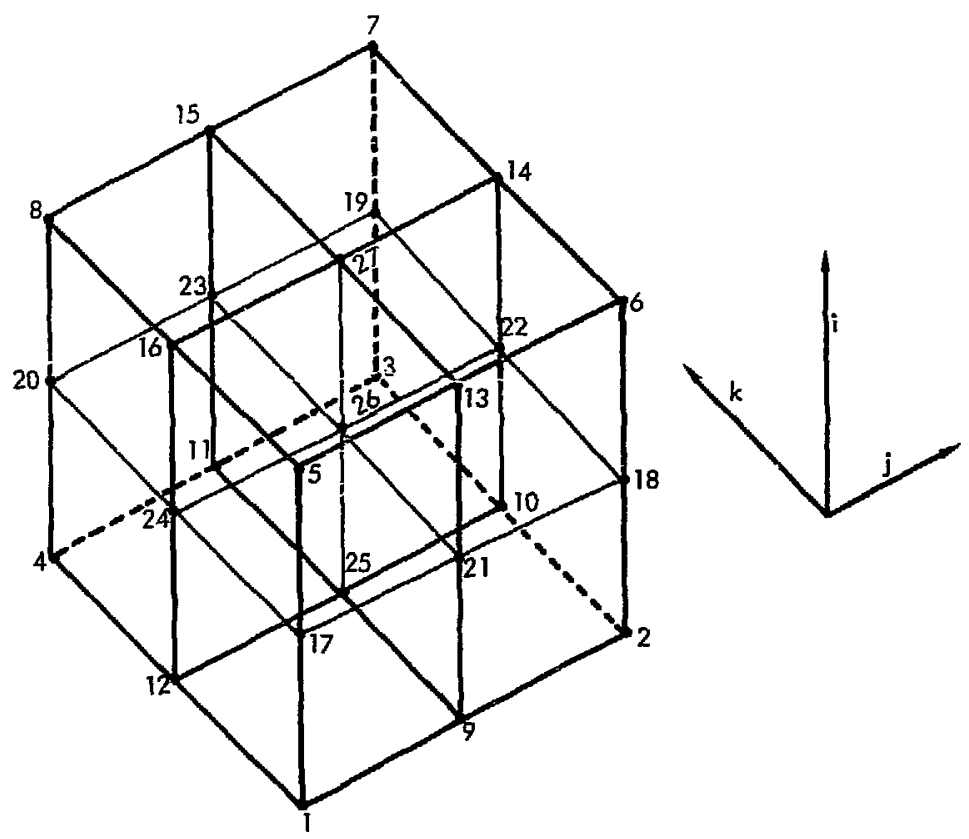

Fig. E-2. Locations of block key-points for VMESH 3D.

\section{UPDATE}

Once a block is defined, the geometric coordinates of individual nodes in the block can be altered by using the update command. The user must calculate the number of node(s) he wants to change, but this is a fairly simple task. The input required to do an update is:

\section{UPDATE}

Block-Number Numbet-of-Points-to-be-Changed(n)

$(3$ thru $n+2)$

Node-Number XYZ

Note that all three coordinates must be specified in order to change a point, even if only one is actually being altered. Figure E-3(b) could have been formed by using UPDATE on the block illustrated in Fig. E-3(a).

\section{MERGE}

The MERGE comnand actually combines two simple blocks into one. The blocks must have similar plane sizes (in node count) in the direction they are being joined. For example, if the user wishes to join block $A$ to block $B$ in the J-direction, then IPTA*KPTA must equal IPTB*KPTB. The nodes on the common face of the second block are eliminated; the rest of the points are merged into the first block's fle, and the second file is destroyed. If one defines two separate blocks in such a way that they have a plane in common (exactly the same number of points with the same coordinates) then the MERGE command returns a single block with the duplicate points eliminated. To do a MERGE, VMESH 3D needs: 


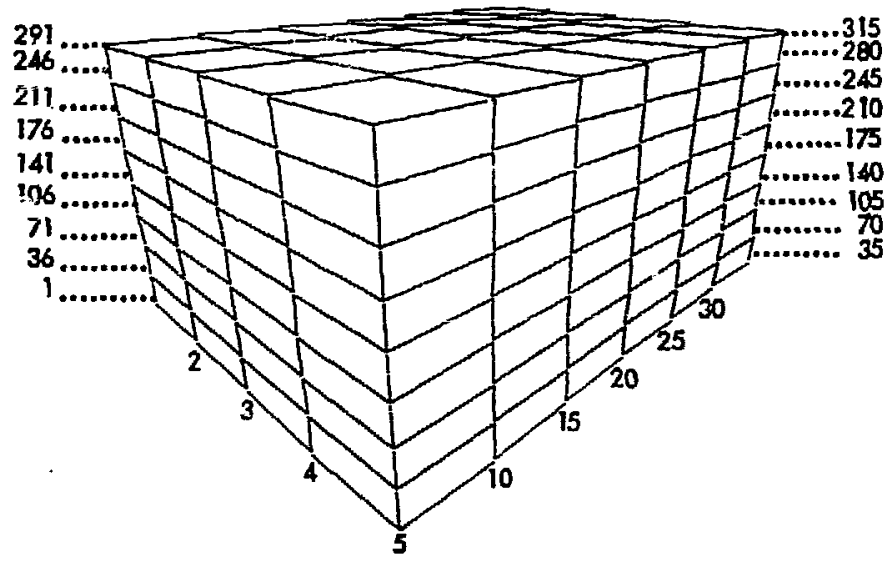

(a)

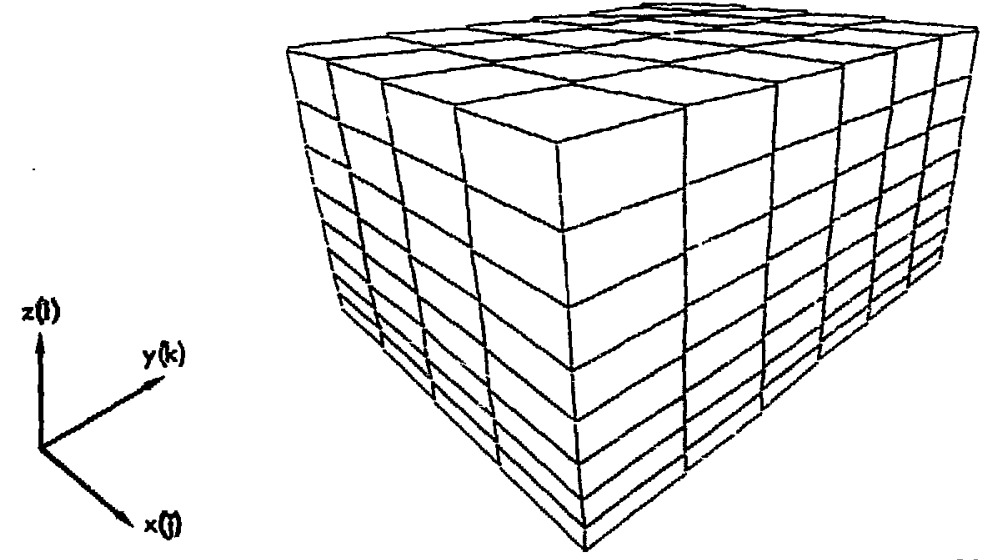

b)

Fig. E-3. Rectangular meshes produced by: (a) DEFINE illustrated in Fig. E-1. (b) UPDATE command operating on the original DEFINE, changing $z$ for points $17-24,26$ from 2.0 to 1.5 .

\section{(1) MERGE \\ (2) Block-Number-A Block-Number-B-Direction (i, j, or k)}

One caveat is in order here. The user should keep in mind how he definted the blocks in both geometric and integer space before performing a merge. The second point (the first being eliminated) of the second block in the joining direction is always joined to the lost point of the first block in that same direction. If one mixes blocks $A$ and $B$ on input, or gives an incorrect direction number, strange 'end-around' effects may occur or plane sizes might not match. 


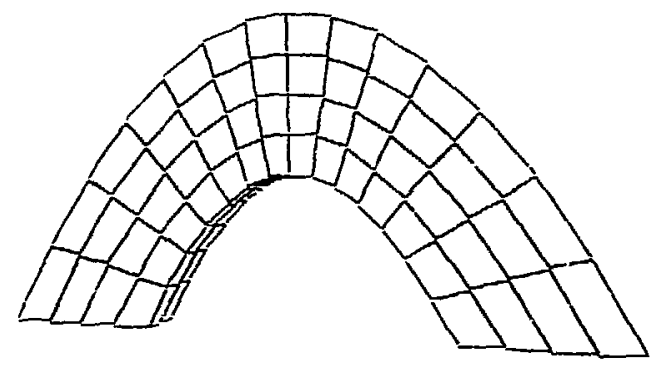

6)

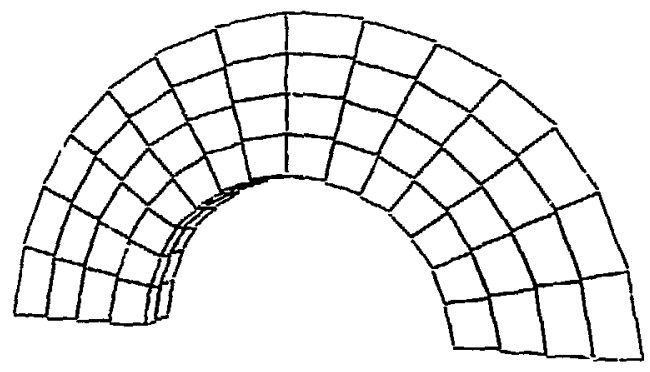

\$)

Fig. E-4. Parabolic approximations to a semicircular arc. (a) A single DEFINE. (b) Two $90^{\circ}$ DEFINEs, MERGEd.

Figures E-5 to E-1 I illustrate the process of building up complicated objects out of relatively simple blocks. In Fig. E-5 a relatively complex ooject is defined fairly easily with VMESH 3D. We used four DEFINEs, one for each quarter of the hemisphere, and three MERGEs. Note that we had only two blocks defined at once, e.g. DEFINE 1, DEFINE 2. MERGE 1 and 2, DEFINE 2, MERGE 1 and 2, DEFINE 2, MERGE 1 and 2.

Figures E-6 to E-1 1 go step by step through a more intricate assembly, using commands DEFINE, MERGE, and COMBINE (See below.) The finished mesh, representing a songitudinal half section of a flanged $90^{\circ}$ pipe elbow, consists of three separate blocks in one file. The planning in this example is simple and direct, but inefficient. A more efficient plan would be to define one flange, add the pipe, and then add the other flange.

\section{COMBINE}

When a block is created, there is a header on the block data file indicating the number of node points in the block, the number of points per plane, IPT, JPT, KPT, etc. There is also an indicator of how many blocks the file contains. The COMBINE command allows the user to add a block to the data file without altering the block being added or the blocks already in the file, and increment the block number in the header. The data needed for the COMBINE is:

(1) COMBINE

(2) Block-number-A Block-number-B 

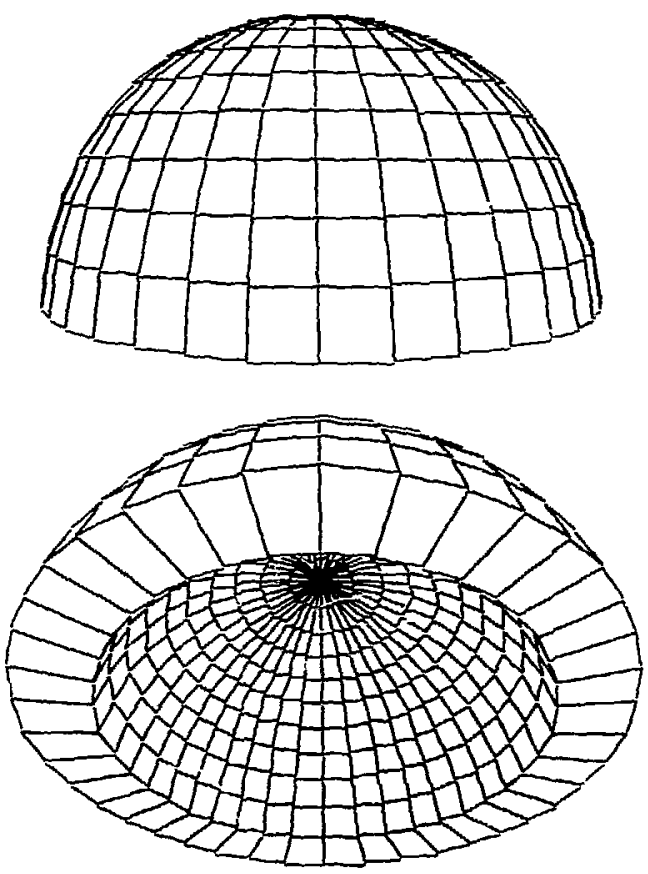

Fig. E-S. A hemispherical dome mesh produced with four DEFINEs and three MERGEs.

This instructs the program to write the block-B data into the block-A file, update the header, and destroy the block B-fle. The following diagram should help to illustrote what's going on here:
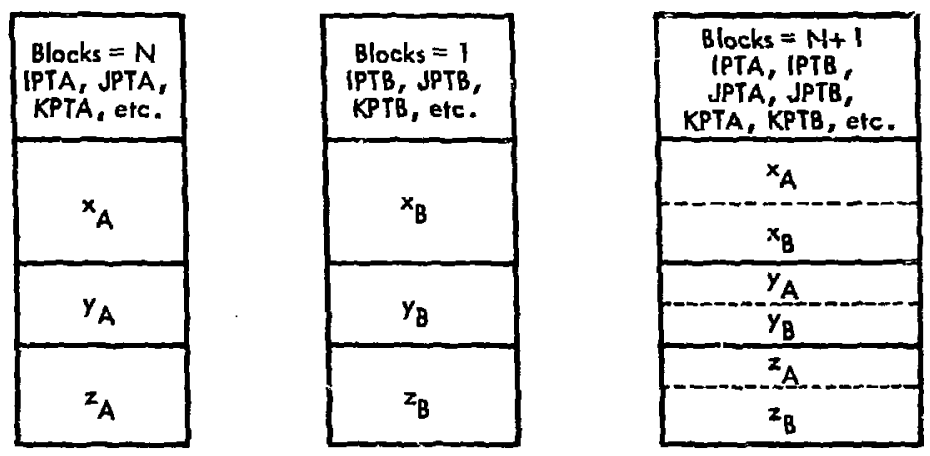

Note that this is the only way to combine two blocks along faces with dissimilar plane sizes, and that the points will be duplicated along such interfaces. A simple algorithm will elininate these duplicate poinis for finiteelement genetation. 


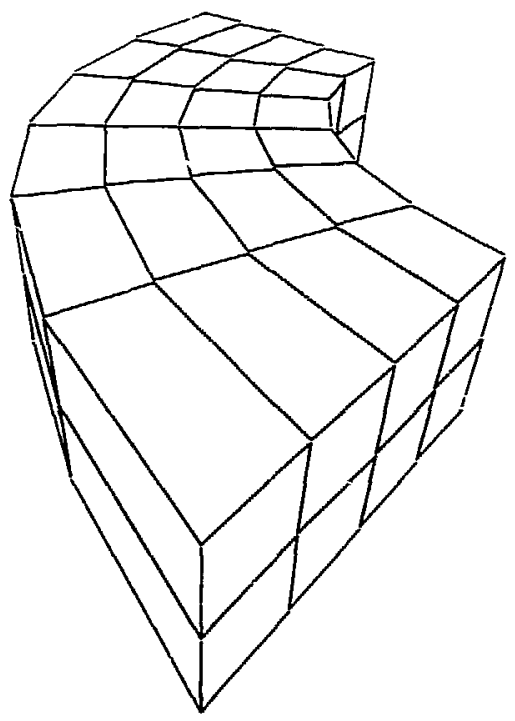

Fig. E-6. The first step in defining a flanged pipe. DEFINE 1, a $90^{\circ}$ annular segment.

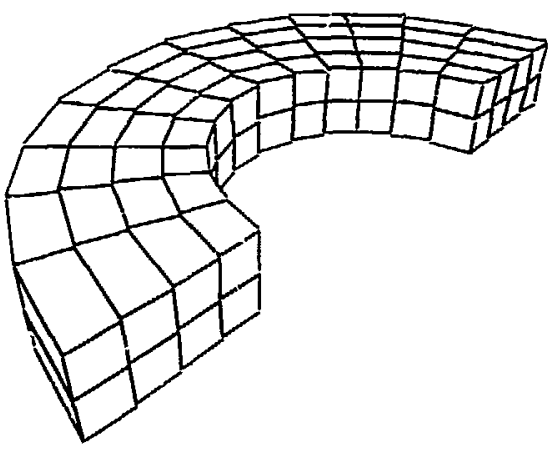

Fig. E-7. DEFINE 2 and MERGE 1 and 2. This produces a single logical block, representing a semicircular arnular segment. 

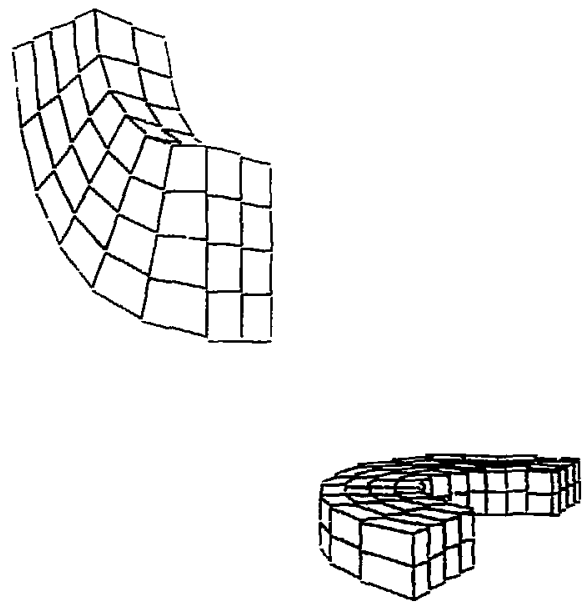

Fig. E-8. DEFINE 2, adding a separate $90^{\circ}$ annular segment.
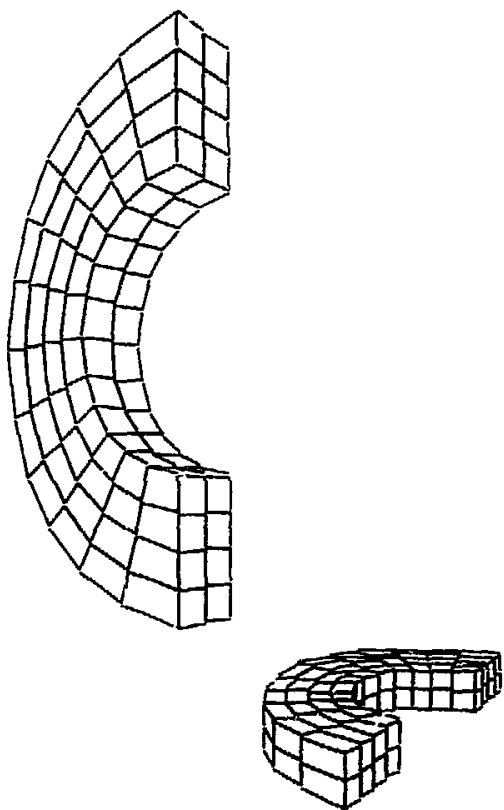

Fig. E-9. DEFINE 3, and MERGE 2 and 3, producing two semicircular annular segments. These two fugical blocks ran't be merged, but we can COMBINE I and 2. This gives two blocks in a single file. 
The UNITE command has virtually the same effect as the MERGE command, except the plane of the second block is not eliminated. Instead, the two blocks are soined by inserting a single layer of zones between the commo it planes. The input to UNITE is essentially the same as for MERGE:

\section{(1) UNITE \\ (2) Block-Numbet-A Block-Number-B Birection}

\section{Output File}

The output file consists of a 512-ward header and then all the x's for all blocks, all the $y$ 's for all blocks, and then all the $z$ 's. The file is absolute binary with no formatting; i.e., it uses no FORTRAN I/O. The header contains the number of blocks ( 19 max.) and the total number of node points in the entire object. It also contains, for each block, the total number of node points, the number of points per $j-k$ plane, IPT, JPT, and KPT.

\section{RUNNING THI: PROGRAM}

VMESH 3D exists as a running program for the LLL 7600. It can be run with input being supplied from TTY, or from a card file of data commands. The progran can also switch between the two modes by using the commands TTY and CARDS, as appropriate. To fire up the program, type:

\section{VMESH3D $/ \mathrm{t} v$ or VMESH3D filename $/ \mathrm{t} v$}

In the first case, the progrom will ask if you wish to operate from TTY or a card file, initially. In the second case, you are specifying a card file and the program goes there to get its first command.

The first command is always DEFINE, and it produces a block file (usually numbered 1). A second DEFINE will produce another block file. As new blocks are defined they may be entered into the original file by commands COMBINE, MERGE, or UNITE. No more than ejght separate block files can be defined at any one time, but with proper planning you should rarely need more than two or three. The key thing to remember here is to plan everything out before you use ine program.

At the end all the blocks must be in a single file. This file may contain as many as ten separate blocks, i.e. up to nine COMBINE commands. MERGE and UNITE commands, since they lump blocks together, do not affect this total. 


\section{Appendix F \\ VPIX 3D - A 3-D Graphic Program for Interactive \\ Display of Three Dimensional Objects}

\section{INTRODUCTION}

Simulating physical phenomena by finite difference (or finite element) computer programs places many burdens on the user. Foremost among these are verification of input data and interpretation of output results. In $3 \mathrm{~V}$ programs the problems become acute, since it is difficuit to convey $3 D$ information on conventional 2D media, especially as numbers. (If a 2D dunp is an array, with rows being one dimension and columns the other, where do we put the third dimension?) Hence, graphical display is a prime necessity in running and understanding 3D codes. VPDX 3D provides the user with many of the required capabilities in the graphics area.

\section{GENERAL PROGRAM DESCRIPTION}

VPIX 3D generates pictures of 3D objects in proper perspective with all hidden lines removed. The program is interactively capable of rotating, translating, and scaling the object in the field of view. VPLX 3D sends its pictures to a variety of vatput devices, including teletypewriter (TTY), dd80, television monitor display system (TMDS), dd80A, and indirectly, grey-level TMDS. It displays 3D meshes, either as line drawings or half-tone pictures shaded from a light source, and can be used to display input mesh to check their validity, or the coordinates of node points output by the main program to display distortions caused by displacement. The program also generates pictures with shading done according to some problem variable (e.g., temperature) and pictures with surface contours determined, as in the shading, by an arbitrary variable.

The subroutines that perform the perspective calculation, hidden line removal, and packing of data required by the various output media were written by Mike Archuleta of the LLL Computer Graphics Group. ${ }^{9}, 10$ See also Ref. 11. In particular, the subroutines that remove hidden lines (no mean task) are an implementation of the Watkins' algorithm ${ }^{12}$ for the LLL 7600 network (OCTOPUS). Their rapid pe:formance makes feasible the semi-interactivity of the program.

\section{DATA FILE}

The program operates from TTY, with the user supplying commands that indicate how he wishes to look at the object, and where he wants the picture to go. It expects as input a data file (on disk) with the following characteristics in order: a 512 word header, all $x$ coordinates, all $y$ coordinates, all $z$ coordinates, and finally all variable values. (Note that the variable values need not be present in the file if the user simply wishos to display the mesh geometry.) In graphical form, the file lonks like this:

\begin{tabular}{|c|}
\hline Header \\
\hline$x$ \\
\hline$y$ \\
\hline$z$ \\
\hline $\begin{array}{c}\text { Variable } \\
(e . g . \text { temp.) }\end{array}$ \\
\hline
\end{tabular}

*Not yet on-line at press time. 
The header contains the following data:

\begin{tabular}{cl} 
WORD (abs. disk addr.) & \multicolumn{1}{c}{ Information } \\
\hline 0 & $\begin{array}{l}\text { Number of "blocks" in the file. } \\
1\end{array}$ \\
$2-11$ & Tolal number of node points for all blocks together. \\
$12-21$ & Number of node points per plane for each block. \\
$22-31$ & Number of planes per block (IPT) \\
$32-41$ & Number of joints in j-direction for each block (JPT). \\
$42-51$ & Number of points in k-direction for each block (KPT). \\
$52-512$ & Available for future use.
\end{tabular}

(Note that there are a maximum of ten blocks in a problem and that the $x-y-z$ data starts at absolute disk address $1000_{8}$ ).

An explanation is in order. VPIX 3D was written primarily as a service routine for the finite difference code, HEMP 3D, and the finite element code, SAP. The meshes for these problems are very regular in the sense that they are composed of eight-node "bricks" arranged in a regular fashion or "block." A block is typified as consisting of a number of planes (taken in one of the three logical directions, $i, j$, or $k$ ), with the number of node points being corstant in each plane. An object may consist of several blocks. Figure F-l presents 1 simple twoblock problem and the header associated with it.

One might question what this type of organization buys the user. To see any advantage, one must first look at the hidden-line subroutines. These routines operate on polygons; clipping, sorting, storing them, etc. In this application, the polygons are simply the rectangular faces of the zones. Now, since our blocks are very regular, the algorithm used to pick the coordinates of these polygons (zones) out of the data file is relatively simple. What's more, we can easily pick out and pass to the hidden-line subroutines only those polygons on the surface. This simplifies their work and implies the possibility of displaying larger objects (i.e., the interior zones of the mesh are already known to be hidden; the fact needn't be discovered by the hidden-line algorithm).

\section{COORDINATE SYSTEM}

The data in the file is defined in a standard three-dimensional "right-handed" Cartesian coordinate system. Since the hidden surface algorithm perversely uses a left-handed system, the program will transform the data to present the picture correctly. In manipulating the picture, however, the user must emplov the left-handed system. Essentially, the eye is at the origin looking "down" the positive z-axis (into the screen) with positive y being up and positive $x$ to the right. The field of vision is limited to a pyramid defined $t y$ the planes, $z= \pm x$, and $z= \pm y$, Fig. F-2.

The initial view is a picture of the bottom of the object. It is up to the user to alter this view, if he so desires.

\section{CHANGING THE VIEW}

Usually no single picture reveals everything one wishes to know about a particular object. Therefore the program enables the ur $\cdots$; j rotate the object around its center point, move the object up or down, left or right, or toward or away from him, and to scale the object (zoom in or out). There is also a "slicing" option, allowing him to look at the interior of the object. The commands are input from the teletype and may be "stacked" (i.e., more than one command per TTY line). 

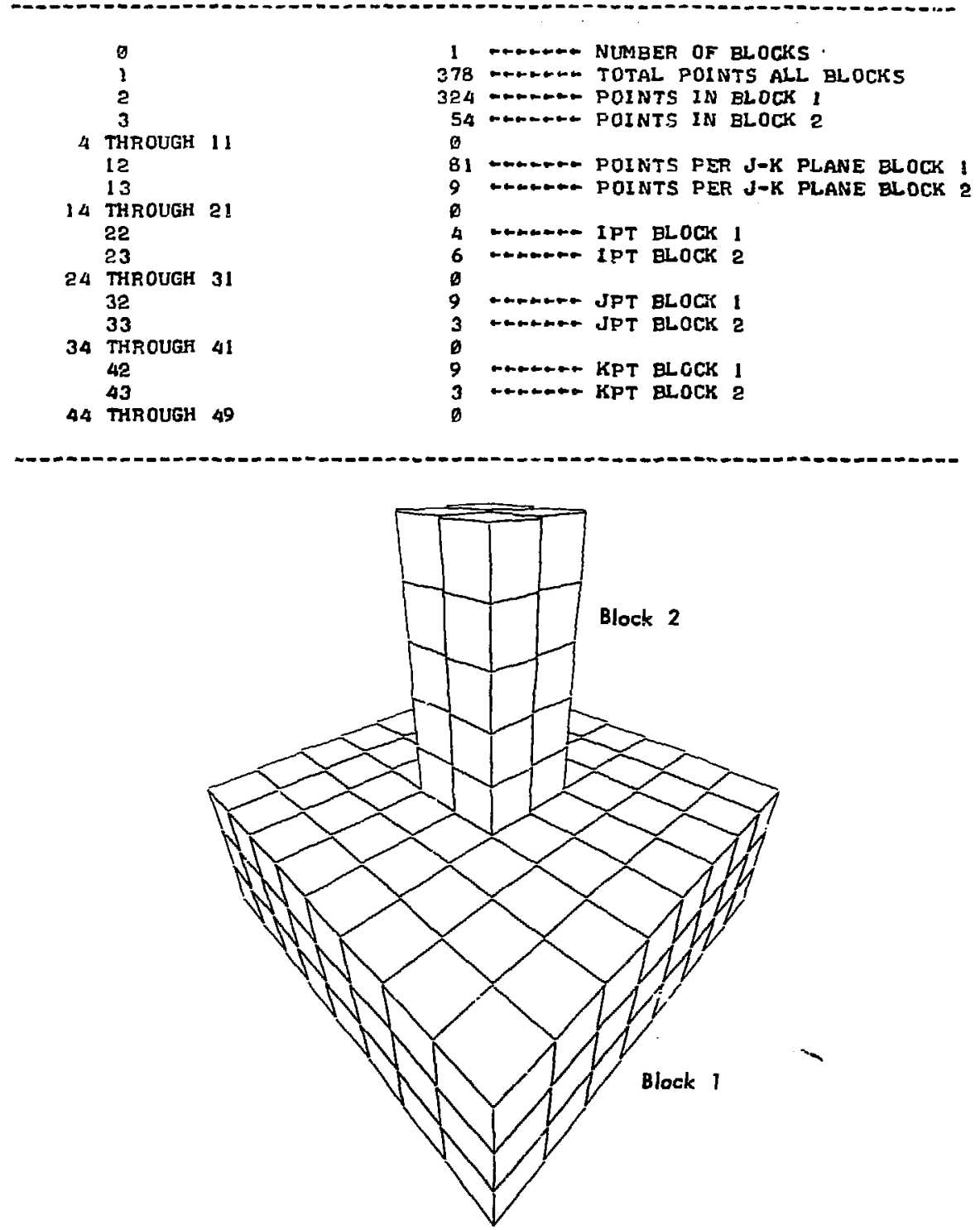

Fig. F-1. A simple two-block mesh and the VPLX 3D header associated with it. 


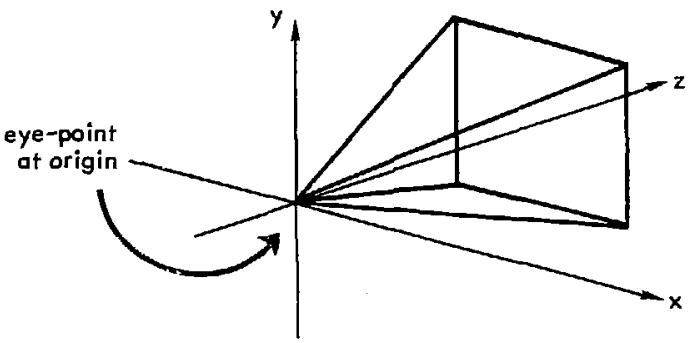

Fig. F-2. Cone of vision in VPIX 3D.

\section{Rotation Commands}

Figure F-3 depicts the results of commands that enable the user to revolve the object about its center point. The center point is located on the z-axis of the viewing system. It is sometimes helpful to imagine a separate "object coordinate system" associated with this point. The rotations can then be thought of as changing this system in relation to the coordinate system of the viewer. The user should practice with these commands until he is familiar with them, and must remember that they are not commutative. (A YAW 45, then PITCH 45, is not always the same view as a PITCH 45, then YAW 45.)

\section{Command Action}

$\mathbf{P} \pm \mathrm{n}$ or $\quad$ Rotate the object around its $\mathrm{x}$ axis by $\mathrm{n}$ degrees. If $\mathrm{n}$ is positive, the rotation PITCH $\pm \mathbf{n} \quad$ is in the direction shown (i.e., the top comes forward, the bottom recedes). A negative $n$ will move it in the opposite direction.

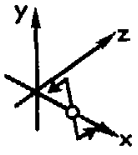

$\mathrm{Y} \pm \mathrm{n}$ or $\quad$ Rotate the object around its $\mathrm{y}$ axis by $\mathrm{n}$ degrees. As with all commands, a $\mathrm{YAW} \pm \mathrm{n} \quad$ positive $\mathrm{n}$ need not be preceded by the plus sign. A positive $\mathrm{n}$ will bring the night side forward and cause the left side to recede.

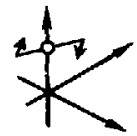

$R \pm n$ or ROLL $\pm n$ Rotate the object around its z-axis. A positive $n$ will move the top of the object to the right and down, and the bottom to the left and up.

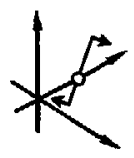

\section{Translation Commands}

The translation commands are very simple to understand. They move the object along axes. Where the rotation commands enable one to see all sides of an object, the translation commands will move the object around in the field of view. 


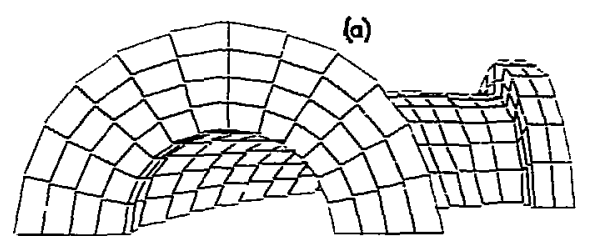

(b)

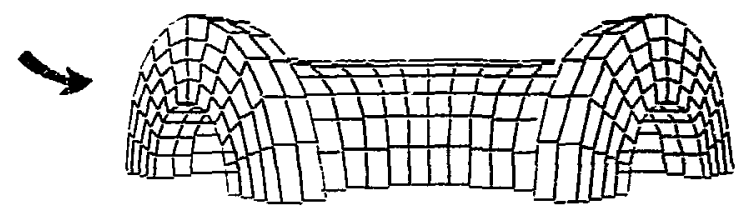

(c)
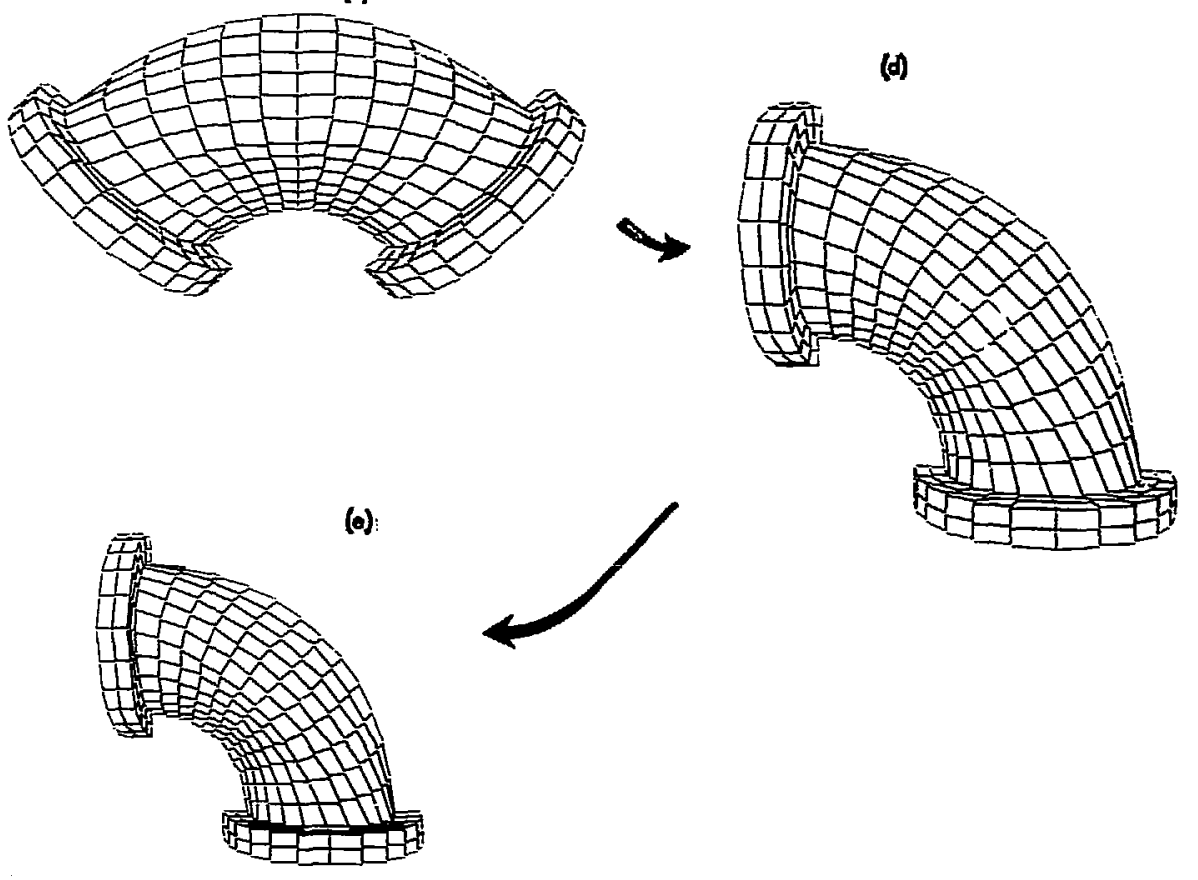

Fig. F-3. Successive views obtained with various VPIX 3D commands. (a) Original position. (b) YAW 45 or Y 45. (c) PITCH 90 or P 90. (d) ROLL 45 or R 45. (e) SCALE .75 or S .75. 
TX $\pm n$ Move the objeat right or left $n$ units. A positive $n$ moves it to the right: is negative $n$ to the left.

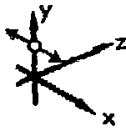

TY \pm n Move the object up or down n units. A positive a here moves it up; a negative $n$ moves it down.

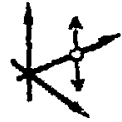

$\mathbf{T Z} \pm \mathbf{n}$ Move the object backward or forward $\mathrm{n}$ units. A positive $\mathrm{n}$ moves it into the screen; a nigalive n moves it out (similar to scaling).

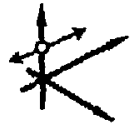

\section{Scaling Command}

There is a single command that holds the object in place and nakes it larger or smaller. This enables the user to concentrate on details of greatest interest to him.

Command

$\mathbf{s}$ or

SCALE n

\section{Action}

Multiplies the coordinates of the picture by $n$ while holding the center point fixed. Note: the command will accept a negative $n$ but this has the same effect as YAW 180 SCALE n.)

\section{The Slice Option}

The first part of the hidden linte algorithm removes all lines that lie outside the viewing "window" or screen, a process known as "clipping." Ordinarily, the routine clips to the planes establishing the viewing pyramid (i.e., $z= \pm x, \pm y)$. However, it can also clip to two other $z$-planes $(z=Z M A X, Z M I N)$ and thus remove even more of the picture. If the limits of the object being viewed are beyond the two planes in either, or both, directions (i.e., OBJMAX $>Z M A X$, OBSMIN $<Z M I N$ ), then part of the object is removed. Thus, one can "slice" through an object and examine its interior. Figure F-4 depicts a cube being sliced progressively along a . diagonal.

Howevs, this introduces a problem; i.e., the interior must be present before it can be examined. If you will remember, the program usually passes only the exterior coordinate; to the hidden-line subroutines, since it "knows" all interior points are hidden anyway. Now, since the user can slice the object arbitrarily, the program no longer knows which interior points are "automatically" hidden. Therelore it chooses the coward's way out; it passes the coordinates of all the polygons to the hidden line algorithm, and asks it to decide which are hidden. 

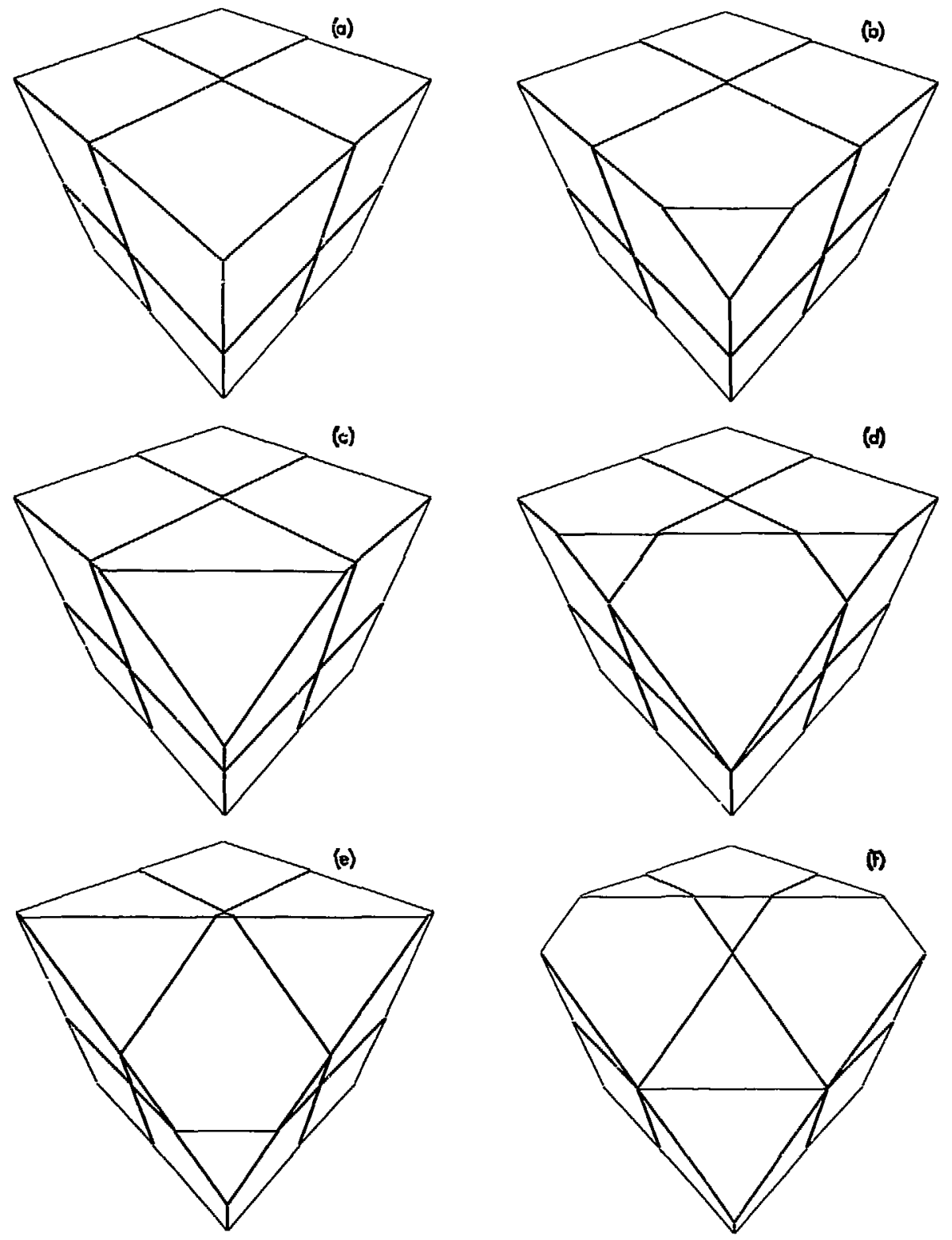

Fig. F-4. A cube progressively sliced along a body diagonal. 
The user should be well aware of the extra burden he is placing on the routine in doing a slice. There is a limit to the number of polygons the hidden-line subroutines can handle and, if the user chooses too large a slice. they will run out of space and send an error messige. The option is then to choose a smaller slice, or quit.

\section{Command}

User: SLICE

Routine: $\mathrm{ZMAX}=\mathrm{ZMIN}=$ TYPE NEW ZMAX ZMIN

User: $\mathbf{n} \mathbf{n}$

NOSL

\section{Action}

The routine will go into "slicing mode" and inform the user of the present ZMAX and ZMIN. It will then ask the user for a new ZMAX and ZMIN. The user then specifies the limits of the slice. and the routine will go about its business.

Tuin off the slicing option. Should be tumed off, whenever it is unneeded, for effieient operation.

\section{Contour Plotting Option}

In analyzing the output of 3D problems. it is often desirable to examine the value of certain problem variables at various locations on, or in the problem mesh. One method of doing this is to display lines of constant value, contours, on the problem grid. Figures 3 and 4 are examples of such plots. The contour plotting commands in VPLX 3D allow the user to specify the number of contour levels he wishes to calculate and to select among any of those levels for any single picture.

Command

CONTR n

HILO nhi nlo

\section{Action}

Turn on the contour option and set up n contour levels. This sets up an array, CLEVEL, with $n$ entries $(n<36)$. Where CLEVEL $(1)=$ MMN $+(((V M A X-V M I N))$ $(n-1) *([-1))$ CONLO $=$ VMIN.

The program will plot contours for all values of CLEVEL between CONHI and CONLO.

Thus. one can selectively delete or add contours by the use of this command. (NOTE: VMAX and VMIN are the maximum and minimum value of the problem variable associated with the grid, and are typed-out for you by VPIX 3D.)

NOCON Turn off the ccntour plotting option. (It is initially turned off.)

\section{Shading Options}

VPIX 3D produces two types of smooth-shaded half-tone pictures. In the first type, shading is done according to a light source located at infinity behind the user (i.e., a planar source). In the other, shading is done much like the contour plotting: i.e., the light intensity is assigned according to the variable value. Those grid points associated with the highest values receive the greatest intensities (appear brightest). 

Command
Action
LITE
Do shading according to a light source. (This is the initial mode.)
VARS
Do shading according to the associated problem variable.

\section{Exploded Views}

Added versatility is obtained by permitting the object to be broken down into smaller components. These exploded views can add to the underslanding of complex geometries by focusing attention on a specific area. Increased visibility can be attained by changing the scale in a given direction compared to the other two directions. Also, it is possible to remove the grid lines and show only the outside profile of the object. This is useful where contour plots are to be superimposed on the surfaces of the object. Examples appear in Fig. F-5.

\section{Selecting the Output Medium}

VPIX 3D will output its pictures io a variety of media. The user must specify the output mediurn each time in order to produce a picture.

Command

TV $n$

(TV)

DD80

DDE0A

GREY or

G32

T7Y

Command

NEXT

RSND

END

\section{Action}

Sends picture to TMDS monitor $n$. The picture will appear in the form of a line drawing. Once you get a monitor, just type TV, or the program gets confused.

Sends tine picture to dd80 file. The file is kept by the user and has the rame, VPICa, where " $a$ " is some artitrary letter. This file can be "given away" using ALLOUT. The first call to this will cause the program to request box and ID information.

Does nothing now, since the dd80A is not on-line. Will produce hardcopy of greyscale and color pictures.

Sends a shaded picture to a disk file (program asks for name). GREY produces shaded pictures with 8 levels of intensity; G32 gives 32 levels. The pictures can subsequently be viewed on the grey-level TMDS monitor by using Archuleta's GREY controllee. ${ }^{13}$

Sends picture to your teletypewriter. The picture will be a block of numbers showing the shading with " 0 " being the background intensity, and " 9 " being the highest intensity. This will give the user a rough idea of the shading.

\section{Other Commands}

\section{Action}

Closes the old data file and asks the user to specify a new one.

Cause the picture to be resent to the most recently specified medium without reprocessing picture. For use after TMDS error.

Disengages program control and gaes away nicely. 


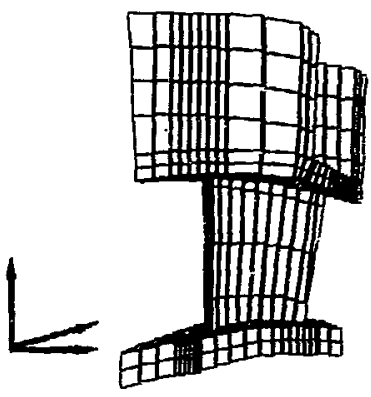

(a)
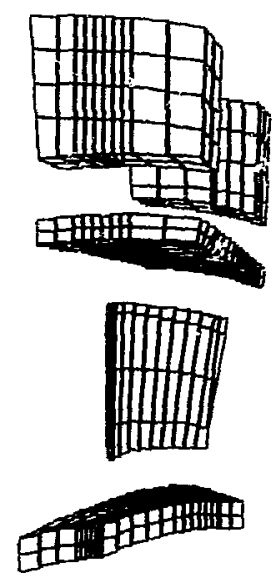

(b)
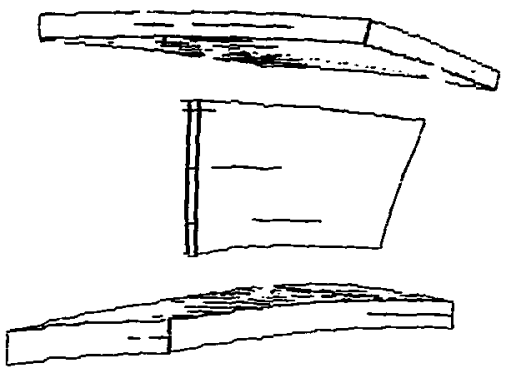

(d)

Fig. F-5. Exploded views of a gas turbine stator blade. (a) Original object. (b) Exploded view. (c) Exploded view with two narts deleted from the display, and scaling $(2 X)$ in the $x$ and $z$ directions. (d) Same as (c) but with grid lines removed.

\section{FUTURE PLARS}

A number of features will be inciuded in VPIX 3D in the future. These include the possibilities for generating color pictures on the dd80A and also for generating movies. The former will come when the dd80A comes on-line and the latter when need arises. 


\section{RUNNING THE PROGRAM}

To run VPIX 3D you nced the controllee, and a data file. To initialize, type:

$$
\text { VPIX3D / iv or VPIX3D filename } / \mathrm{t} v
$$

The filename is the name of the input data file. If one is nat specified on the execute line, the program will ask for one. If the grid has a problem variable associated with it, the first message will be

$$
\text { VMAX }=n \quad \text { VMIN }=n
$$

After that, whenever the program is ready to receive commands, it will type the prompt "OK." Commands are blank delimited and may be stacked (e.g., P 90 Y 180 TV 68 DD80). Numbers may be input as integers or as foating points. If you misspell any command, leave out a blank, or omit a numeric argument that should be there; vou will get an error message, and any commands left in the stack will be ignored. 


\section{References}

1. M. L. Wilkins, Calculations of Elsstic Plastic Flow. Lawrence Livermore Laboratory, Rept. UCRL-7322, REv. I (1969).

2. E. L. Wilson, SOLID SAP, A Static Analysis Program for Three Dimensional Solid Structures, Structural Engineering Laboratory, University of California Berkeley, Rept. UC-SESM $71-19$ (1972).

3. M. L. Wilkins, S. J. French, and M. Sorem. Proc. Intern. Conf. Numerical Mi thods Fluid Dynamics, 2nd, Berkeley, California, 1970 (Springar-Verlag. Berlin-Heidelberg, New York. 1! 71) p. 30.

4. J. von Neumann and R. D. Richtmyer. J. Appl. Phys. 21, 232 (1950).

5. O. C. Zienkiewisz. The Finite Element Method in Engineering Science (McGraw-Hill, London, 1971).

6. D. Giroux. HEMP Users Minual, Lawrence Livermore Laboratory Rept. UCRL-51079, Rev. 1 (1973).

7. B. A. Boley, and J. H. Weiner, Theory of Thermal Stresses (John Wiley and Sons Inc., New York, 1960) p. 244.

8. D. N. Hutula and S. M. Zeiler, MESH 3D: A Three-Dimensional Finite Element Mesh Generator Program for Eight-Node lsoparametric Elements, Westinghouse Elestric Corp., Atonic Power Div. Pittsburgh, Rept. WAPD-TM-1079, (1972).

9. M. Archuleta, Hidden Surface Processing, Lawrence Livermore Laboratory, Rept. UCID-30057 (1973).

10. M. Archuleta, Hidden Surface Line-Drawing Algorithm, Computer Science Department, University of Utah, Rept. UTECH-CSc-72-12! (1972).

11. H. Gouraud, Computer Display of Curved Surfaces, University of Utah, Rept. UTECH-CSc-71-113 (1971).

12. G. S. Watkins, A Real-Time Visible Surface Algorithm, Computer Science Department, University of Utah, Rept. UTECH-CSc-70-101, (1970)

13. M. Archuleta, Luwrence Livermore Laboratory. private communication (Jan., 1974). 